\title{
Gearbox Modeling and Load Simulation of a Baseline 750-kW Wind Turbine Using State-of-the- Art Simulation Codes
}

F. Oyague

Technical Report NREL/TP-500-41160

February 2009 


\section{Gearbox Modeling and Load}

Simulation of a Baseline 750-kW Wind Turbine Using State-of-theArt Simulation Codes

\section{F. Oyague}

Prepared under Task No. WER8.2001

National Renewable Energy Laboratory 1617 Cole Boulevard, Golden, Colorado 80401-3393 303-275-3000 • www.nrel.gov

NREL is a national laboratory of the U.S. Department of Energy

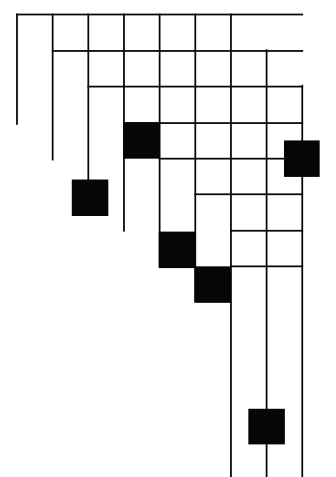




\section{NOTICE}

This report was prepared as an account of work sponsored by an agency of the United States government. Neither the United States government nor any agency thereof, nor any of their employees, makes any warranty, express or implied, or assumes any legal liability or responsibility for the accuracy, completeness, or usefulness of any information, apparatus, product, or process disclosed, or represents that its use would not infringe privately owned rights. Reference herein to any specific commercial product, process, or service by trade name, trademark, manufacturer, or otherwise does not necessarily constitute or imply its endorsement, recommendation, or favoring by the United States government or any agency thereof. The views and opinions of authors expressed herein do not necessarily state or reflect those of the United States government or any agency thereof.

Available electronically at http://www.osti.gov/bridge

Available for a processing fee to U.S. Department of Energy and its contractors, in paper, from:

U.S. Department of Energy

Office of Scientific and Technical Information

P.O. Box 62

Oak Ridge, TN 37831-0062

phone: 865.576 .8401

fax: 865.576 .5728

email: mailto:reports@adonis.osti.gov

Available for sale to the public, in paper, from:

U.S. Department of Commerce

National Technical Information Service

5285 Port Royal Road

Springfield, VA 22161

phone: 800.553 .6847

fax: 703.605.6900

email: orders@ntis.fedworld.gov

online ordering: http://www.ntis.gov/ordering.htm 


\section{Executive Summary}

The wind energy industry continually evolves, and industry professionals have streamlined gearbox design to a consensus configuration. This configuration and its design iteration have existed for many years; consequently, design and manufacturing flaws have been minimized sequentially. Regardless of the maturity of the gearbox design and design process, however, most wind turbine downtime is attributed to gearbox-related issues. Moreover, gearbox replacement and lubrication accounts for $38 \%$ of the parts cost of the entire turbine.

Several hypotheses have been offered to explain gearbox failure, including the absence of a number of load cases relevant to the design process; the transfer of nontorsional loads between the different components of the drivetrain; the lack of a uniform standardization of bearing-life analysis calculations; and poor communication between wind turbine designers, gearbox suppliers, and bearing providers.

This report discusses determining a method for revealing the missing loading conditions that should be factored into the gearbox-design process. This objective is achieved by development of a number of analytical models that sequentially increase in complexity, and which are capable of reproducing the dynamical behavior of the internal components of the drivetrain. Additionally, the parameters obtained from these models are correlated with the gearbox-design process. Importantly, the models developed are offered freely to improve communication and to open information-sharing avenues between manufacturers and designers involved in the non-vertical design process.

The models reveal that the level of complexity does not greatly affect torsional behavior. Furthermore, models of higher complexity are capable of providing important insight into the loading conditions for the bearings of the gearbox, and still account for loads generated by geartooth interactions. 


\section{Acknowledgements}

I would like to thank my primary supervisor Professor Dr. Dipl.-Ing. Martin Kühn, my secondary supervisor Dipl.-Ing. Stefan Hauptmann, and my COMMAS supervisor Professor Dr.-Ing. Bernd Kröplin, for arranging the integration of the COMMAS Masters Program with the Endowed Chair of Wind Energy (SWE), and thus allowing me the opportunity to participate in cuttingedge research.

Thanks also go to Chief Engineer Sandy Butterfield, my external supervisor, who allowed me the opportunity to research and write this report at the National Renewable Energy Laboratory (NREL), and who provided constant advice and guidance; and to Ed Hahlbeck, Don McVittie, and Brian McNiff, who shared their expertise in the gearbox-design process and the wind turbine industry, and gave me valuable advice.

I thank INTEC GmbH for providing the multibody system code SIMPACK, which made possible the development of the progressive models used in this report.

Last, but not least, I thank my family and friends for their ever-constant support and guidance. Specifically, I would like to acknowledge Maria Christina and Marnix Vanderplas, who generously provided the financial support that enabled me to produce this report. 


\section{Table of Contents}

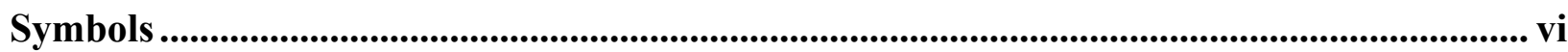

List of Figures................................................................................................................................... vii

Introduction ............................................................................................................................. 1

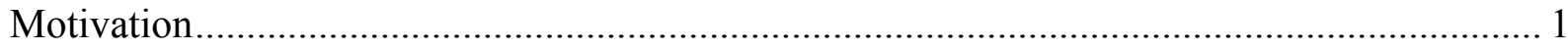

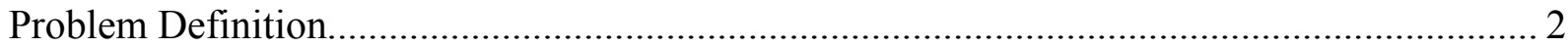

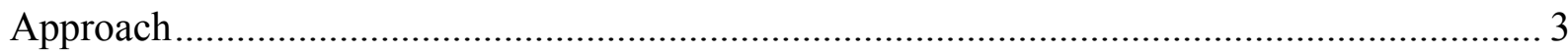

Wind Turbine Configurations .................................................................................................................. 4

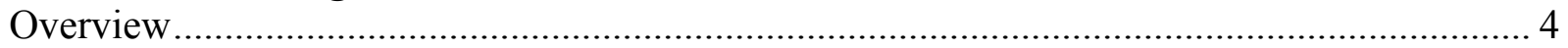

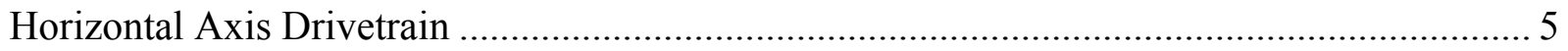

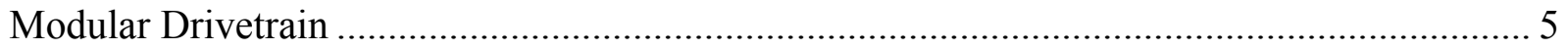

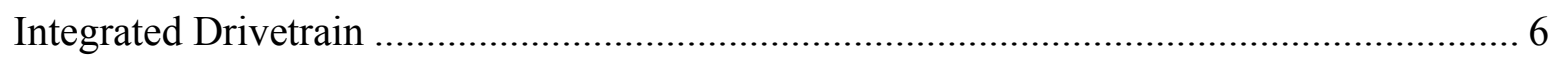

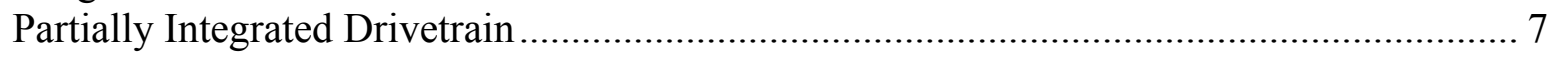

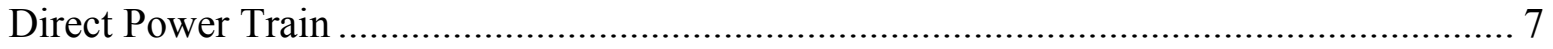

Drivetrain Configuration Comparison ............................................................................. 8

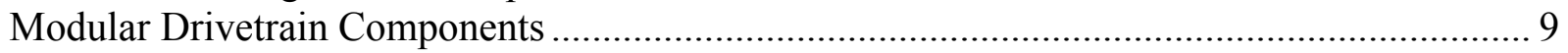

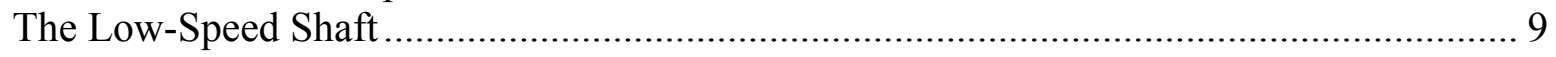

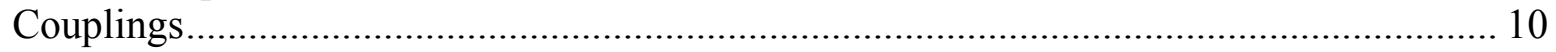

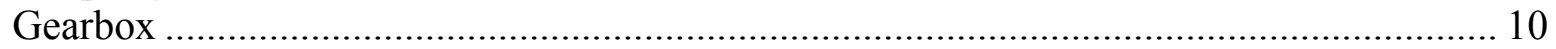

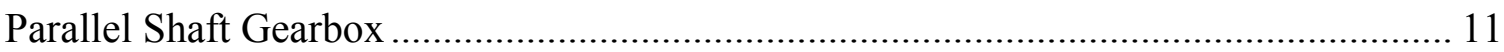

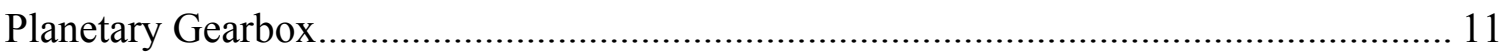

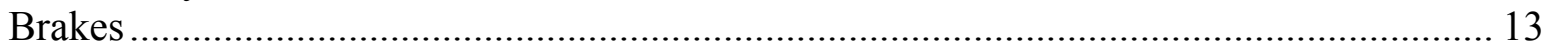

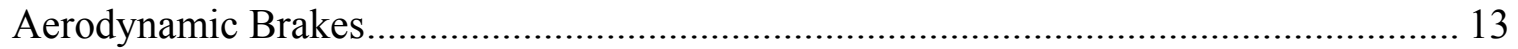

Mechanical Brakes............................................................................................ 14

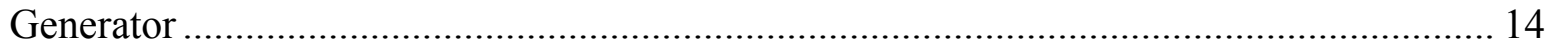

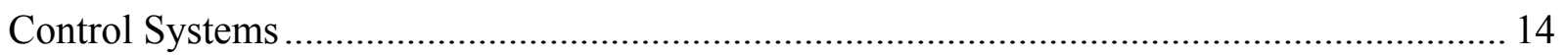

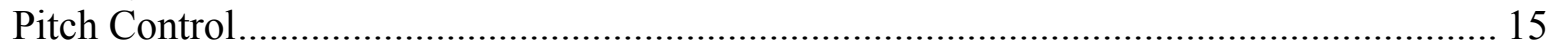

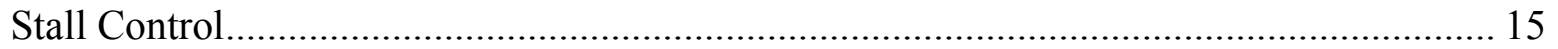

Pitch Control Versus Stall Control ............................................................................. 15

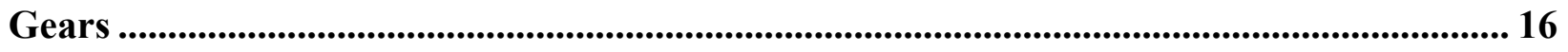

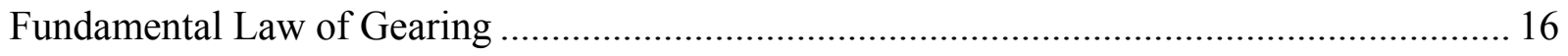

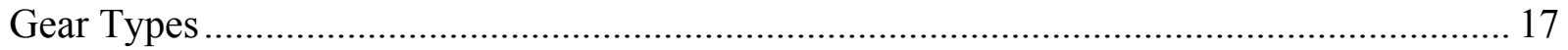

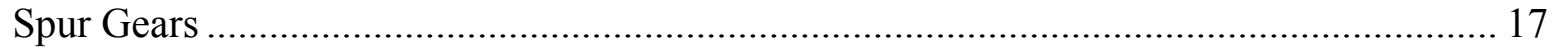

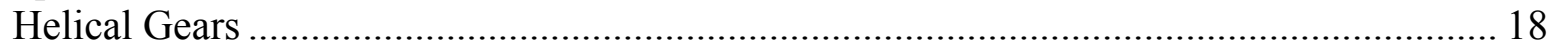

Involute Gear Tooth Nomenclature ............................................................................. 18

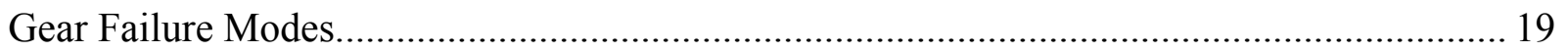

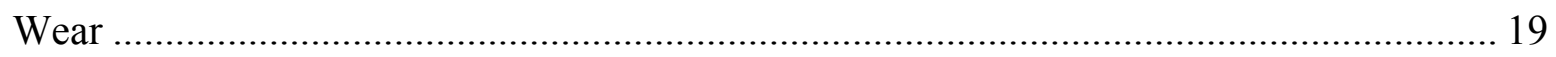

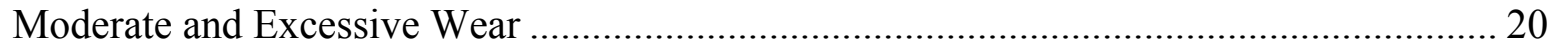

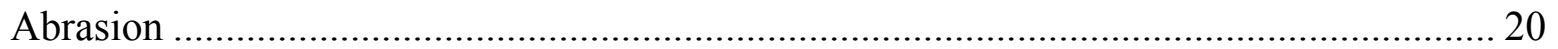

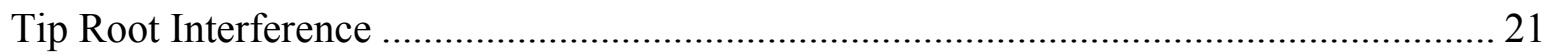

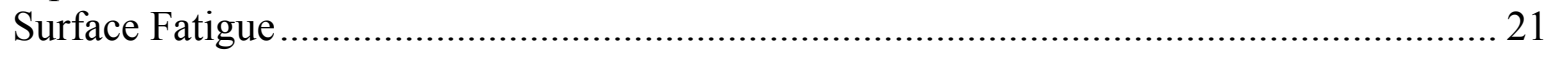

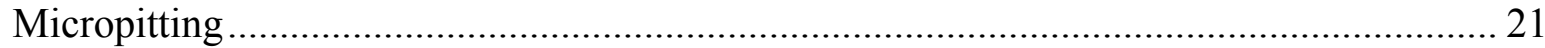

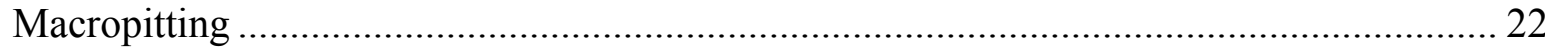

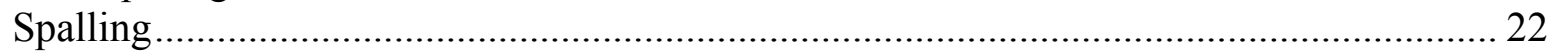

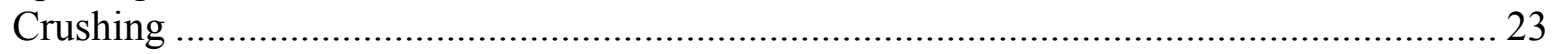




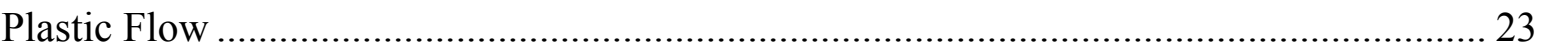

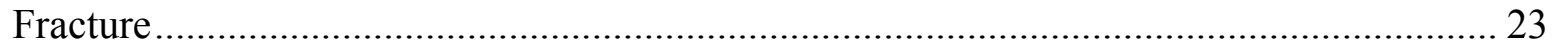

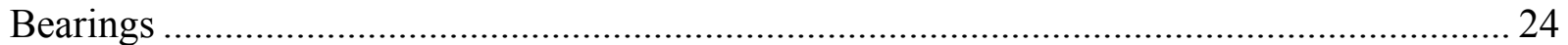

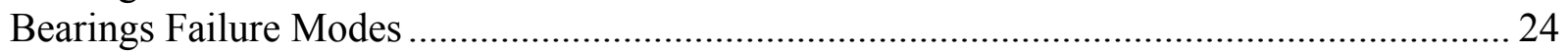

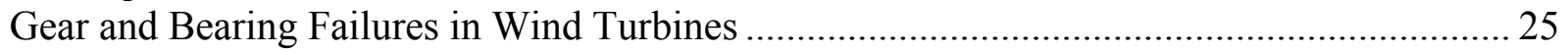

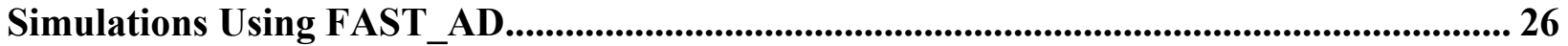

Multibody System Simulations ..................................................................................................... 27

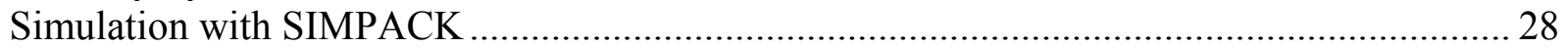

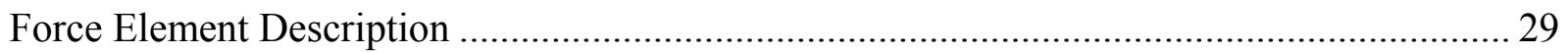

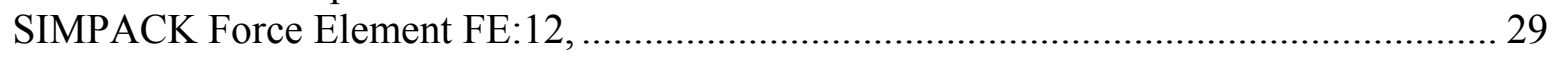

Torsion-Spring Suspension (Force Law Based on the Joint State Quantities)...................... 29

SIMPACK Force Element FE:14, Gearbox with Elastic Transmission ................................. 29

SIMPACK Force Element FE:225, Component Force Element ............................................ 29

Simulation Theoretical Input Parameters .....................................................................30

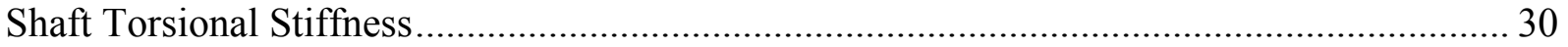

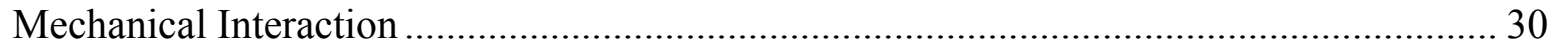

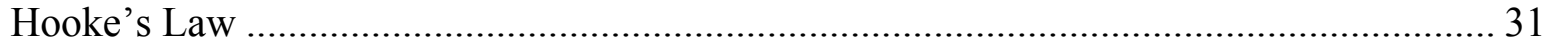

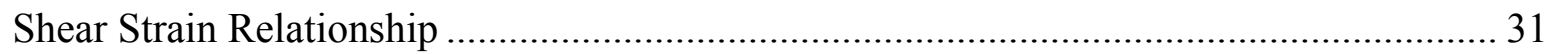

Torsional Deflection of a Circular Shaft .................................................................... 32

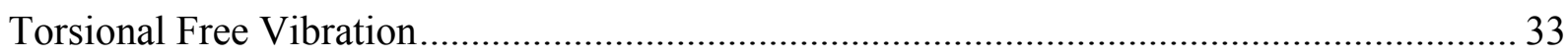

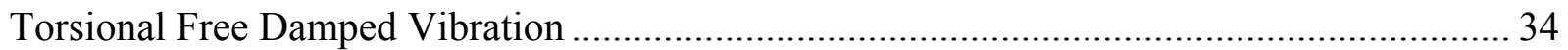

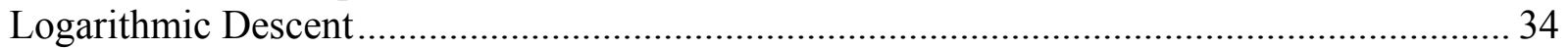

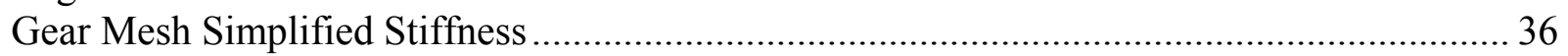

Mesh Stiffness Calculation Input Parameters …………........................................................... 37

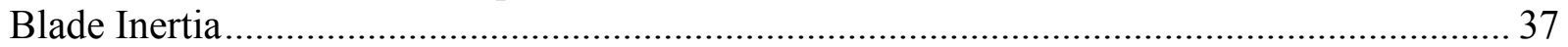

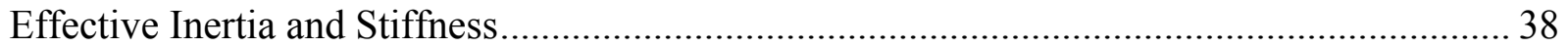

Progressive Stage Description.........................................................................................40

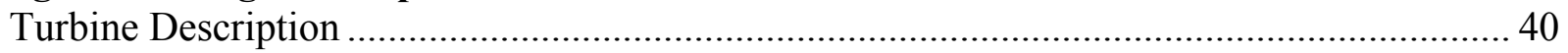

Stage 1. Simplified Complete Drivetrain Model ..................................................................... 40

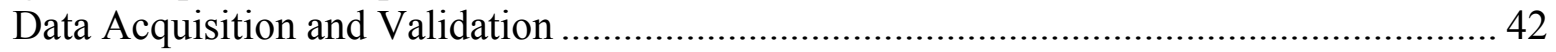

Stage 2. Simplified Rotor and Generator with Multiple-Stage Gearboxes............................... 47

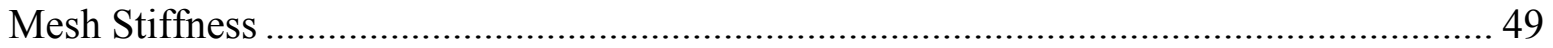

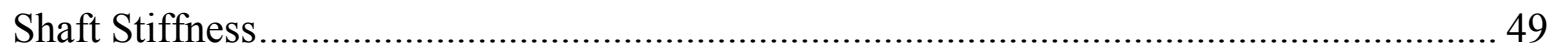

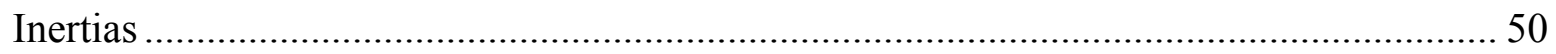

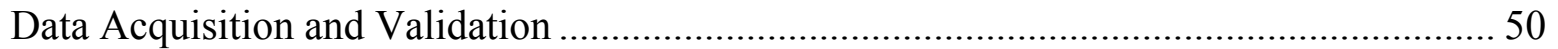

Stage 3. Multiple-Stage Gearboxes with Contact Element Implementation .............................51

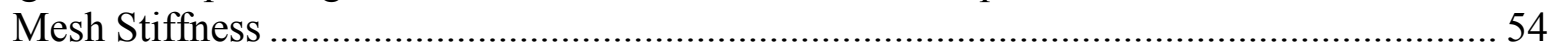

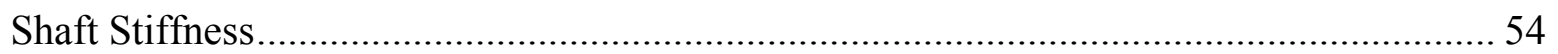

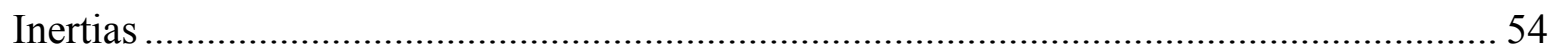

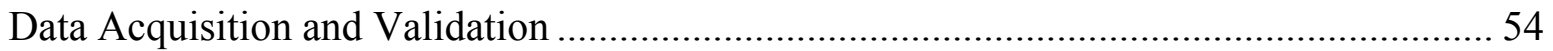

Stage 4. Multiple-Stage Gearboxes with Contact Element Implementation and Bearing

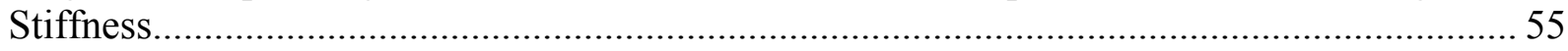

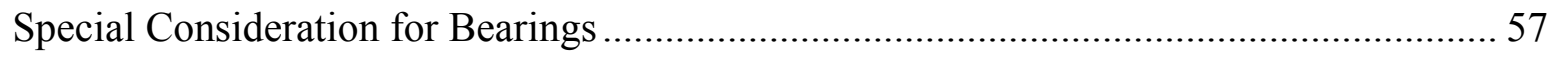

Validation and Parameter Acquisition................................................................................ 57 
FAST Model Description...........................................................................................................5 58

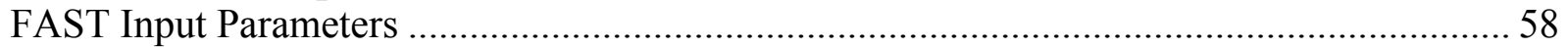

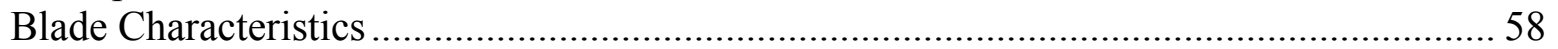

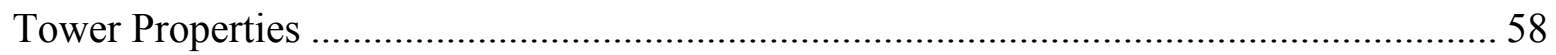

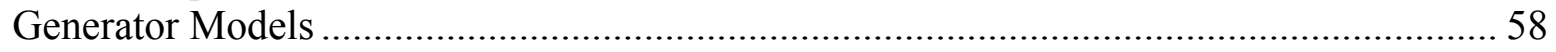

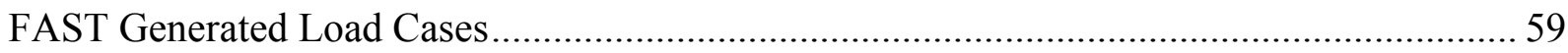

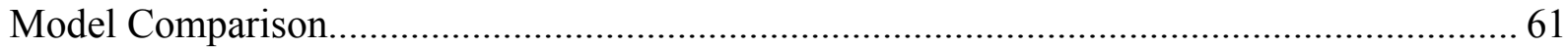

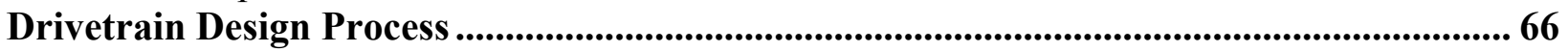

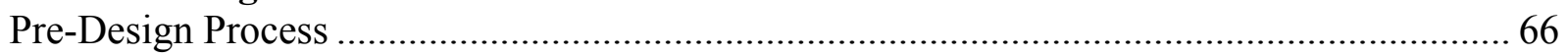

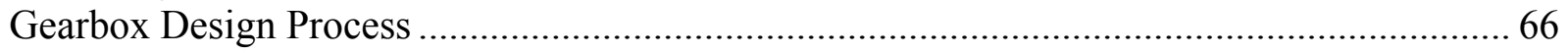

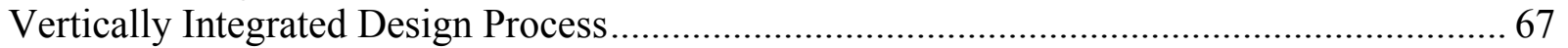

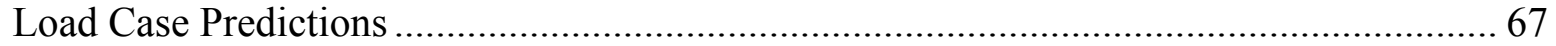

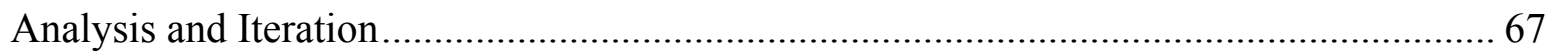

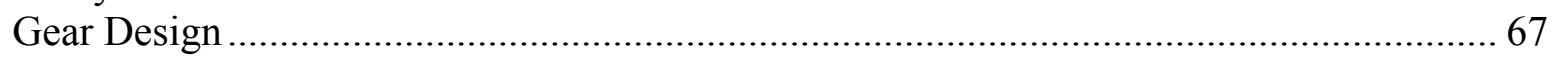

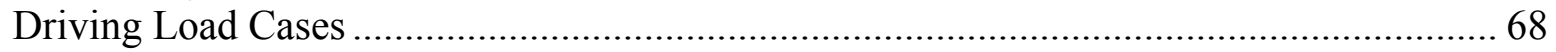

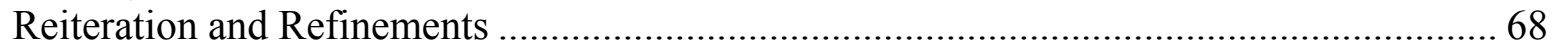

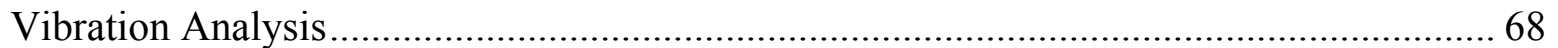

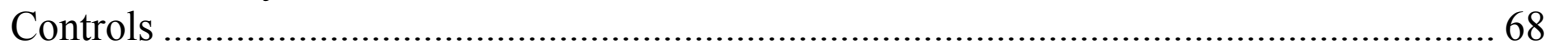

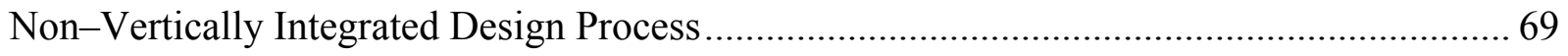

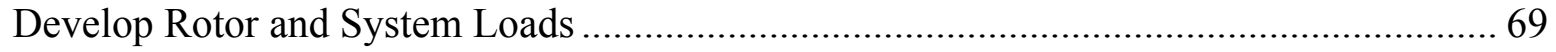

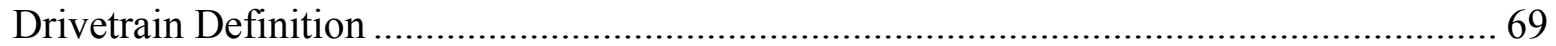

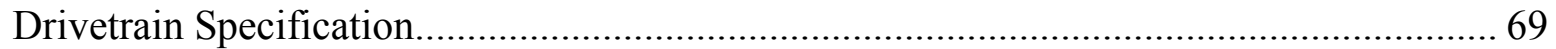

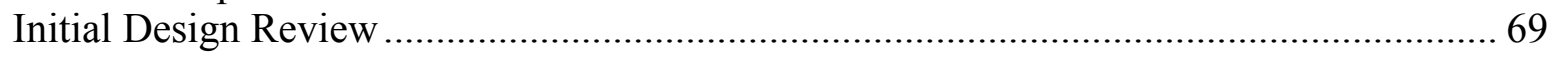

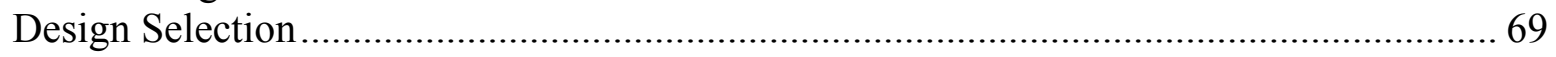

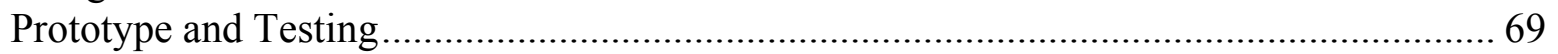

Comparison of Drivetrain Design Processes .................................................................. 70

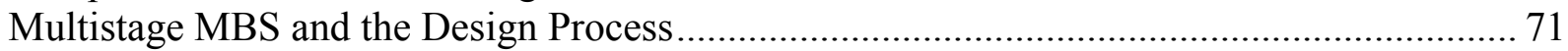

Conclusions and Final Remarks.......................................................................................... 72

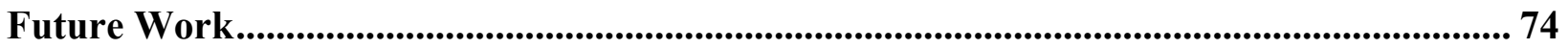

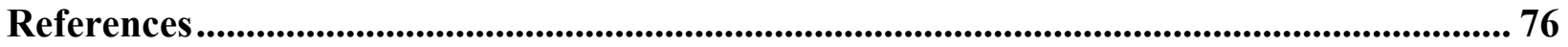

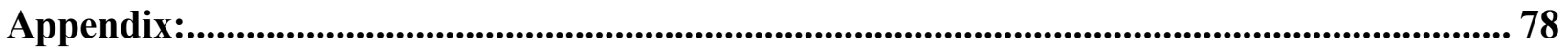

Aerodynamic Simulation.............................................................................................................. 78

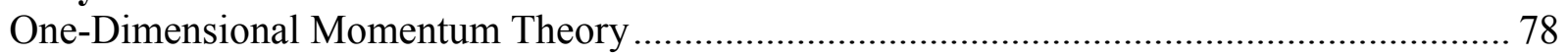

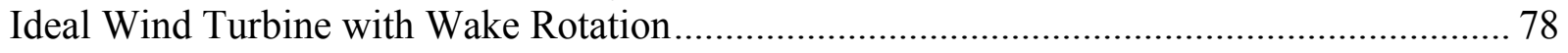

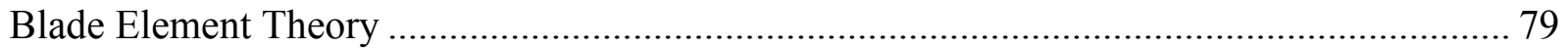




\section{Symbols}

b

$c$

$c$

$C_{l}$

$C_{d}$

D

dA

$D_{\text {ring }}$

$D_{\text {Sun }}$

E

G

h

$I_{a}$

$I_{m}, J$

$I_{p}$

$k$

K

$k_{\text {eq }}$

$k_{t}$

l

L

$l_{s}$

$m$

$\dot{m}$

ma

$m_{G}$

$m v$

$n_{H s s}$

$n_{\text {LSS }}$

$P$

$\omega_{\text {in }}$

$\omega_{\text {out }}$

$r_{\text {in }}$

$r_{\text {out }}$

$r_{1}$

$y_{\max }$

$T_{r o t}$

$V_{1}$

$V_{3}$

$U$

$\rho$

$\sigma$

$\varepsilon$

$\gamma$
With of cross-sectional area [m]

Airfoil cord length [m]

Damping coefficient $[\mathrm{N} / \mathrm{m} / \mathrm{sec}]$

Lift coefficient [-]

Drag coefficient [-]

Drag force [N]

infinitesimal element [-]

Ring gear diameter $[\mathrm{m}]$

Sun gear diameter $[\mathrm{m}]$

Young's modulus $\left[\mathrm{N} / \mathrm{m}^{2}\right]$

Shear modulus of elasticity $\left[\mathrm{N} / \mathrm{m}^{2}\right]$

Height of the cross-sectional [m]

Area moment inertia $\left[\mathrm{m}^{4}\right]$

Mass moment of inertia $\left[\mathrm{kg} \mathrm{m}^{2}\right]$

Polar moment of inertia $\left[\mathrm{m}^{4}\right]$

Spring constant $[\mathrm{Nm} / \mathrm{rad}]$

Stiffness [Nm/rad]

Equivalent stiffness [Nm/rad]

Torsional stiffness $[\mathrm{Nm} / \mathrm{rad}]$

Length of rod under torsion [m]

Lift force $[\mathrm{N}]$

Airfoil span [m]

Mass [kg]

Mass flow rate $[\mathrm{kg} / \mathrm{sec}]$

Mechanical advantage [-]

Gear ratio [-]

Velocity ratio [-]

High-speed shaft angular velocity [-]

Low-speed shaft angular velocity [-]

Period of oscillation [sec]

Angular velocity input [rad/sec]

Angular velocity output [rad/sec]

Pitch radius of input gear [m]

Pitch radius of output gear [m]

Radius of rod under torsion [m]

Maximum beam deflection [m]

Rotor thrust [N]

Incoming wind velocity $[\mathrm{m} / \mathrm{s}]$

Outgoing wind velocity $[\mathrm{m} / \mathrm{s}]$

Undisturbed fluid velocity $[\mathrm{m} / \mathrm{sec}]$

Density $\left[\mathrm{kg} / \mathrm{m}^{3}\right]$

Stress $\left[\mathrm{N} / \mathrm{m}^{2}\right]$

Strain [-]

Shear strain deformation [rad] 
Shear stress $\left[\mathrm{N} / \mathrm{m}^{2}\right]$

Angle of torsional deflection [rad]

Applied torque [N/m]

Undamped natural frequency $[\mathrm{rad} / \mathrm{sec}]$

Undamped natural frequency [HZ]

Acceleration $\left[\mathrm{m} / \mathrm{sec}^{2}\right]$

Velocity $[\mathrm{m} / \mathrm{sec}]$

Position [m]

Damping ratio [-]

Logarithmic descent [-]

Rotation around the $\mathrm{X}$ axis [-] 


\section{List of Figures}

Figure 1. Downtime hours accumulated from 2003 to 2007 for wind turbines operating in

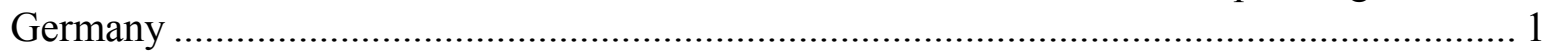

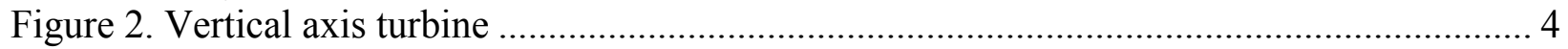

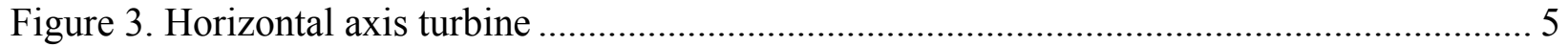

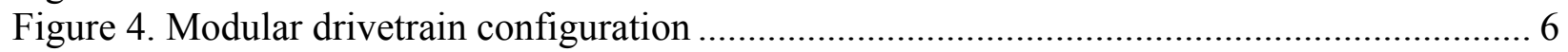

Figure 5. Integrated drivetrain from Wind World W-2700 ............................................ 7

Figure 6. Partially integrated drivetrain .......................................................................... 7

Figure 7. Enercon direct power train ........................................................................... 8

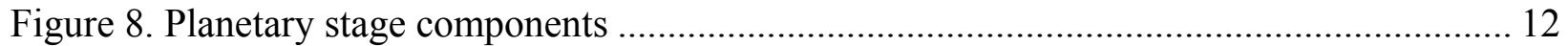

Figure 9. Planetary stage components rotational direction .............................................. 13

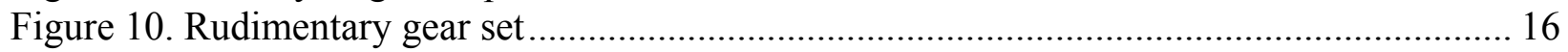

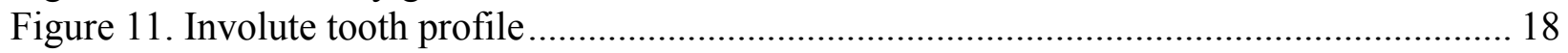

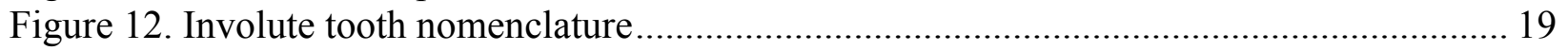

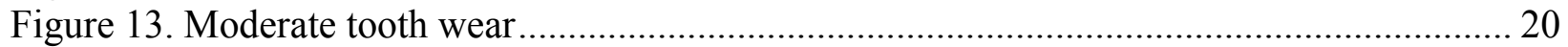

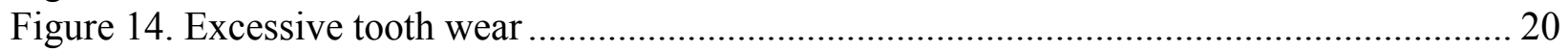

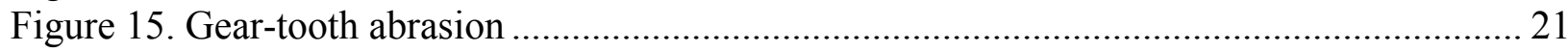

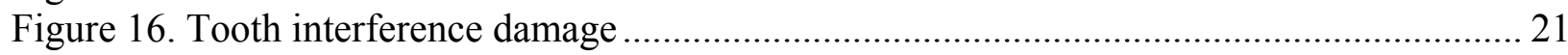

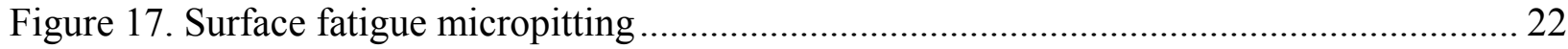

Figure 18. Surface fatigue macropitting ................................................................... 22

Figure 19. Surface fatigue spalling .............................................................................. 23

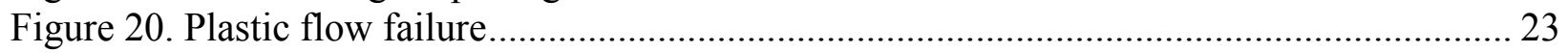

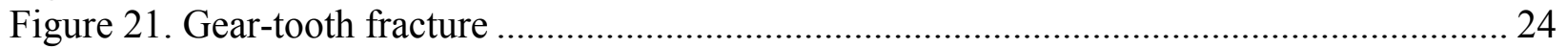

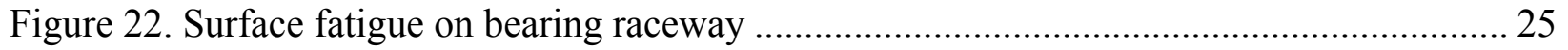

Figure 23. Adhesive wear produced by overheating ..................................................... 25

Figure 24. Absolute and relative coordinates ................................................................... 28

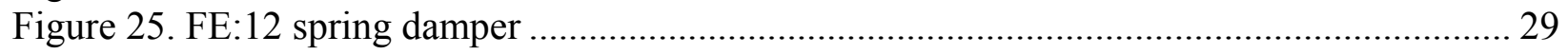

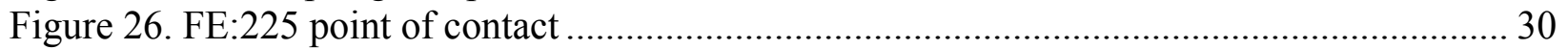

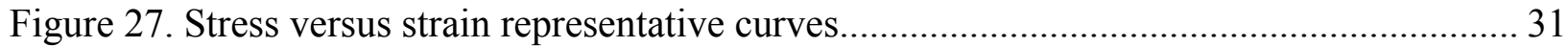

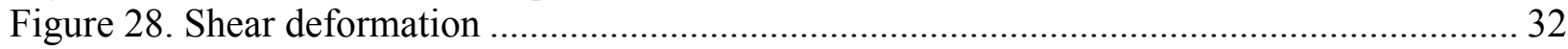

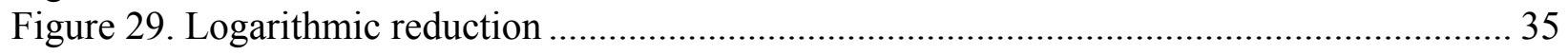

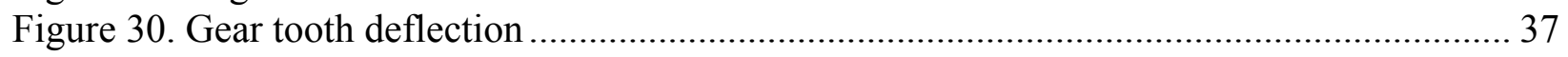

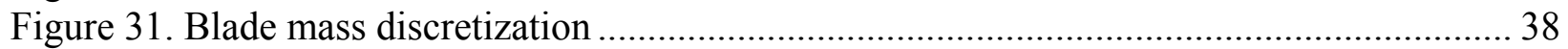

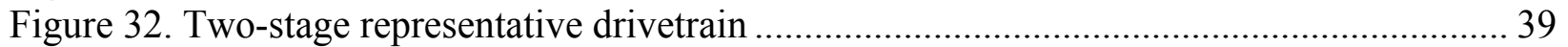

Figure 33. Equivalent one-stage drivetrain with single stiffness and inertia........................... 39

Figure 34. Graphic representation of equivalent stiffness ............................................. 40

Figure 35. Stage 1 graphical representation........................................................................ 41

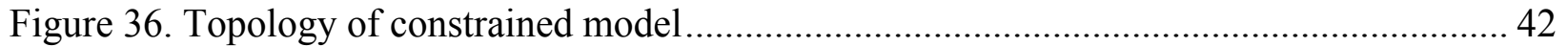

Figure 37. Topology of unconstrained model...................................................................... 42

Figure 38. Experimental data and secondary frequency response .......................................... 44

Figure 39. True response (left) and collected response (right) ........................................... 45

Figure 40. Experimental data and simulated response....................................................... 46

Figure 41. Stage 1 simulated response with refined integration time step .............................. 46

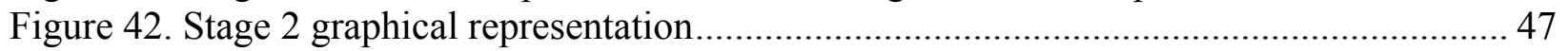

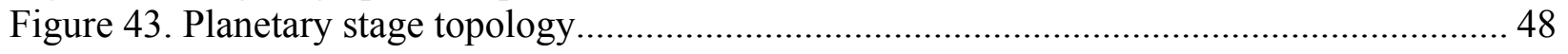


Figure 44. Subsequent gearbox stages topology .............................................................. 49

Figure 45. Stage 2 model simulated response with fine integration time step ......................... 51

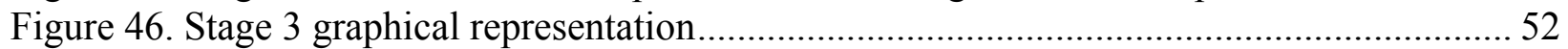

Figure 47. Topology of planetary stage with FE:225 ...................................................... 53

Figure 48. Topology of subsequent gearbox stages with FE:225 .................................... 53

Figure 49. Stage 3 model simulated response with fine integration time step .......................... 55

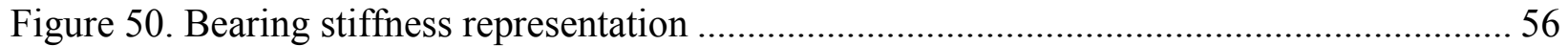

Figure 51. Planetary stage with added degrees of freedoms and force constraints ................... 57

Figure 52. FAST_AD generator models ....................................................................... 59

Figure 53. FAST_AD simulated braking maneuver and event description............................... 59

Figure 54. Planet carrier displacement under braking event............................................... 60

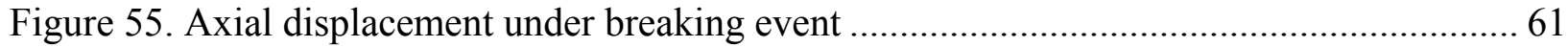

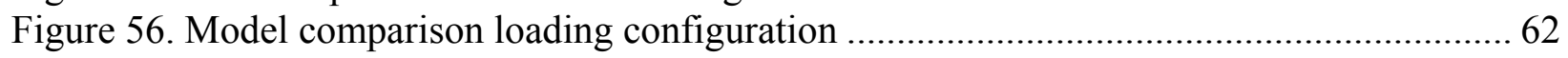

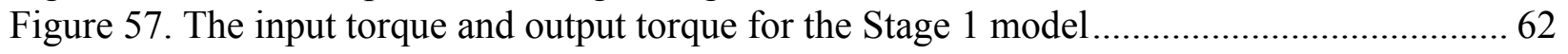

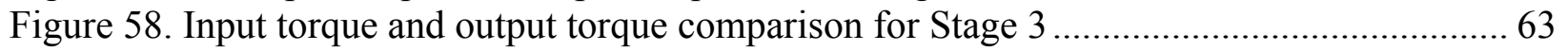

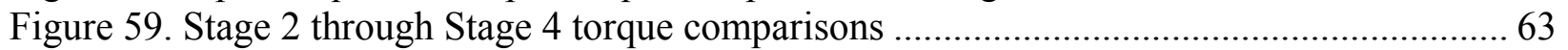

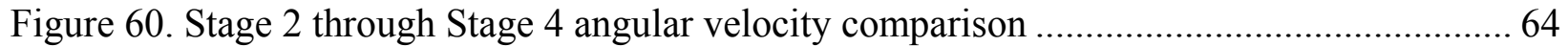

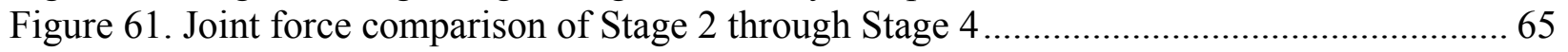

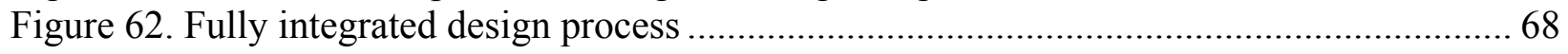

Figure 63 . Non-vertically integrated design process..................................................... 70

Figure 64. Stiffness coefficient decay from experimental data ............................................. 74

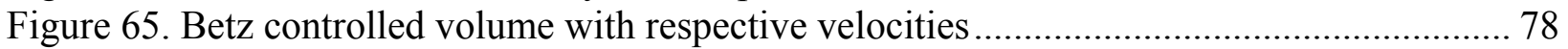

Figure 66. Controlled volume with rotational wake ......................................................... 79

Figure 67. Curves for coefficient of lift and coefficient of drag .......................................... 80 


\section{Introduction}

The growing consciousness of global climate change has helped the importance of renewable energy become paramount. The wind industry, in particular, has seen an unquenchable demand as a result, and the need for reliable and affordable wind turbines now is all the more apparent. Unfortunately, recurrent drivetrain failures have characterized the industry and have prevented the turbines from achieving their intended 20-year design life (see Figure 1).

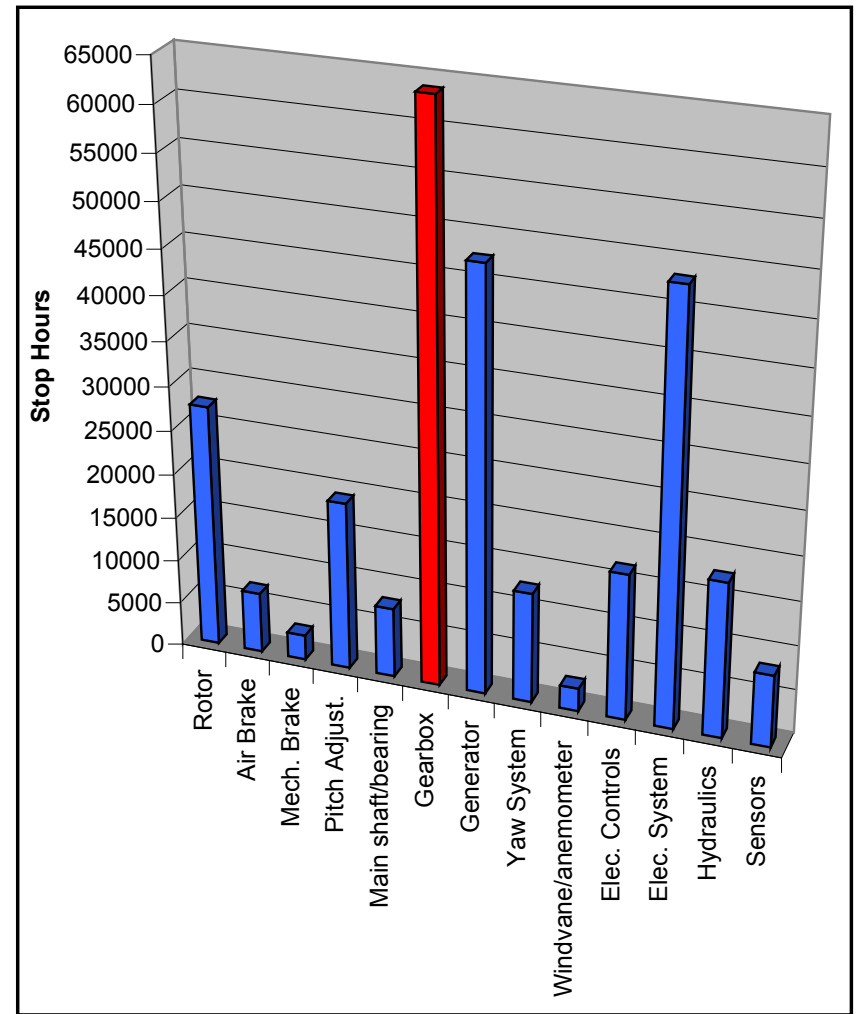

Figure 1. Downtime hours accumulated from 2003 to 2007 for wind turbines operating in Germany

The component most responsible for downtime is the gearbox [1]. Gearbox replacement and lubrication account for $38 \%$ of the parts cost for the entire turbine system [2]. This situation calls for the implementation of new and advanced simulation techniques to be integrated into the gearbox-design process so that this component can meet its intended design life.

\section{Motivation}

This report is a combination of the efforts of the National Renewable Energy Laboratory (NREL) (United States) and the Endowed Chair of Wind Energy (SWE) at the University of Stuttgart (Germany). It is part of the gearbox reliability collaborative (GRC) project, which seeks to improve gearbox reliability and, consequently, wind turbine reliability. The GRC originates from NREL's National Wind Technology Center (NWTC). The GRC attempts to bring together the various parties involved in the gearbox design process with the common goal of the improvement of the lifetime of gearboxes. It seeks to achieve this by exploring three avenues of research: drivetrain numerical analysis and modeling, full-scale dynamometer testing, and field testing. 


\section{Problem Definition}

The drivetrain has the important task of transforming the rotational energy of the rotor into electrical power. The drivetrain is composed of several elements, each of which contributes to a specific task. Except for the direct drivetrain, all drivetrains have a gearbox. The gearbox is responsible for increasing the angular velocity transmitted from the rotor to the generator, to satisfy the velocity required by the generator. It is the component of greatest mechanical complexity in the drivetrain and, as noted, is responsible for most wind turbine operational downtime and for increased costs.

Several hypotheses have been offered to explain the early failure of gearboxes. Among these is the possibility that a number of load cases are not considered in the loads document used for the design of the independent components of the drivetrain. There also is the possibility that nontorsional loads and dynamical effects are transferred among the components of the drivetrain. Lastly, it is possible that the reliability of bearings is not uniform throughout the industry. This is due to an incomplete definition of the assumptions and parameters specified in the current bearing standards that subsequently, has led individual manufactures to create in-house codes for predicting bearing life.

Another important characteristic of gearbox failure is that it occurs in turbines of several sizes having the same or similar configurations. It is believed that problems present in previous turbine models (ranging from $500 \mathrm{~kW}$ to $1000 \mathrm{~kW}$ ) have persisted, and therefore they still occur in today's larger turbines. This is very relevant, because if the problem in smaller turbines was solved then the solution could be extrapolated to larger turbines. This situation is advantageous in that working with smaller turbines reduces both the cost of use and the availability of test subjects presently operating in the field. Furthermore, this translational property between turbines enabled the use of experimental data collected from a $750-\mathrm{kW}$ turbine in the field to validate the progressive models described in this report.

Although the presence of problems involving the gearbox is evident, the characterization and reasons for these problems are not as clear. Several observations have been made with respect to gearbox failures in an attempt to better characterize the problem. For example, over the years gearboxes have been streamlined by several independent manufacturers and progressively have converged to a similar configuration. This configuration thus represents a mature consensus design of manufacturers, and it is used almost universally in the industry. Failures occur in gearboxes of this configuration independent of the manufacturer and any slight differences in the actual design. This suggests that each independent manufacturer is performing the same design routine. Additionally, because the industry is highly competitive and because it can be assumed that manufacturers are capable of correcting quality-control problems, it follows logically that the failures experienced are not caused by poor workmanship. Therefore the problems inherent to gearboxes appear to be a product of flaws in the design process.

The gearbox-design process is characterized by the integration of multiple disciplines which work independently toward the eventual integration of many different components. Each internal component that comprises the gearbox is affected by its surrounding components. Similarly, the gearbox is affected by components that are external to it and which comprise the drivetrain.

Because of these relationships, the design and manufacture of each component also is dependent on these component interactions. Thus, to appropriately follow the gearbox-design process, all 
parties involved in the process must have a complete knowledge of the behavior of all components. This necessitates, for example, that the wind turbine manufacturers understand the behavior of the gearbox, that the gearbox manufactures understand the loading conditions and interactions of the drivetrain and the gearbox, and that the bearing manufactures understand the interaction of the internal components of the gearbox. Essentially, a level of absolute transparency and comprehensive information sharing is required for the design to succeed.

Unfortunately, sharing knowledge is very difficult due to proprietary information barriers; there is competition between manufacturers and suppliers because they share common interests and clients. A single wind turbine company might have more than one gearbox supplier, for example, or a single gearbox supplier might have more than one bearing supplier. Conversely, the gearbox supplier might be working with multiple wind turbine manufacturers. It is this level of intense competition that makes transparency and information sharing virtually impossible.

\section{Approach}

This report covers a small portion of the enormously detailed analytical task of the gearbox reliability collaborative. Its approach seeks to integrate into the drivetrain-design process several numerical models which capture the dynamical nature of the drivetrain. These dynamic models progressively build in complexity, and aim to provide insight into the internal forces inherent to the dynamical behavior of the drivetrain. Additionally, the progressive nature of these models offers a method of filtration for sensitive information shared among the different parties of the design process - ultimately increasing the transparency of the design process.

Due to the reciprocal characteristics of the interactions among the dynamical components of the wind turbine, a fully coupled model which integrates all of these components has proven valuable [32]. The scope of this report is limited to the drivetrain-more specifically to the gearbox. The limited scope required the integration of different simulation codes to more closely mimic the behavior of a fully coupled model. These codes included aeroelastic codes used to create loading conditions for the gearbox models (described below).

The implementation of aeroelastic software has been extremely popular due to its ability to capture the interactions between inertial, elastic, and aerodynamic forces. This type of simulation can predict the general behavior of the wind turbine and its interactions with the wind. In this type of simulation the drivetrain typically is extremely simple. It provides a good approximation for the overall behavior of the entire turbine, although it neglects the interaction of the drivetrain's internal components. Due to the proven capabilities of aeroelastic software to closely represent wind turbine systems, this software was used in combination with a multibody system software.

The multibody system simulation approach was chosen to generate the models due to its ability to represent the drivetrain in a more detailed manner. This level of detail reveals important information of the internal loading conditions resulting from the dynamical interactions. This approach represents each component of the drivetrain as a rigid body, and defines its interaction with the other components. The representation of each component as a rigid body reduces the number of degrees of freedom (DOF), which enables the simulation to be fast and computationally light. 
The integration of these two powerful tools resulted in progressive comprehensive models, capable of generating load cases for the individual components of the drivetrain. Ideally, these load cases should be integrated into the standard gearbox-design process, because typically the process does not fully account for the dynamical behavior of the individual components of the drivetrain. Additionally, the models were compared to each other-from least complex to most complex - to identify the level of detail and fidelity that each model could generate. This information can be used by a gearbox designer to select the drivetrain model complexity that is most appropriate for an individual task.

\section{Wind Turbine Configurations}

\section{Overview}

This section describes the basic configuration of the most common wind turbines present in the industry today. It emphasizes the components of the horizontal axis turbine; more specifically, the modular drivetrain configuration is emphasized and each of its components is described. This section is very important, because the described components must be simulated. Understanding their behaviors and their interactions with the other components therefore is imperative.

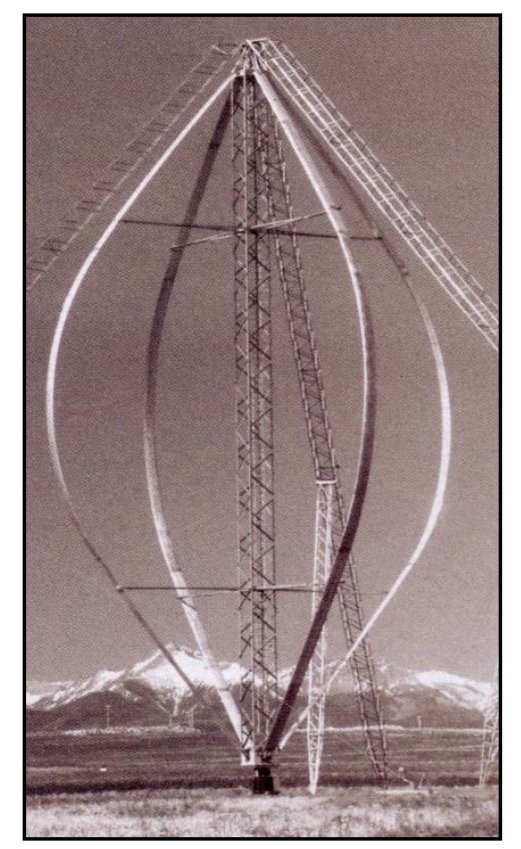

Figure 2. Vertical axis turbine [25]

Two primary structural classifications of wind turbines commonly are used: the vertical axis wind turbine and horizontal axis wind turbine. The advantage of vertical axis turbines (Figure 2) is that they are omni-directional - they have the ability to accept wind from every direction. This eliminates the problem of orienting the rotor with respect to the wind. Vertical axis turbines also have drivetrains that do not meet their expected design life. Independent of the advantages of the vertical axis configuration, horizontal axis turbines (Figure 3) have proven to be more efficient. Not surprisingly the horizontal axis design currently is the most popular design in the industry and is the design upon which this report focuses. [25] 


\section{Horizontal Axis Drivetrain}

As noted, the drivetrain is the component of the wind turbine that transforms the mechanical energy generated by the rotor into electrical energy. There are many possible configurations for the power train depending upon the designer criteria. Four common configurations include the modular drive, the integrated drivetrain, the partially integrated drivetrain, and the direct drivetrain. There currently is no common consensus with respect to which configuration is most advantageous.

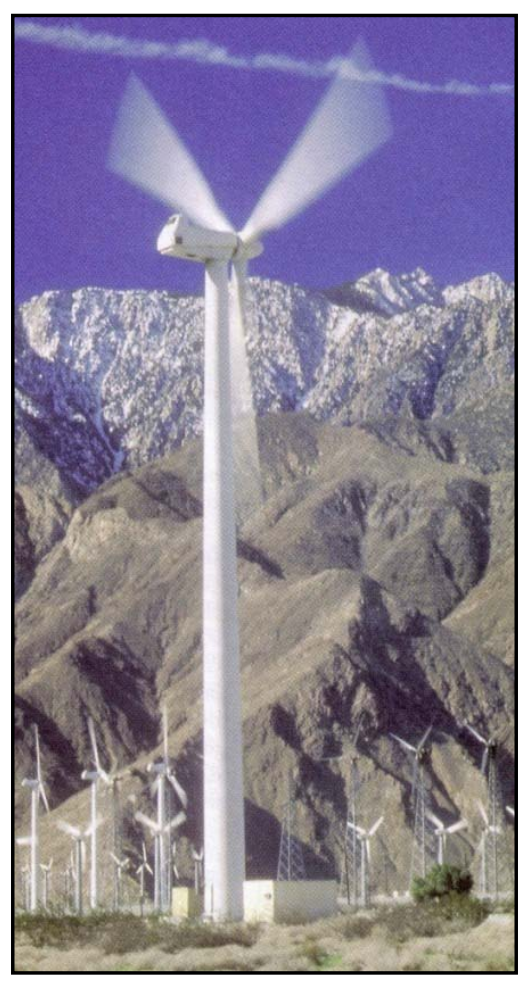

Figure 3. Horizontal axis turbine [25]

\section{Modular Drivetrain}

Currently, most operating turbines follow the modular configuration. All individual components of the drivetrain are mounted onto the bedplate, and the bedplate is designed to be torsionally stiff. Nevertheless, there is debate surrounding the bedplate's actual behavior that suggests that it is not as stiff as it should be, and that its flexibilities influence not only the interaction between the different components of the drivetrain but also its vibrational behavior [28]. The main components of the drivetrain are the rotor shaft or low-speed shaft, the gearbox, the brakes, and the generator.

The modular configuration allows a non-vertical design process, which means that different suppliers can contribute to the development of the different components of the drivetrain. This inherently reduces the overall cost by fomenting a competitive environment among suppliers, and reduces the in-house requirements of the turbine manufacturer. Figure 4 shows the typical configuration for a modular drivetrain [3]. 


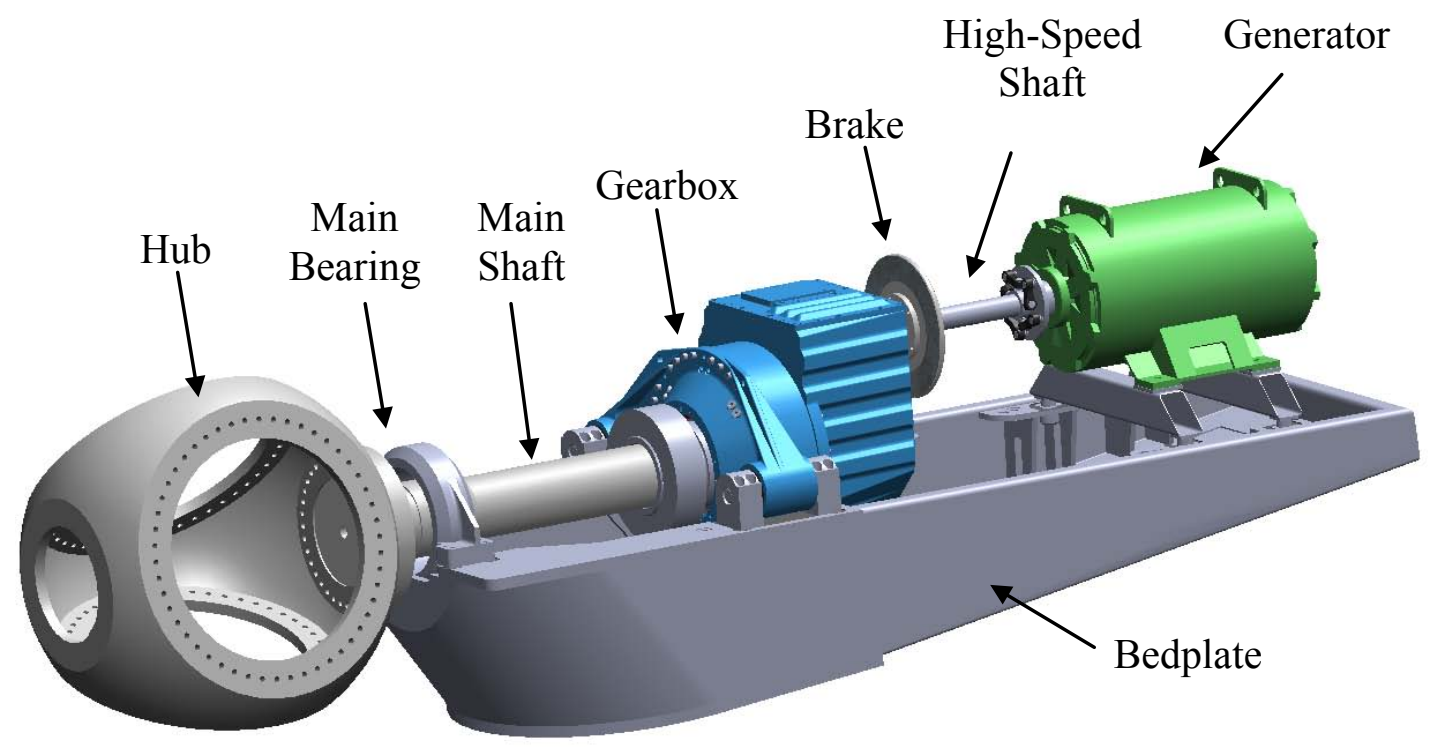

Figure 4. Modular drivetrain configuration

\section{Integrated Drivetrain}

As its name indicates, the integrated drivetrain includes the components of the modular type discussed above. Generally, the gearbox becomes the main component of the system and the remaining components are attached or flanged to it. This compact design is not dependent on the bedplate, thus making the drivetrain lighter and preventing misalignment of the shafts and other components.

The main disadvantage of this design is that a defective part generally results in the dismount of the entire nacelle, making its maintenance extremely expensive. Additionally, the gearbox becomes a special and critical component of the system with the capability of holding the rotor, therefore the housing construction must be robust. In this design it is difficult to entirely isolate the reaction forces from the rotor, therefore some of these forces could be transferred onto the other components of the drivetrain, thus reducing their operating lives.[3]

With respect to the design process, the integrated drivetrain configuration generally is limited to one gearbox design. The design process therefore must follow a vertical structure with the wind turbine manufacturers being closely involved throughout the entire design process. To a certain degree this prevents cooperation with different gearbox providers and generally results in the turbine manufacturers owning the gearbox designs; this can result in increased cost. Figure 5 shows the typical configuration for the integrated drivetrain. 


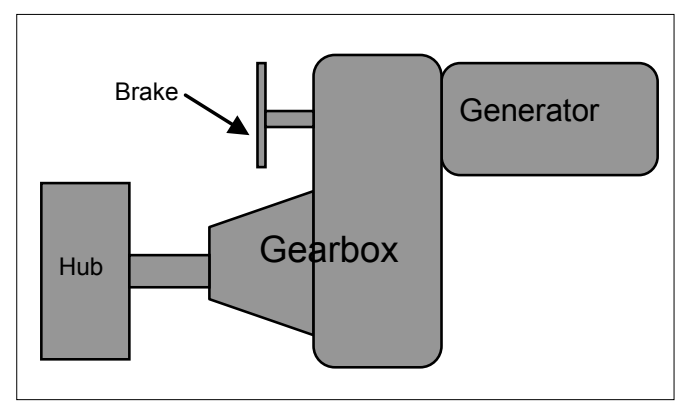

Figure 5. Integrated drivetrain from Wind World W-2700 [3]

\section{Partially Integrated Drivetrain}

The partially integrated drivetrain design is a combination of the modular and integrated design. It follows the modular design in that it uses a bedplate for mounting its components; however some of its components are integrated. The most common configurations are the gearboxgenerator integration or the gearbox-hub integration. In the case of the gearbox hub integration the vertical design process discussed above must be followed, because the gearbox becomes a structural component of the wind turbine. With respect to generator-gearbox integration the design need not be entirely vertical, although a great level of cooperation and integration between the gearbox supplier and generator provider are necessary. These limitations generally result in a slower and more expensive design process. Figure 6 shows the typical configuration for the partially integrated drivetrain configuration.

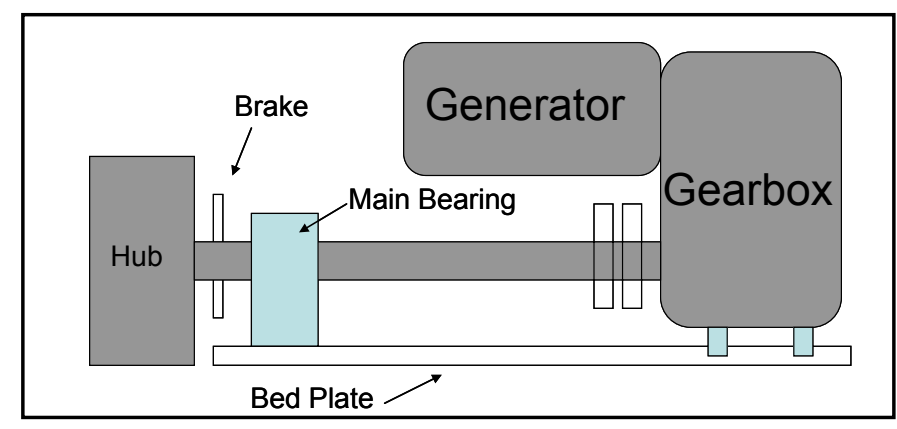

Figure 6. Partially integrated drivetrain [3]

\section{Direct Power Train}

The direct power train concept's main difference is that it uses a different type of generator which eliminates the need for a gearbox. The generator is attached directly to the rotor; it therefore must be capable of producing power at much lower angular velocities. This is achieved by increasing the number of poles, which results in a very large-diameter generator. These large generators typically are cooled with air or a fluid to prevent overheating [4]. The permanentmagnet generator seems to be the most cost-effective approach for this type of generator; its cost, however, is much greater than the generators implemented in the modular configuration.

In the direct drivetrain approach the turbine manufacturer designs the entire drivetrain, resulting in a vertical design approach. The only interaction generally is between the turbine manufacturer and the generator provider, and involves discussion regarding size constraints and coupling requirements. Although this configuration eliminates the prominent gearbox problems, it 
nevertheless seems to be an expensive solution. Consequently, the industry for the most part has maintained the construction of the modular configuration. This concept, however, has been implemented by some European companies such as Enercon (see Figure 7 for a direct drive example).

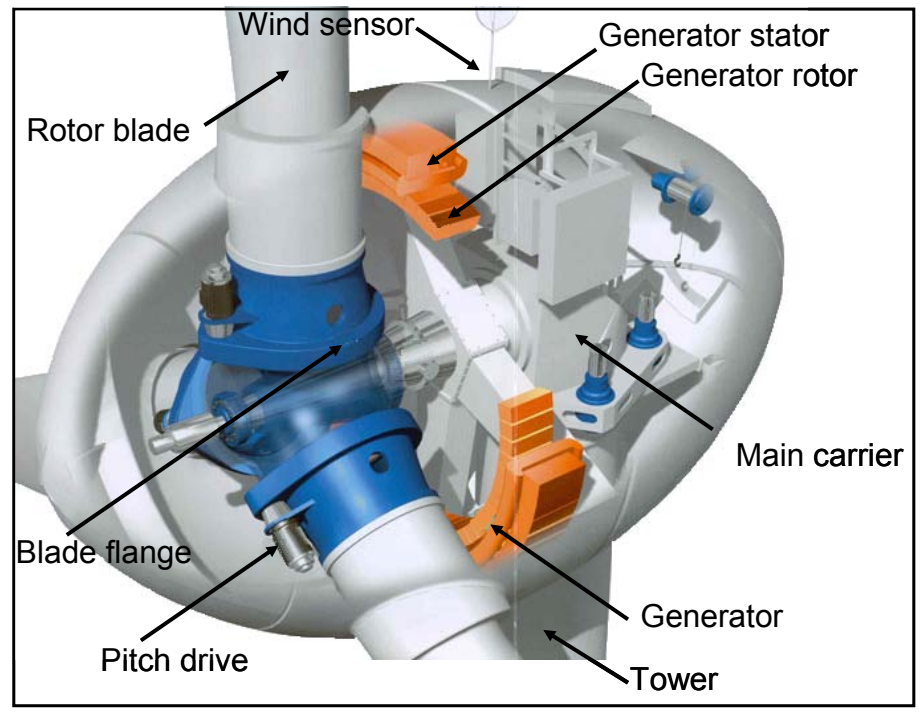

Figure 7. Enercon direct power train [27]

\section{Drivetrain Configuration Comparison}

This section includes a qualitative table which depicts characteristics of the different drivetrain configurations discussed above. Table 1 shows that the modular drivetrain described here is less compatible with the vertical design process. The design process therefore typically involves a great number of vendors for the different components of the drivetrain and gearbox. It is also apparent that the number of parts present in the modular drivetrain increases the difficulty of aligning the different components, but it nevertheless results in the reduction of their individual costs. The integrated and partially integrated designs have fewer parts but their components are specific to a particular design, thus making the non-vertical design process more difficult. The direct drivetrain configuration most favors the vertical design process, because it includes fewer parts and all of the components of the drivetrain are integral to the structure. 
Table 1. Drivetrain Parameter Comparison

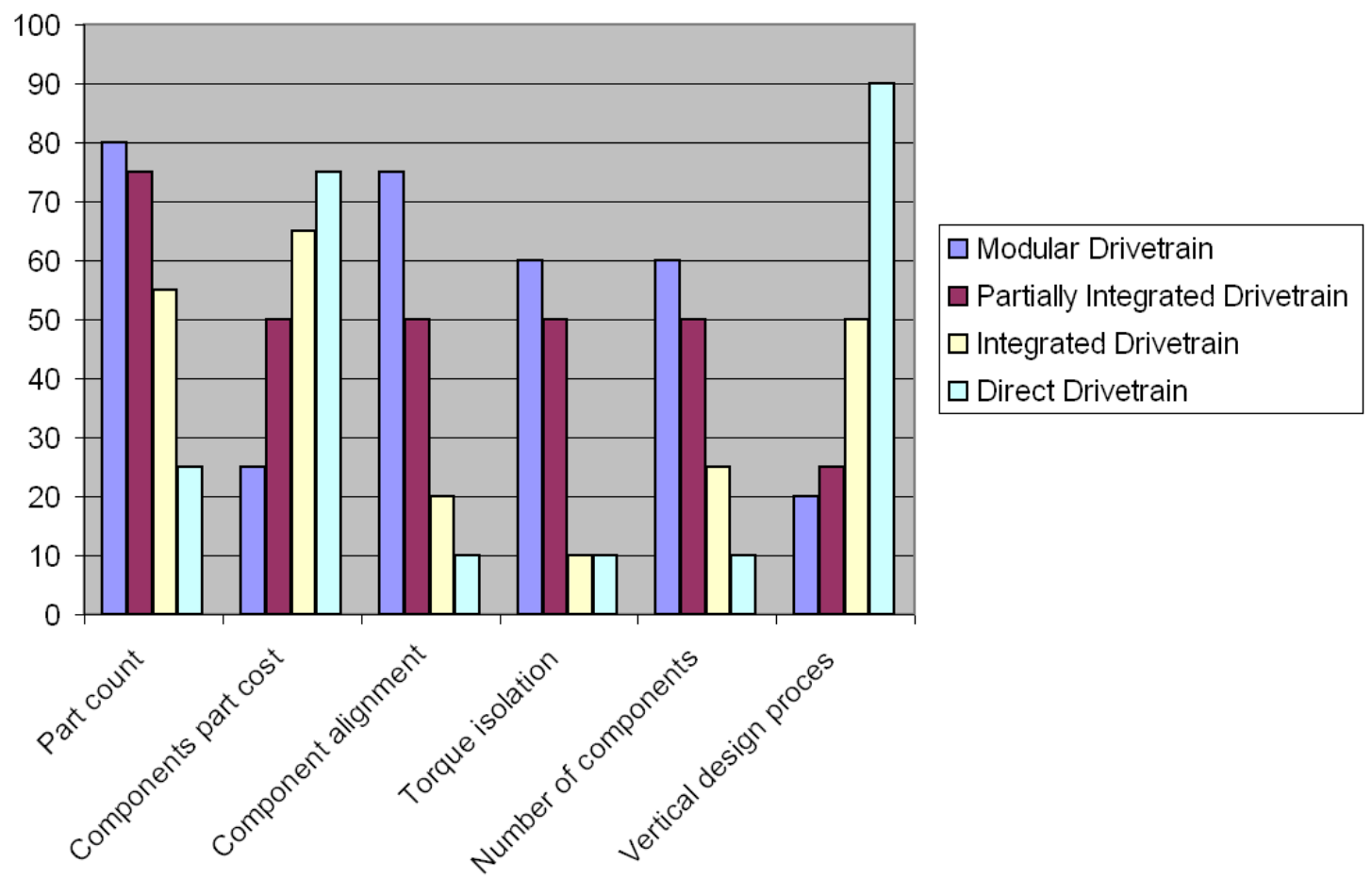

\section{Modular Drivetrain Components}

The following sections describe the different components of the modular drivetrain and briefly explain the challenges that characterize the modeling of each individual component.

\section{The Low-Speed Shaft}

The low-speed shaft transmits loads from the rotor to the gearbox. Its configuration also is intended to minimize the transferred nontorsional load to the gearbox.

The low-speed shaft supports the weight of the rotor and transmits all the reaction forces to the main frame through the main bearing. These reaction forces are composed of all nontorsional loads such as axial thrust from the wind, as well as disturbances from turbulence caused by uneven wind distribution or wind shear.

While operating, dynamical effects such as vibration imbalances from the blades and gyroscopic loads from yaw movement also contribute to nontorsional loading. Although the low-speed shaft is a simple mechanical device it is very important, because the unintended transmission of reaction forces to the other components of the drivetrain could reduce operating life [5].

The simplest model of the low-speed shaft is a totally rigid body that allows no deflections. A better approximation of its real behavior would include its torsional deflection, although it still would be modeled as being infinitely rigid to bending. This approach provides a worse-case scenario for the load transmission to the rest of the component of the drivetrain, because nontorsional loads would be transferred directly with no absorption from the low-speed shaft. A more comprehensive approach includes flexible representation of the low-speed shaft; this truly 
would reveal the contribution of the low-speed shaft to the reduction of the transmission of nontorsional loads to the rest of the drivetrain.

\section{Couplings}

Couplings are mechanical devices used to connect shafts together, and they are capable of transmitting a torsional load from one shaft to the other. The two main types of couplings are rigid coupling and compliant or flexible coupling.

A rigid coupling locks both shafts together, thereby allowing no relative motion between the two shafts. The disadvantage of this type of coupling is that the shaft axis must be precisely aligned to prevent the transmission of forces and moments other than torsion.

The compliant or flexible coupling relies on the use of elastomers or other materials to dampen transmitted torsional loads without shock-load excursions. Another advantage is that to a certain degree they can handle axial, angular, parallel, and torsional misalignment. Thus the assembling tolerances of the system are not required to be as precise. Its dampening capabilities also help decrease torque spikes and vibrations that can damage other components of the drivetrain. The main disadvantage of this coupling is that it can increase the level of backlash in the drivetrain or torsional clearance [6].

\section{Gearbox}

The gearbox is a mechanical device capable of transferring torque loads from a primary mover to a rotary output, typically with a different relation of angular velocity and torque. In the case of wind turbines the gearbox connects the low-speed shaft and the generator; therefore its gear ratio generally is dictated by the requirement of the generator and the angular velocity of the rotor.

In the case of electrical power production with an asynchronous generator, the output of the gearbox (which is connected to the generator) usually operates in the ranges from $50 \mathrm{~Hz}$ to $60 \mathrm{~Hz}$ or $1,500 \mathrm{rpm}$ to $1,800 \mathrm{rpm}$. This depends on the frequency of the grid to which the generator is connected, and on the number of poles of the generator [5]. In many of today's modern machines the generator is able to operate at a greater range of speeds. Regardless of this advantage a speed increment still is required, although it might not be as significant as that needed for the asynchronous generator.

The angular velocity of the rotor can be linked to the tip speed ratio. The tip speed ratio is the ratio of the velocities of the tip of the blade to the wind velocity. An important factor that dictates the tip speed ratio is the area covered by the blades with respect to the area swept by the rotor. As the area of the blades is reduced, the tip speed ratio must be increased. The tip speed is directly proportional to the radius of the rotor, therefore this dimension also dictates rotor angular velocity [3].

Over the years, the power output capacity of turbines has been improved by increasing both the slenderness of the blades and the rotor sweep area. The angular velocities therefore are reduced, creating the need for gearboxes that are capable of handling greater torques and greater gear ratios. Greater torque has led to the development of larger gearboxes - which also have been characterized by the same failures as those experienced by smaller configurations used in smaller machines. Moreover, due to the larger size of the components larger gearboxes also might 
experience material properties scaling discrepancies. Greater gear ratios have caused the use of larger gear ratios per stage and an increase in the number of stages - which has created more complex configurations that are prone to failures.

There are two main types of gearboxes, parallel shaft gearboxes and planetary gearboxes. These are described in the following sections.

\section{Parallel Shaft Gearbox}

Parallel shaft gearboxes are a collection of simple gear stages. Each gear stage is composed of two shafts, a gear, and a pinion. For a gearbox that is designed to increase the angular velocity, the gear is on the input shaft and the pinion is on the output shaft. There is a practical limitation to the maximum gear ratio per each stage, generally due to size constraints and also from possible interface between a very small pinion and a very large gear; this is the reason for using multiple stages in parallel gearboxes [6]. In the case of gearboxes that have large gear ratios the number of parallel stages is minimized, otherwise they would require larger gears which are difficult to produce accurately and are expensive to manufacture in comparison to smaller gears.

In parallel gear stages, the gear interaction can be modeled in several ways (explained below). The parallel stages also have an advantage in that they can be modeled with a minimum number of degrees of freedom. This is because they are attached directly to the gearbox housing and in many cases are attached to the inertial frame. This results in the calculation of the angular velocities of the components of the stage with respect to a fixed frame, thus easing its comparison and understanding.

\section{Planetary Gearbox}

The epicyclic or planetary gearbox offers several advantages compared to the parallel-shaft configuration, including a higher gear ratio in a smaller "package." This compact configuration has the advantage of reducing the overall mass of the gearbox, which is an important requirement for wind turbine gearboxes, because the head mass of the turbine is kept to a minimum.

Additionally, the planetary configuration has the capability of handling greater torque loads. This is because the load is distributed or shared by the number of planet gears, therefore more teeth always are in contact. Another advantage is its geometrical configuration. The input and output have a concentric axis and the same rotational direction; therefore, it is very simple to build multistage planetary boxes that maintain a streamlined and compact design.

The planetary box is more complicated than the parallel shaft, because it is composed of three moving components per stage. These components include the planet gear, the planet carrier, and the sun pinion. The ring gear is also part of the planetary box however it is fixed to the gearbox housing.

The left side of Figure 8 shows the anterior view of a planetary stage. In the outer section the ring gear is represented by circular mesh. Generally the ring gear is fixed to the housing and it is not a moving component. It is an internal gear; the teeth are on the inside of the ring and mesh with the teeth of the planets at all times. The planet carrier is rendered in blue and holds four planets (shown in darker gray). The planet carrier ensures that the proper ring-planet and planetsun center distances are maintained. Its configuration generally is more robust than that shown in the figure, because it must undertake the high-input torque loads. 
The planets are supported on the planet carrier by shafts with bearings, therefore the planets can rotate freely with respect to the planet carrier. The input is provided to the planet carrier which distributes the torsional load among the planets so that the ring-sun interaction can be completed. The posterior side of the planetary stage is shown on the right side of Figure 8. The sun is located in the center of the planets. The load from the planets is transferred to the sun at four points to become the output of the stage.

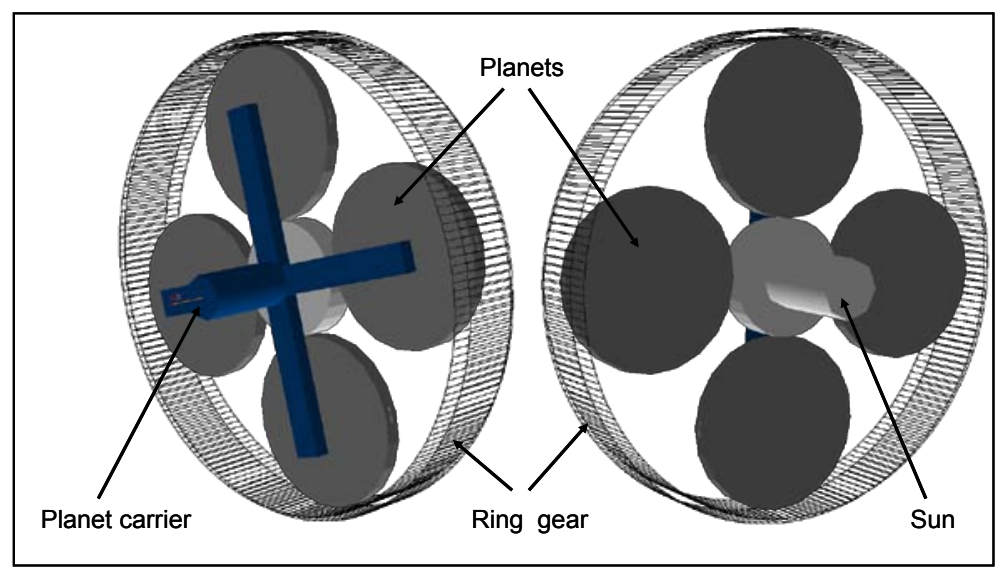

Figure 8. Planetary stage components

Models that depict the planetary gearboxes typically increase in complexity with respect to the parallel shaft configuration; this is due to the interaction of the different moving components and reference frames. A simple example is the behavior of the planet's interaction with the planet carrier; in a simple model the planets are assumed to have only one degree of freedom (rotation). This relation shows the planet's behavior with respect to the planet carrier. At the same time, the planets are interacting with two other components the ring gear (which is fixed to the inertial frame) and the sun (which is constrained to the reference frame but allowed to rotate). This setting leads to difficult data interpretation because the contribution of all the acting components must be considered to be able to understand the behavior. Simple torsional models present their own difficulties, because the planetary stage must be simplified and specific relations among components must be created to simulate the desired response.

As noted, the rotational direction of the input and output of a single planetary stage are the same. This can be seen clearly in Figure 9. For the configuration below, the gear ratio is given by the following expression.

$$
\frac{n_{H s s}}{n_{L s s}}=1+\frac{D_{\text {Ring }}}{D_{\text {Sun }}}
$$

Where $n_{H s s}$ is the rotational speed of the high-speed shaft of output, $n_{L S S}$ is the rotational speed of the low-speed shaft or input, $D_{\text {ring }}$ is the pitch diameter of the ring or its number of teeth, and $D_{\text {sun }}$ is the pitch diameter of the sun or its number of teeth [5]. 


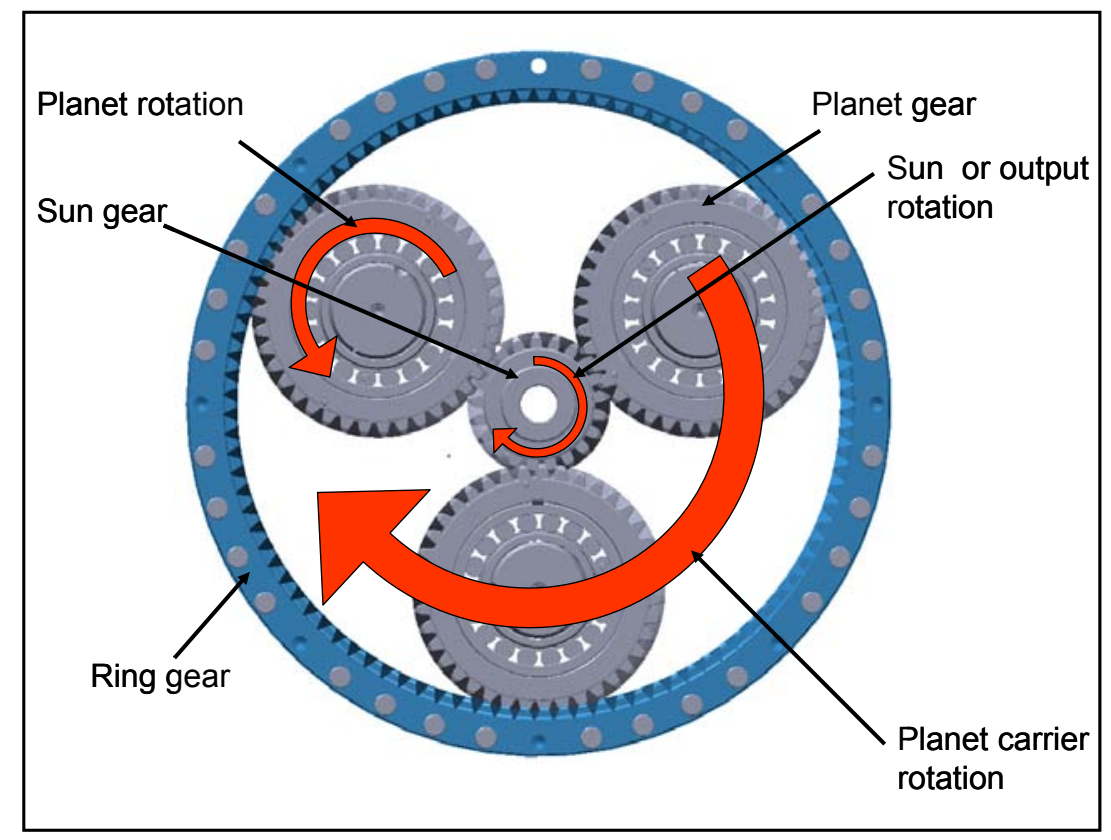

Figure 9. Planetary stage components rotational direction

Notice that, in the expression used to calculate the gear ratio, the only parameter taken into account is the dimensions of the ring gear and the sun. This is what allows for the greater gear ratios, because great dimension differences can characterize these two components.

\section{Brakes}

Brakes are mechanical devices designed to slow or stop a machine. Brakes also are intended to prevent a device from moving after it has been stopped. In wind turbines there typically are two distinctive brake classifications - aerodynamic brakes and mechanical brakes.

\section{Aerodynamic Brakes}

Aerodynamic brakes generally are used in the event of overspeed. Braking during overspeed is achieved by increasing drag at the tip of the blade, which brings the rotor to a safe rotational speed. There are many types of aerodynamic brakes, including variable-pitch blades, deployable blade tips, spoilers, flaps, and brake parachutes. The rotor nevertheless has an effective aerodynamic area. Aerodynamic brakes therefore generally do not fully stop the rotor; however, they still drastically reduce the load of the mechanical brake. The standard braking maneuver typically is to apply the aerodynamic brake and then the mechanical brake; this reduces the inherent loading on the braking operation. The main problem presented by aerodynamic brakes is non-simultaneous deployment of the brakes on each blade, which causes aerodynamic imbalances that can be catastrophic [3].

Aerodynamic braking is an important characteristic of the normal braking event-it greatly reduces the torque excursions that are seen in other braking operations such as the emergency braking maneuver. Simulation of this operation must be performed using the aeroelastic model and integrated into the drivetrain model. Detailed parameters describing the aerodynamic brake are required to create models that properly simulate its behavior. The models generated for this 
report do not include this parameter, and only represent emergency braking maneuvers that are performed solely by the mechanical brake.

\section{Mechanical Brakes}

The most common mechanical brake used in wind turbines is the disc brake. Although it also can be seen on the low-speed shaft, in most designs it is located on the high-speed shaft because the torque loads are much less. The brake is composed of a steel disc that is rigidly fixed to the braked shaft. A set of calipers are fixed to the frame of the shaft is to be stopped. The calipers apply enough force to the disc to stop the shaft. The calipers on wind turbines generally are failsafe, thus the stopping load is applied by springs and the calipers are opened using hydraulics, and if there is a failure in the hydraulics then the brakes are applied [5].

The brake is a frictional device, therefore it can induce a nonlinear torque increment as it is applied to stop the turbine. There also is the possibility of inducing additional vibrations to the gearbox and drivetrain. The modeling of the brake generally neglects these two phenomena, and is represented by a linear torque increment against the rotational motion. This increment peaks at a defined maximum braking torque. The rate at which the peak is reached is of great relevance because it directly dictates the magnitude of the torsional forces induced on the drivetrain to oppose its inertial behavior.

\section{Generator}

Generators are devices that transform mechanical energy into electrical energy. The electrical power is produced by passing a conductor through a uniform magnetic field at a right angle to the lines of electric flux. The voltage generated is a function of the velocity, the conductor length, and the magnetic flux density. The magnetic field used by the generators is obtained by the use of electromagnets or permanent magnets [3].

The induction generator is the most common generator used in the wind industry. This largely is because it has a simple configuration and a low price. Its main disadvantage is that it does not use permanent magnets, thus it has to be connected to the grid to be capable of producing power [7].

The behavior of the generator is mimicked by using an angular velocity-to-torque relationship. For simulations of normal turbine operation, the relationship can be represented by a simple linear relation. In the case of intricate events - such as starting and braking events - a morecomplex relation must be utilized (see Simulation Using FAST_AD, below). An important parameter that must be considered is the inertia of the generator. Due to the gear ratio of the gearbox, slight changes in the rotor velocity result in great changes in the generator rotational velocity. These rapid changes in velocity result in high torsional excitations of the drivetrain.

\section{Control Systems}

The purpose of control systems is to keep the wind turbine operation within permissible rangesespecially in the event of high winds. The parameters to be controlled are rotational speed, torque, and rotor thrust. Basic aerodynamics concepts such as leading edge, trailing edge, lifts and lift drag curve, and flow separation are helpful in understanding the information provided in the following section. Some of these concepts are explained in the Appendix (Aerodynamic Simulation, below). 


\section{Pitch Control}

Pitch control is based on the ability to vary the angle of the blades, which consequently changes the angle of attack of the blade. This allows the rotor to have the optimum angle of attack for different wind velocities, thereby putting the turbine at its optimum performance for a greater range of winds. In the case of high-wind control, an implementation of pitch control is pitching towards feather; meaning that the leading edge of the blade is oriented towards the wind. As a result the angle of attack is reduced, and the lift or driving force generated by the airfoil also is reduced. The main disadvantage of pitching towards feather is that a large angle of rotation is required. This results in a higher-cost system and a slower response toward fast changes in wind conditions. The major advantage of pitching toward feather is that it has the lowest rotor thrust in high winds.

A different application of the pitch control is pitching towards stall. In this case, the blade is rotated in the direction opposite that of pitching toward feather. This increases the angle of attack away from its optimum, as can be seen in the lift and drag force versus angle of attack graph supplied in the Appendix. With a great angle of attack the drag is increased and the lift force is reduced, thus controlling the angular velocity and the amount of torque produced [3].

\section{Stall Control}

In stall control, the angle of the blades is fixed to the optimal angle of attack for the rated wind speed. The angular velocity of the rotor is kept constant by using the torque load of the generator. When the wind speed increases, the inflow relative to the blade's fixed position changes, and the angle of attack increases. Ultimately, this can cause the flow to separate from the blade, increasing drag and reducing lift. This reduction of lift and increase in drag reduces the power output of the rotor, thereby inherently controlling power and torque. The advantage of this design is that it is a passive power-control method and therefore does not involve any moving parts. This makes it a more affordable and popular design in the industry. This system also can react much more quickly to sudden changes in wind speed. The main disadvantage is that the system has greater levels of thrust because the blades cannot be pitched towards feather. The other components of the turbine therefore must be more robust to sustain the greater loads [3].

\section{Pitch Control Versus Stall Control}

The control system has an important role in the loads being generated and transmitted through the turbine. With respect to pitch control, the system's ability to change the blade's angle of attack can reduce thrust generated by the rotor when operating in high winds. The most evident disadvantage is its inability to react quickly to changes in wind speed. This can result in torsional load spikes that must be absorbed by the drivetrain.

The main advantage of the stall-controlled turbine is its simplicity. Due to the small number of control parameters, the influence of the control system can be ruled out as the cause of the observed failures, and a comparison between a great number of subjects can be established. The ability of the stall-controlled turbine to react almost immediately to changes in wind velocity reduces the torsional load spikes that are observed in a pitch-controlled system. The main disadvantage of the stall-control system is the greater thrust loads from the rotor that are imposed on the drivetrain. To reduce the transmission of thrust loads to the other drivetrain components, great care is taken in both the design and during assembly. This is achieved by using a low-speed 
shaft bearing designed to accept the thrust load, assembling it on the shaft with a thrust preload, and designing the bearing location on the low-speed shaft as far forward (upwind) as is practical.

\section{Gears}

Gears are mechanical components that have the ability to transmit motion from one shaft to another. The transmission of motion might not be uniform, and it can include changes in direction and torque. More specifically, gears can be described as toothed wheels which typically are round (but not necessarily) [8]. Gears have evolved from rolling cylinders, to wooden wheels with pegs, to the common gears used today which have specially shaped teeth that are shaped or cut into metal wheels. Figure 10 shows wooden-pegged wheels that were used as gears in earlier eras.

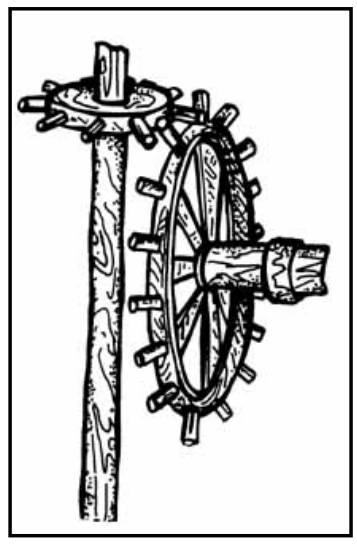

Figure 10. Rudimentary gear set

\section{Fundamental Law of Gearing}

The fundamental law of gearing states that the angular velocity ratio between the gears of a gear set must remain constant through the mesh. The velocity ratio $m v$ can be expressed by equation 2 (below) with the angular velocity input $\omega_{\text {in }}$ and the angular velocity output $\omega_{\text {out }}$ which also can be related to both of the gear-pitch radii.

$$
m v=\frac{\omega_{o u t}}{\omega i_{n}}= \pm \frac{r_{\text {in }}}{r_{\text {out }}}
$$

The positive and negative sign " \pm " accounts for the internal and external gear sets; an external set reverses the direction of rotation between cylinders, and an internal set maintains the same direction. The torque ratio or mechanical advantage $m a$ is the reciprocal of the velocity ratio (equation 3).

$$
m a=\frac{1}{m v}=\frac{\omega_{\text {in }}}{\omega_{\text {out }}}= \pm \frac{r_{\text {out }}}{r_{\text {in }}}
$$

Thus, the gear set essentially exchanges velocity for torque or vice versa. For calculation purposes, the magnitude of the velocity ratio is expressed as the gear ratio [6]. 


$$
m_{G}=|m v| \text { or } m_{G}=|m a| \text { for } m_{G} \geq 1
$$

This exchange of torque and angular velocity is very relevant to the loading condition inherent to the drivetrain. Due to the high gear ratios of the gearboxes implemented in the wind turbines, small changes in the input velocity produce great changes in the velocity of the generator. Similarly, small changes of the applied torque at the output of the gearbox result in greater changes in torque for the initial stages of the gearbox.

\section{Gear Types}

Over the years, many types of gears have been developed based on the industry's needs. These mainly can be classified into parallel axis and non-parallel axis gears. The parallel-axis type can be classified into internal and external spur and helical gears. The non-parallel axis can be classified as bevel, hypoid, spiroid, and helicon, among others. The main gearing used in the wind-energy industry are spur and helical, therefore these are the only ones examined here [8].

\section{Spur Gears}

Spur gears formerly were the type most commonly used in industry due to their simple manufacturing and reduced cost - a consequence of the alignment of the teeth with the axis of rotation. The profile of the tooth is designed to maintain constant ratio from one gear to the other gear. This keeps the tangential velocity from the pinion equal to the gear, ensuring a smooth and quiet operation. There are several types of tooth profiles that satisfy this condition, although for industrial applications the most common is the involute profile.

The involute profile follows the shape of an involute curve, and can be visualized as the curve described by the end point of a taught string uncoiled from a cylinder [6]. This profile is used widely in the industry because of its simplicity, which eases its manufacture. Additionally, it is not as sensitive to the center distances between gears, which reduces manufacturing tolerances of the overall gearing system. The involute profile can operate under greater loads than those of other profiles and, together with the tolerance to center distances noted above, is capable of absorbing the deflections of other components of the system. [9]. Figure 11 shows the shape of the involute curve, originating from the base circle and progressing (always) perpendicular to the tangent of the taught uncoiling string.[6]. 


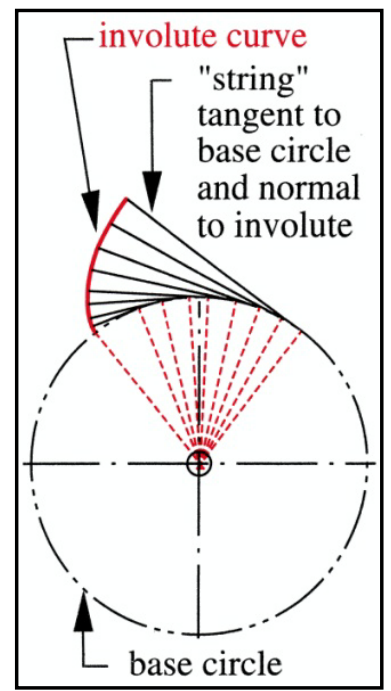

Figure 11. Involute tooth profile [6]

The involute profile is the tooth profile typically used in the wind-turbine industry. The SIMPACK software used to build the progressive models has built-in gear modules capable of simulating tooth-contact interaction. The integration of the tooth behavior into the multibody system models is the main difference between the torsional models and the more comprehensive models.

\section{Helical Gears}

Helical gears have teeth that are cut at an angle relative to the axis of rotation. The advantage of this modification is that these gears have more teeth in instantaneous contact; they also share the load, operate more quietly, and can withstand greater loads. The main disadvantage of this modification is that the gears produce an axial load due to the normal loading of the tooth. This can be compensated for by either using bearings that support axial loading or using a herringbone configuration, in which the gear contains two helixes with opposite angles that cancel the axial forces [8].

Helical gears very commonly are used in wind turbine gearboxes. Planetary stages and parallel stages, for example, are composed of helical gears. The axial forces generated by the helical gears could be responsible for the premature failure of bearings throughout the gearbox, among others things. This is regardless of whether bearings capable of withstanding axial loading are used.

\section{Involute Gear Tooth Nomenclature}

Knowledge of general gear nomenclature is relevant here, particularly because the nomenclature is used to define the mesh stiffness for the Stage 2 model. The nomenclature is used in Figure 12. [6]

- Base circle - the cylinder from which the involute curve originates.

- Pitch point - the point between the axes of the gear set at which the teeth contact. It defines the dimension of the pitch circle. 
- Pitch circle - typically divides the tooth profile in two sections, generally at five-ninths of the height of the tooth (in accordance with the American Gear Manufacturing Association specifications for standard tooth proportions). The portion of the tooth extending from the pitch circle is called the addendum and the section below is called the dedendum.

- Line of action - a line described by the points of contact of the gear tooth as it rotates. The line of action is tangent to both base circles of the gear set and passes through the pitch point.

- Pressure angle - the angle between the tangent of the pitch circle and the line of action.

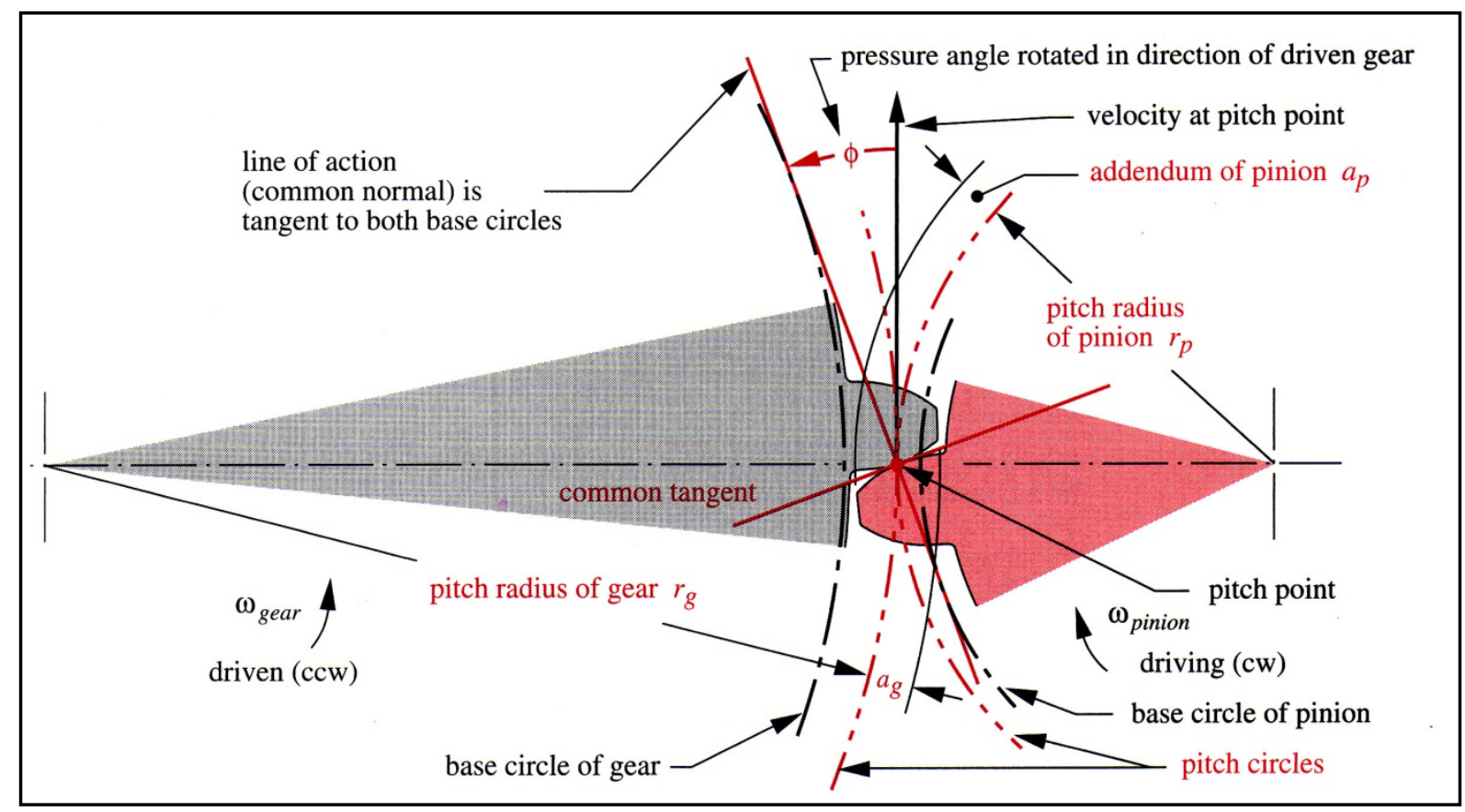

Figure 12. Involute tooth nomenclature [6]

\section{Gear Failure Modes \\ Wear}

Wear is a surface phenomenon whereby metal is removed or worn away more or less uniformly from the contacting surfaces of the gear [10]. This phenomenon is highly dependent on lubrication, more specifically on factors such as oil-film thickness and oil cleanliness. Additionally, the ground surface roughness of the tooth flanks plays an important role. [8]

Polishing is a mild type of wear whereby the surface roughness asperities of the contacting surfaces are reduced and the surfaces become smooth and mirror-like [10]. The process rarely results in failures (although, if allowed to progress, it can result in failure). Polishing generally occurs in low-speed applications in which the elastohydrodynamic lubrication is insufficient. 


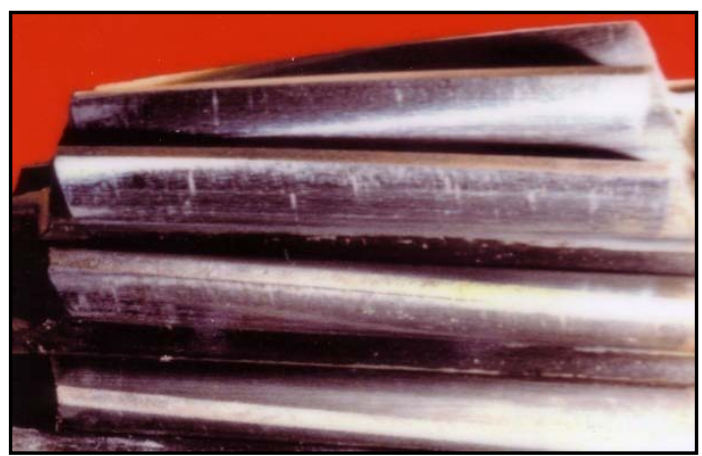

Figure 13. Moderate tooth wear [36]

\section{Moderate and Excessive Wear}

Wear is proportional to the sliding velocity. Sliding velocity increases near the tip and root of the tooth, thus increasing the wear in that area. At the pitch line sliding practically is nonexistent, therefore the wear is much less in that area. Moderate surface-hardened gears are more prone to prominent wear. Excessive wear (see Figure 13, Figure 14) is a progression of moderate wear, at this point the progression towards failure is much faster. This is because a large portion of the tooth has been removed, thus reducing the fatigue life of the tooth and deforming the tooth profile; this results in excessive spalling [8].

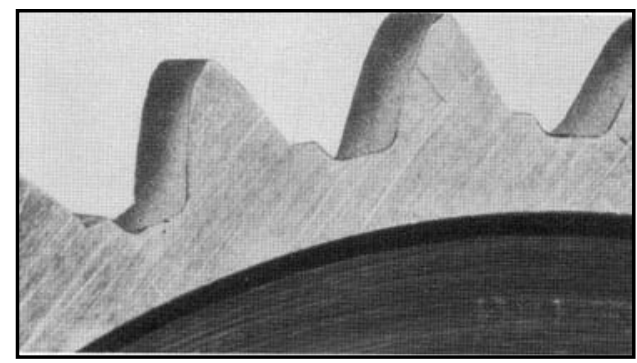

Figure 14. Excessive tooth wear [10]

\section{Abrasion}

Abrasion (see Figure 15) is caused by particles that have hardness near to or greater than the hardness of the gear and which are suspended in the oil film. To create the abrasion the particles also must be larger in diameter than the oil film. Abrasion appears as small grooves that are carved outwardly from the axis of the gear. It can be prevented by using a filtering system that eliminates larger particles from the system [8]. 


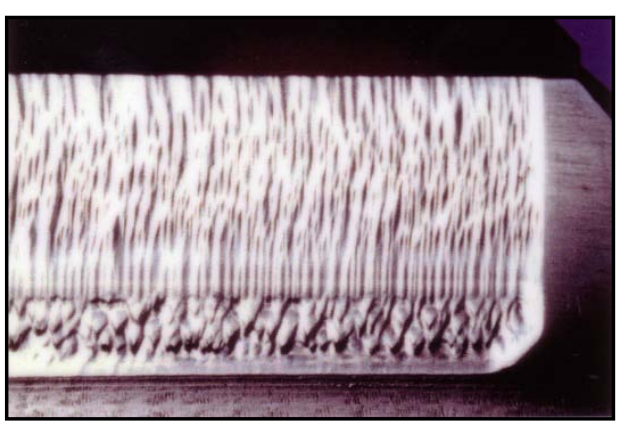

Figure 15. Gear-tooth abrasion [36]

\section{Tip Root Interference}

Interference (see Figure 16) can be caused by poor profile design or by incorrect center distances. Its appearance is much like that of abrasion, although it only affects the area from the tip to the pinion tooth and the root of the gear tooth [10].

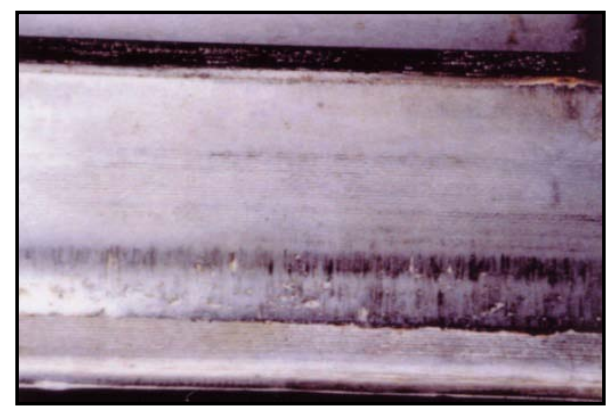

Figure 16. Tooth interference damage [36]

\section{Surface Fatigue}

Surface fatigue is generated by the loading and unloading of the tooth face. The failure originates under the surface of the tooth when the endurance limit of the material is exceeded. Surface fatigue generally is not catastrophic during its early stages, although it typically progresses and ends in catastrophic failure, depending on the loading conditions [10].

\section{Micropitting}

Micropitting (see Figure 17), also known as initial pitting, is characterized by the formation of small pits on the surface of the tooth. The size of the pits ranges from $0.38 \mathrm{~mm}$ to $0.76 \mathrm{~mm}$ ( 0.015 in to $0.030 \mathrm{in}$ ). The failure originates at localized overstressed areas and subsequently is followed by a redistribution of the load. This redistribution can result in the cessation of the pit production. The subsequent operation of the gear generally results in the polishing of the pitted area, improving its appearance. In many cases, if the tooth surface geometry is disrupted by the initial micropitting, then the pitting progresses and evolves into macropitting and tooth breakage. 


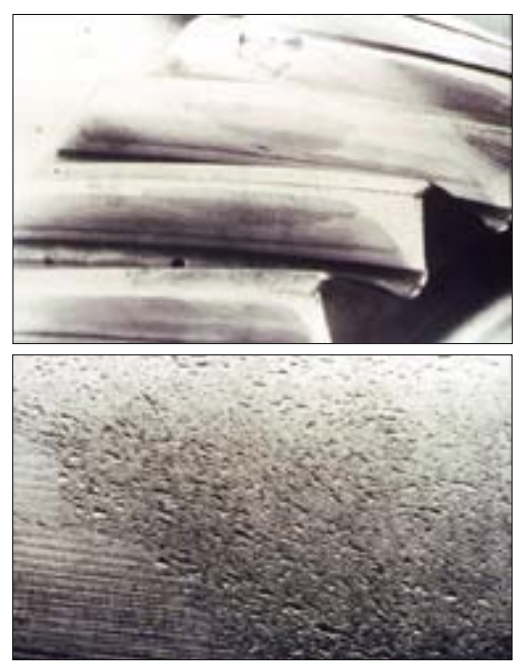

Figure 17. Surface fatigue micropitting [34]

\section{Macropitting}

Macropitting (see Figure 18), also known as destructive pitting, is a progression of micropitting and results in the destruction of the tooth profile. It usually is a consequence of poor load distribution and high Hertzian stress [8]. Its appearance is similar to that of micropitting, but it is larger and has a more irregular shape [10].

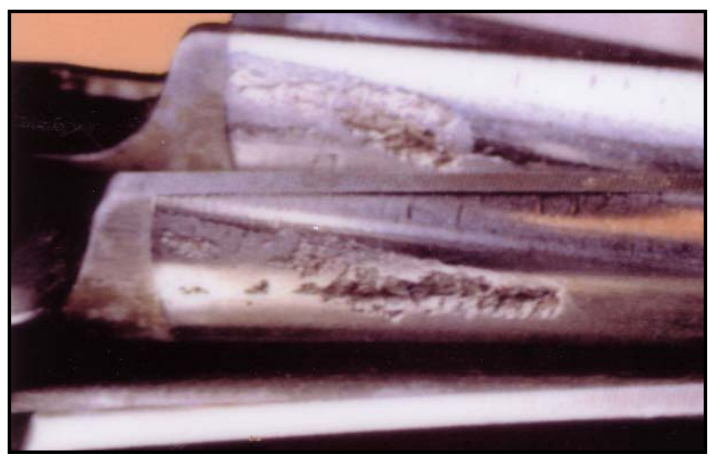

Figure 18. Surface fatigue macropitting [36]

\section{Spalling}

Spalling (see Figure 19) is similar to macropitting except that the damage is quite extensive, more irregular, and quite shallow. It is more prominent in materials of medium hardness, although it also can occur in highly loaded fully hardened materials [10]. 


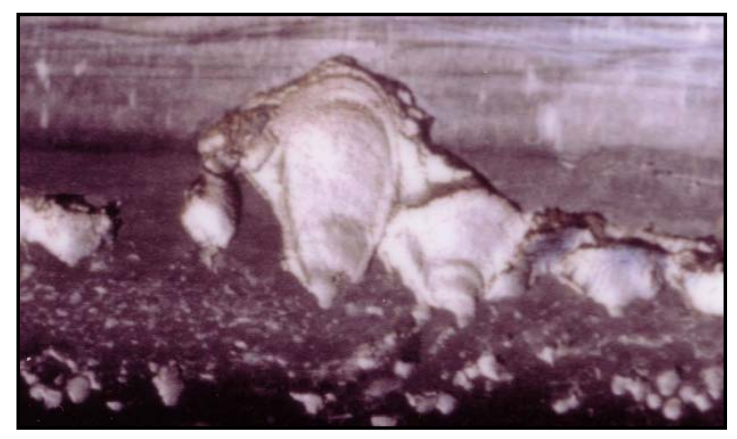

Figure 19. Surface fatigue spalling [36]

\section{Crushing}

Crushing failure mode occurs in surface-hardened or case-hardened gears, when the endurance limit is exceeded below the hardened region. The failure usually is in the form of a crack that, once it originates (typically in the interior where the metal is softer), tends to travel towards and through the surface-hardened portions of the gear. [10]

\section{Plastic Flow}

Plastic flow (see Figure 20) occurs from the yielding of the surface and subsurface of the tooth caused by high-contact stresses. Generally, gears manufactured using softer material are prone to this type of failure; however hardened gears under heavy loads also succumb. [10]

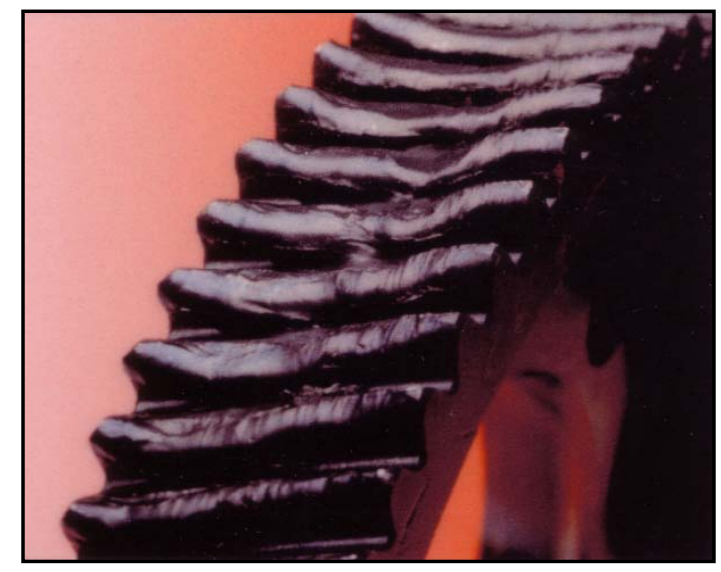

Figure 20. Plastic flow failure [36]

\section{Fracture}

Fracturing (see Figure 21) can be produced by fatigue, tooth bending, or overload. It usually results in catastrophic failure and its progress is more rapid than the mechanisms discussed above [10]. This type of failure results in the immediate loss of serviceability, and usually occurs with little or no warning. 


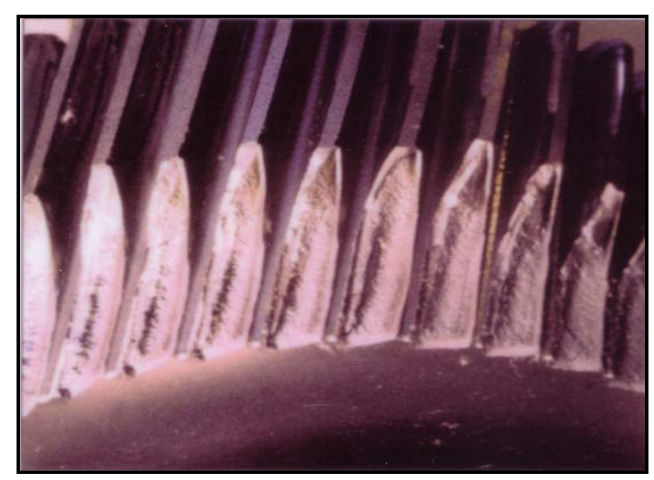

Figure 21. Gear-tooth fracture [36]

Bending fractures generally originate at the root of the tooth; cracks start and propagate, breaking the entire tooth or a portion of it. This type of fracture can show signs of fatigue such as beach marks or fretting corrosion and a point having a jagged appearance, which is the last point to break.

Overload breakage typically has a fibrous or stringy appearance that generally shows evidence of being pulled apart abruptly and rapidly. No signs of fatigue are present [10].

\section{Bearings}

Bearings are devices that allow constrained motion, generally rotational motion. Over time, these devices have evolved to a variety of configurations that are dependent upon their application. The most common bearing used in the wind industry is the rolling element bearing. These bearings are chosen due to their low friction and high load capacity. The wind industry, however, now is moving toward the use of roller bearings. This is because, for larger bearings, roller bearings have a higher capacity and are more cost effective. In the case of axial loading, the use of tapered roller bearings usually is implemented.

\section{Bearings Failure Modes}

The bearing failure mechanisms are quite similar to the gear failure mechanisms discussed in this report. The failures can be classified into two subdivisions-lubrication failures and surface fatigue failures.

Surface fatigue failure (see Figure 22) generally is progressive and can be classified into micropitting, macropitting, and spalling. Typically the raceway fails first with respect to the other components of the bearing under surface fatigue. The bearings give an audible indication of pitting. Pitting increases the vibration of the system, and can progress and result in spalling or fracture of the rolling elements. Spalling produces a large amount of debris [6]. 


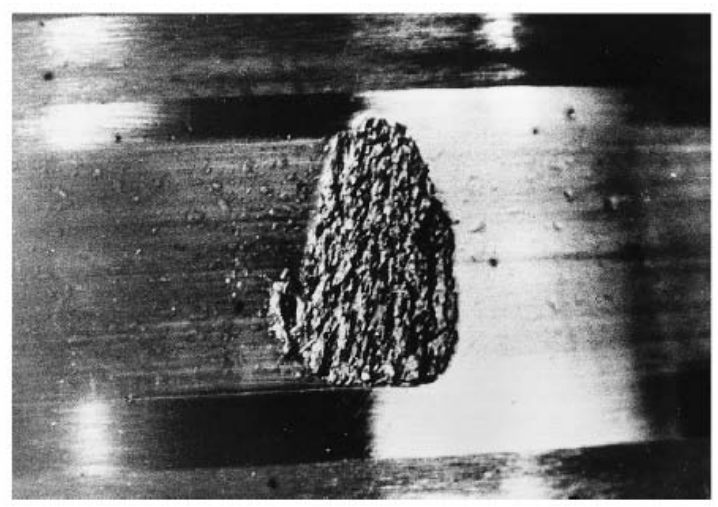

Figure 22. Surface fatigue on bearing raceway [33]

Lubrication failure is the result of the absence of the required lubricant film thickness that is needed to prevent the contact of the rolling elements and the raceways. The lack of the required film thickness results in metal-to-metal contact and causes the bearing to overheat. The symptom typically is discoloration of the roller elements, rings, and cages. In many cases, high temperatures also can degrade or destroy the lubricant [33]. Overheating (see Figure 23) results in the loss of bearing-material hardness, which eventually causes failure. In the case of wind turbines, many of the bearings operate at low rpm, which consequently induces wear due to a loss of film thickness or complete loss of elastohydrodynamic suspension. This mechanism, however, is not as catastrophic and does not progress as rapidly as overheating.

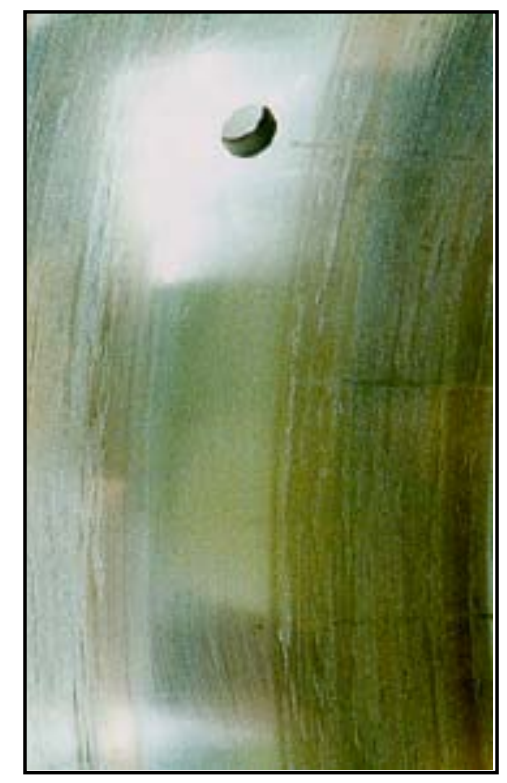

Figure 23. Adhesive wear produced by overheating [33]

\section{Gear and Bearing Failures in Wind Turbines}

The maturation of the gearbox-design process that currently is used in the wind-turbine industry has reduced gearbox failures that stem from gear failures. The main driving failure mechanism in the gearboxes currently used in the industry is surface-fatigue failure of the bearings. The debris 
produced by this failure mechanism inherently causes the abrasion of other components of the gearbox.

There are characteristic loading conditions that drive this surface-fatigue failure mechanism in the bearing, including misalignment and reverse axial loading. Other parameters that influence the failure of bearings can include the bearing fitting either loosely or tightly with the housing, which can result in fretting.

Nevertheless, gear failures also occur independent of bearing failures, although not as commonly. The most common gear failures in the industry include wear from poor lubrication (generally seen in the planets due to their low rpm), abrasion, and surface fatigue initiated by the debris generated from bearing failures. Other common causes of gear failures include toothinterference, poor tooth load distribution produced by misalignment, and changes in center distances due to gearbox housing compliances.

The models developed in this report cannot accurately predict any failure related to lubrication such as wear; however they can predict the loading conditions produced from misalignment, changes in center distances, backlash, and high loads generated from the interaction of dynamical components. The prediction of these parameters reveals important information that can be used for the life-analysis of the different components. Table 2 shows the capabilities of the models with respect both to load-case prediction and to the models' progressive complexity. The model of highest complexity presented in this table is a finite element model. The advantage of this model is that it can generate loading conditions, as well as analyze internally the stress and life-reducing parameters of the gears and bearings. The main disadvantage of this approach is the greater computational time due to the great number of degrees of freedom.

Table 2. Failure Mechanisms and Model Refinement Relation

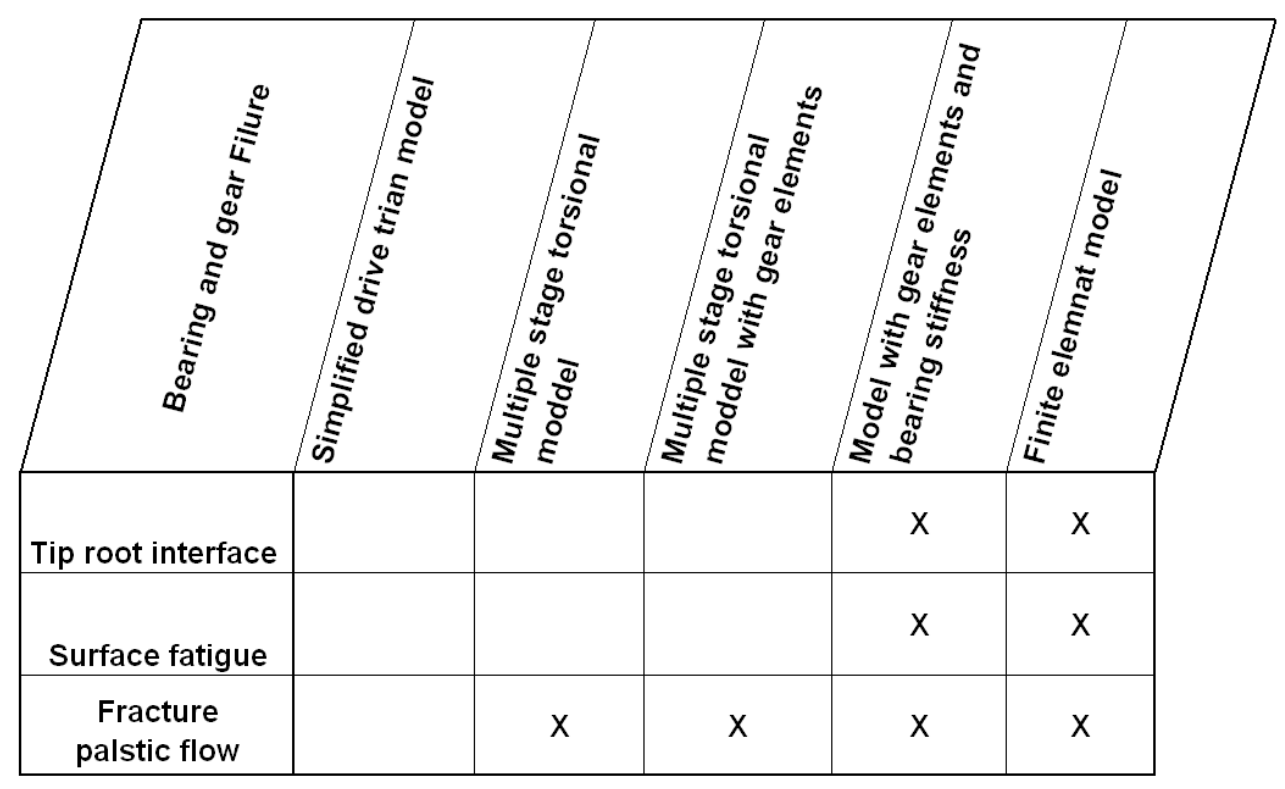




\section{Simulations Using FAST_AD}

FAST_AD is a medium-complexity code used for aerodynamic analysis of horizontal axis turbines, and which allows the simulation of turbines with two or three blades, as well as a teetering or rigid rotor [5]. FAST (Fatigue, Aerodynamic Structures and Turbulence) models a wind turbine by combining rigid and flexible bodies. The flexible bodies include the blade tower and drivetrain shaft. The code uses Kane's method to solve the equations of motion that describe the behavior of all independent components. The model employs generalized coordinates which result in the elimination of constraint equations, thus reducing the computational time [18]. The analysis allows for 14 degrees of freedom including multiple tower-bending modes. [5]

FAST_AD is operated by means of a number of input files that describe the properties of the turbine as well as its aerodynamic characteristics. The main file or primary file describing the turbine has a series of flags that can be changed to true or false to match the simulated turbine. Parameters of note described in the primary file include simulation controls, which control the numerical parameters of the simulation; the turbine control, which describes the controlling parameters of the turbine such as start and stop times for the generator and the brake as well as for the control of pitch angles; turbine configuration, which describes masses and physical dimensions of the turbine stiffness and gear ratios; and finally parameters which describe two generator models. Additionally, this file contains the required names for other input files that the code must use as the simulation progresses.

FAST can incorporate user routines for the controls of the turbine and to describe the generator, in case the predisposed models provided by FAST do not replicate the behavior of the turbine closely enough. The code also handles routines for simulating turbulent wind input to the rotor and handles routines to determine aerodynamic loads.

\section{Multibody System Simulations}

Rigid body motion is the motion of a body that changes position in space over a period of time. The motion is said to be rigid because it is not subjected to any strain, thus the distance between two points on the body remain constant. The change in position includes both translation and rotation. This type of motion is a statically indeterminate motion, therefore a supplementation of Newton's law with Euler's law is used to describe it.

The motion of the body is described by equations which refer to an inertial frame. An inertial frame is a coordinate system that translates with uniform velocity and constant orientation with respect to the stars [13]. The equations describe the motion of a body with respect to time, and are constructed in relation to the number of degrees of freedom of each body and the overall system.

Two types of coordinates can be used to create the equations of motion - absolute and relative. Absolute coordinates relate each individual body to the inertial frame, which results in consistently having the maximum number of dimensions in the equations of motion. Consequently, the time integration is heavier and more time consuming. Relative coordinates rely on a kinematic tree structure in which the degrees of freedom of each body are specified with respect to the previous body, but only the first body is related to the inertial frame. This 
approach results in equations of motion with minimal coordinates, thus making the time integration more efficient.

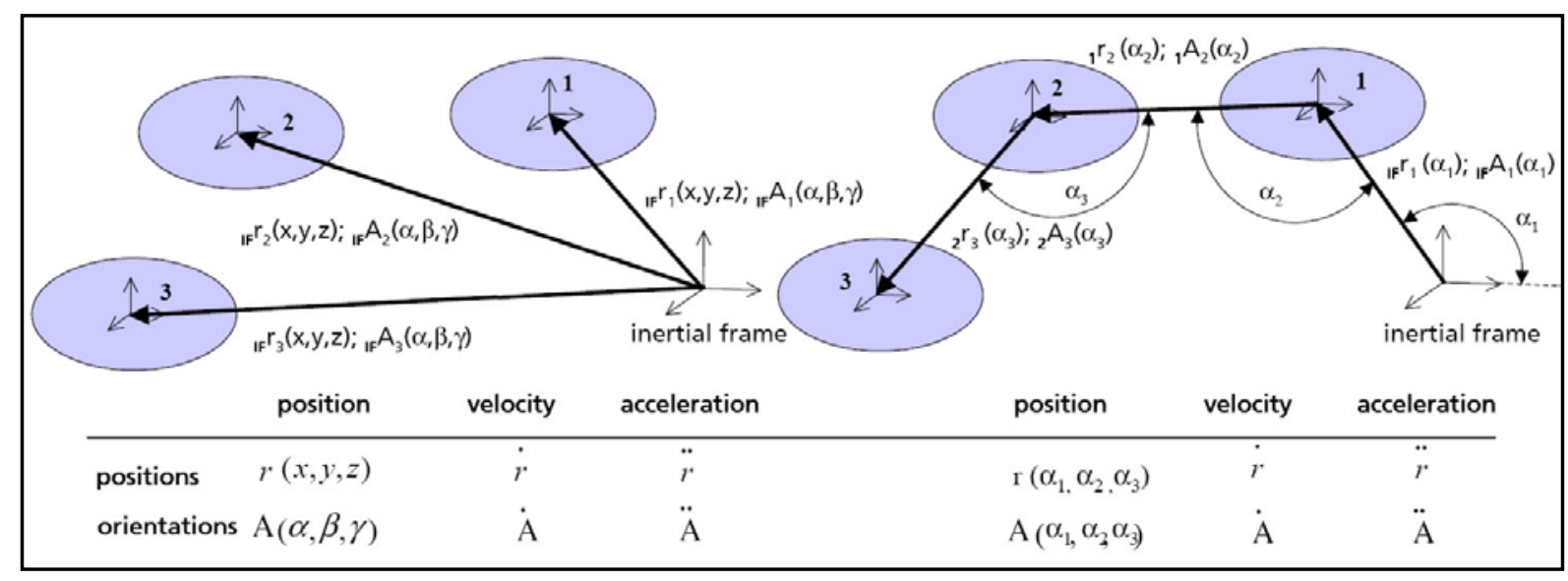

Figure 24. Absolute and relative coordinates [14]

Figure 24 shows both types of coordinate systems - the absolute coordinate system is on the left side, and the relative coordinate system is on the right side. [14] In short, the multibody-system principle decomposes the system into free-body diagrams, enabling the degrees of freedom can be depicted. The kinematic loops are defined describing motions in relation to adjacent bodies by way of joints, constraint, and forces. The equations of motion are formulated and integrated over time.

\section{Simulation with SIMPACK}

SIMPACK is a multibody system simulation commercial code, originally created by the German Aerospace Center DLR as a successor of MEDYNA. In 1993, INTEC GmbH was created to develop SIMPACK. Over the years it has matured to meet the industry multibody simulation demands. [29]

This software is operated via a graphical user interface (GUI). The user inputs parameters describing the system to be simulated, such as mass and inertias of each body, and then can create kinematic loops by applying the pertinent joints, constraints, and forces. SIMPACK creates the equations of motion internally and allows the user to choose different options for the time integration. To obtain visual animations of the interacting system, the user also can input threedimensional primitives which describe the geometries of the interacting bodies.

SIMPACK has a force element library containing specific force elements tailored for different areas of the industry, including specific elements designed for the drivetrain simulation (explained in more detail in the following section).

The SIMPACK software has parameterization capabilities enabling storage of the input parameters of an arbitrary system on a database that contains a number of input files. These input files can be modified and stored separately, depicting a particular condition for a particular case. This feature simplifies the iterative process of data comparison and allows the system to be redimensioned to other similar systems. [14] 


\section{Force Element Description \\ SIMPACK Force Element FE:12, Torsion-Spring Suspension (Force Law Based on the Joint State Quantities)}

The torsional spring force element provided by SIMPACK (see Figure 25) utilizes the state quantities to simulate the spring response. Its main limitation is that it only can operate with a rotation joint with a single degree of freedom. It has the capability of simulation of linear and nonlinear responses, as well as the possibility of describing a clearance or a prescribed nominal torque.

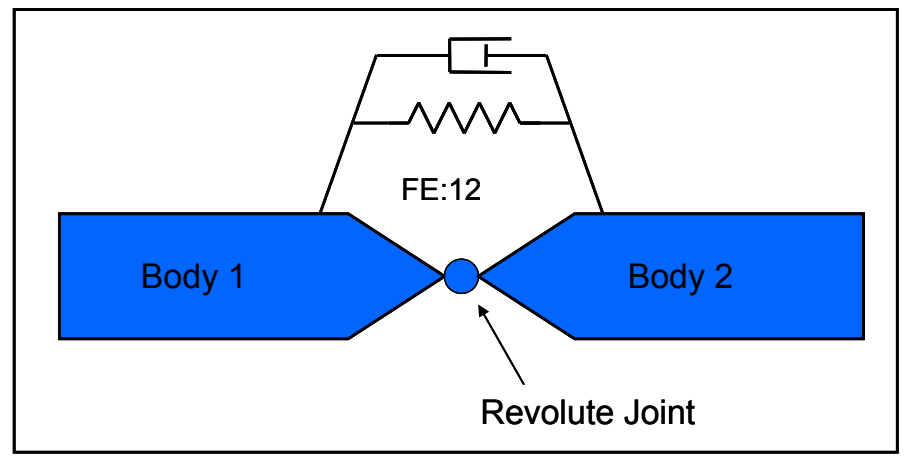

Figure 25. FE_12 spring damper

Input parameters of the force element include the joint identification, from which joint states are used for the calculations. The spring's torsional stiffness and damping, the pre-stress moment, clearance, and angle of zero torque also can be defined. In the case of nonlinearity, a function describing the nonlinear behavior can be used for the stiffness and damping parameters. The output of the force element can be seen as the joint forces on which the element is acting.

\section{SIMPACK Force Element FE:14, Gearbox with Elastic Transmission}

This force element allows the representation of a gearbox as an elastic transmission. It is capable of calculating the input and output shaft torque as well as the velocities. The main limitation is that both input and output shafts must be connected to a common body or housing. The force element allows the user to choose the orientation of the input shaft and output shaft arbitrarily, as well as to change the direction of rotation to account for gear interaction. The joint used for the shaft housing interaction must have only a single degree of freedom, as this force element also uses the joint states for the calculation of the output values.

The input parameters for the force element are: the orientation of the axis where the input and output moment is applied, the label of the connecting joints, the gear ratio, and the stiffness and damping of the system, as well as a pre-stress moment. The outputs of the force element are the torques from both shafts and the difference in angular rotation.

\section{SIMPACK Force Element FE:225, Component Force Element}

The FE:225 force element is capable of modeling tooth-gear contact by considering forces and moments generated in the gear mesh. The force element accounts for dynamical changes in the distance between the axes of the pairing gears. It adds the force contributions of each contacting tooth, considers the number of teeth in contact, and calculates the forces and torques (see Figure 26). The stiffness is calculated depending on the nonlinear relation of the contact point on the 
flank, as well as the face-width of the pairing gears. The forces are calculated from the penetration of the contact points into the tooth profiles, which lay at the line of action. The force element also is capable of reproducing the interaction of gears of different materials and backlash for reversal of direction.

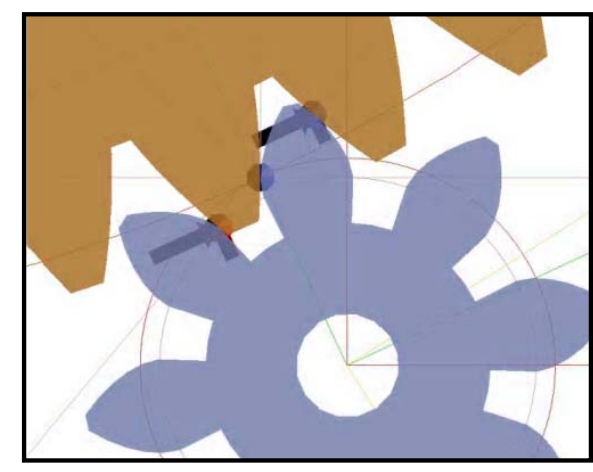

Figure 26. FE:225 point of contact [38]

The force element uses standard gear-wheel physical parameters described from a threedimensional gear-wheel primitive. The primitives are limited to involute tooth geometry; although they can account for spur or helical gears as well as internal and external gears. Some parameters describing the primitive are normal module, profile shift factor, addendum, dedendum, backlash factor, number of teeth, face width, and bore diameter. The force element then requires material properties of the gear wheel, including stiffness model, friction model, tip relief factor, and damping coefficient. The main limitation of the force element is that the axes of the pairing gears must be parallel.

The output of the force element includes: rotational velocity, difference in angle, difference in velocity, contact region, contact stiffness, total circumferential force, axial force stiffness, damping force, friction force, pitch points, angle of attack, dynamic transmission error, penetration velocity, and damping.

\section{Simulation Theoretical Input Parameters}

\section{Shaft Torsional Stiffness \\ Mechanical Interaction}

There is no slip in the interaction of the gear or the pinion with the shaft. The diameter of the gear is much larger than the shaft, therefore the section of the shaft under the gear or the pinion is considered to be infinitely stiff. Consequently, the length of the shaft used to calculate the shaft stiffness is the internal distance between the gears.

The shaft is subjected to pure torsional loading, and no axial bending or direct shear load are present. The cross section of the shaft remains a plane and perpendicular to its axis. The material of the shaft is homogeneous, is isotropic, and obeys Hooke's law. The stresses realign on the elastic limit and its behavior is purely linear. The interaction of the boundary conditions of the bar doesn't influence the behavior of the bar. Initially the shaft is straight. [6] 


\section{Hooke's Law}

The mechanical behavior of any material can be described by a relation between stress and strain. The described curve varies its shape depending on the material properties such as ductility and hardness. For all materials, the initial stages of this curve have a quasilinear behavior. This quasilinear behavior can be said to be linear for all materials; this generalization is known as Hooke's law. The magnitude of this quasilinear stage is dependent also on the material properties. Hooke's law can be represented by the following equation.

$$
\sigma=E \varepsilon
$$

Where $\sigma$ is stress, $E$ is the proportionality constant called the elastic modulus or Young's modulus. $\varepsilon$ is strain and dimensionless, therefore $E$ has the units of stress. Graphically $E$ can be said to be the slope described by a straight line from the origin to a point $\mathrm{A}$ on the stress versus strain curve. Point A lies at the end of the quasilinear portion of the curve (see Figure 27).

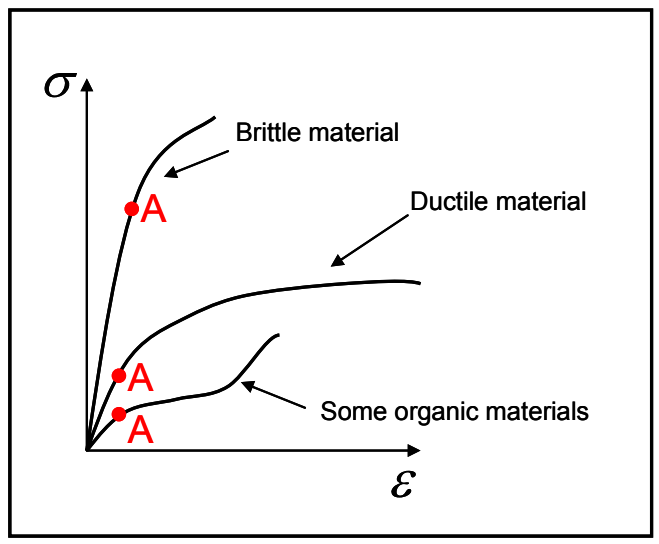

Figure 27. Stress versus strain representative curves [12]

The linearization noted above is of great relevance here, because all the parameters used to create the progressive models are described with a linear response.

\section{Shear Strain Relationship}

A relation between shear stress and strain can be formulated in a similar manner. In this case the angle $\gamma$ is used to describe the deformation or strain of the element under pure shear load. In Figure $28, \gamma$ describes the angle to which the element has been deformed from its original configuration. 


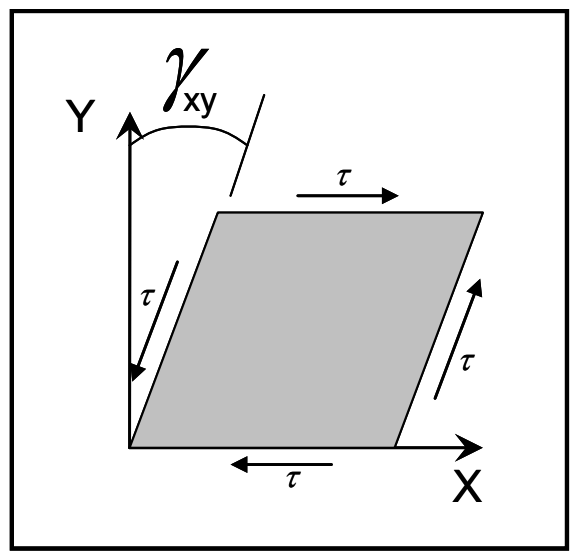

Figure 28. Shear deformation [12]

Hooke's law can be extended to this type of loading, creating a liner relationship between shear stress and angular deflection. This can be represented by the following equation.

$$
\tau=G \gamma
$$

Where $\tau$ is the shear stress, $\gamma$ is the angular deformation, and $G$ is the proportionality constant (shear modulus of elasticity). [12]

\section{Torsional Deflection of a Circular Shaft}

If a circular shaft is deformed under torsion, then the only deformation is the angular displacement from each cross section to the subsequent cross section. The sum of these displacements gives the angle $\varphi$ or the total angular deflection of the rod. Examining an infinitesimal rectangular element $(d A)$ bounded between two cross-sections reveals that the only deformation that the element experiences is the angle of its corners. These differ from the original $90^{\circ}$; essentially the element is undergoing pure shear and, consequently, the angle is $\gamma$. As shown in Figure 28, the relation in $r_{1} \varphi=l \gamma$ can be established. Substituting Hooke's law, $\tau=G \gamma$, results in the following.

$$
\tau=\frac{\varphi G r_{1}}{l}
$$

Where $\tau$ is the shear stress, $\varphi$ is the torsional deformation of the bar, $G$ is the proportionality constant (shear modulus of elasticity), $r_{1}$ is the radius of the cylindrical side of the element, and $l$ is the length of the bar. Because $\varphi, G$, and $l$, are constants, the value of the shear stress $(\tau)$ varies directly with the radius. The applied torque can be calculated by integrating over the crosssectional area, yielding the following.

$$
T=\int_{0}^{r} \tau r_{1} d A
$$

The shear stress changes linearly from the axis of the bar, so the following is a constant.

$$
\tau / r_{1}
$$


Applying this property to the previous equation yields the following.

$$
T=\int_{0}^{r} \frac{\tau}{r_{1}} r_{1}^{2} d A=\frac{\tau}{r_{1}} \int_{0}^{r} \frac{\tau}{r_{1}} r_{1}^{2} d A=\frac{\tau}{r_{1}} I_{p}
$$

The integral

$$
\int_{0}^{r} r_{1}^{2} d A
$$

is substituted by the variable $I_{p}$, which is called "polar moment of inertia" and is the ability of an object to resist twist; in this case it is for a circular solid rod.

$$
I_{p}=\frac{\pi d^{4}}{32}=\frac{\pi r^{4}}{2}
$$

Substitution of equation 7 into equation 8 yields the following.

$$
\varphi=\frac{T l}{I_{p} G}
$$

The units of the angular displacement are in radians. [9] The general equation for the calculation of the stiffness is as follows.

$$
k=\frac{T}{\varphi}
$$

If the previously obtained deflection is applied, it shows that the stiffness is solely dependent on the geometry and material description. It can be represented by the following equation.

$$
k=\frac{T I_{p} G}{T l}=\frac{I_{p} G}{l}
$$

\section{Torsional Free Vibration}

Vibrations refer to oscillatory mechanical motion from an equilibrium point. These oscillations can be periodic or random. The natural frequency or the frequency of free vibration is the frequency at which the system vibrates when there is no external excitation. A particular system can have more than one natural frequency; however, for the purposes of this report, only the lowest, undamped natural frequency is considered. In the case of lateral vibration, the natural frequency is characterized by the stiffness mass of the system. In the case of a torsional vibration, the same relation is used except that the relation involves torsional stiffness and the mass moment of inertia. It can be expressed by the following [6].

$$
\omega_{n}=\sqrt{\frac{k_{t}}{I_{m}}} \mathrm{rad} / \mathrm{sec}
$$


Where $\omega_{n}$ is the lowest free vibration frequency in radians per second, $k_{t}$ is the torsional stiffness in $\mathrm{N}-\mathrm{m} / \mathrm{rad}$, and $I_{m}$ mass moment of inertia is in $\mathrm{Kg}-\mathrm{m}^{2}$. If the natural frequency is provided in Hertz, it can be changed to radians per second using the following expression.

$$
\omega_{n}=2 \pi f_{n}
$$

\section{Torsional Free Damped Vibration}

In practice, a real system oscillatory motion is observed to die down gradually. To simulate this behavior, a viscous damper is introduced to the free vibrating system and it acts as a force proportional to the velocity of vibration. The viscous damper is purely a mathematical contrivance to suit the experimental observation. The addition of the damping factor to the equation of motion for a simple spring mass system can be expressed as follows.

$$
m \ddot{x}+c \dot{x}+k x=0
$$

Where $c$ is the damping coefficient and has the dimension of force per unit of velocity [16]. This parameter is the most difficult to estimate; the other parameters - such as inertia and stiffnesscan be calculated easily or measured statically. [17]

A good way to describe the damping behavior of a system is by using the damping ratio $\zeta$. This is the ratio of the damping coefficient with respect to the coefficient of the same system critically damped. A critically damped system is a system in which no oscillation can occur independent of the magnitude of excitation. The damping coefficient for a critically damped system can be calculated using the following expression.

$$
c=2 \sqrt{I_{m} k}
$$

Where $I$ is the inertia of the body and $k$ is the stiffness of the connecting spring. Thus, a critically damped system has a damping ratio of 1 .

\section{Logarithmic Descent}

When dynamic testing is possible, a good way to approximate the damping ratio is by using a logarithmic descent (Figure 29). This is defined as the natural logarithm of the ratio of two successive amplitudes. It can be obtained from the following expression [17].

$$
\delta=\ln \frac{x(t)}{x(t+P)}
$$

Where $P$ is the period and $x$ is the amplitude of the oscillation. Substituting the characteristic roots for an under-damped system into the previous equation and performing some manipulation results in the following expression.

$$
\delta=\frac{2 \pi \zeta}{\sqrt{1-\zeta^{2}}}
$$


Solving for $\zeta$ yields the following.

$$
\zeta=\frac{\delta}{\sqrt{4 \pi^{2}-\delta^{2}}}
$$

A plot of $x$ versus $t$ generally contains some measurement error. For this reason the previously described method is modified to measure two peaks $n$ cycles apart. The sequential peak values can be denoted by $B_{1}, B_{2}$, etc.

Then

$$
\ln \left(\frac{B_{1}}{B_{2}} \frac{B_{2}}{B_{3}} \frac{B_{3}}{B_{4}} \cdots \frac{B_{n}}{B_{n+1}}\right)=\ln \left(\frac{B_{1}}{B_{n+1}}\right)
$$

or

$$
\ln \frac{B_{1}}{B_{2}}+\ln \frac{B_{2}}{B_{3}}+\ln \frac{B_{3}}{B_{4}} \cdots \ln \frac{B_{n}}{B_{n+1}}=\ln \frac{B_{1}}{B_{n+1}}
$$

thus

$$
\delta+\delta+\delta+\cdots \delta=n \delta=\ln \frac{B_{n}}{B_{n+1}}
$$

or

$$
\delta=\frac{1}{n} \ln \frac{B_{n}}{B_{n+1}}
$$

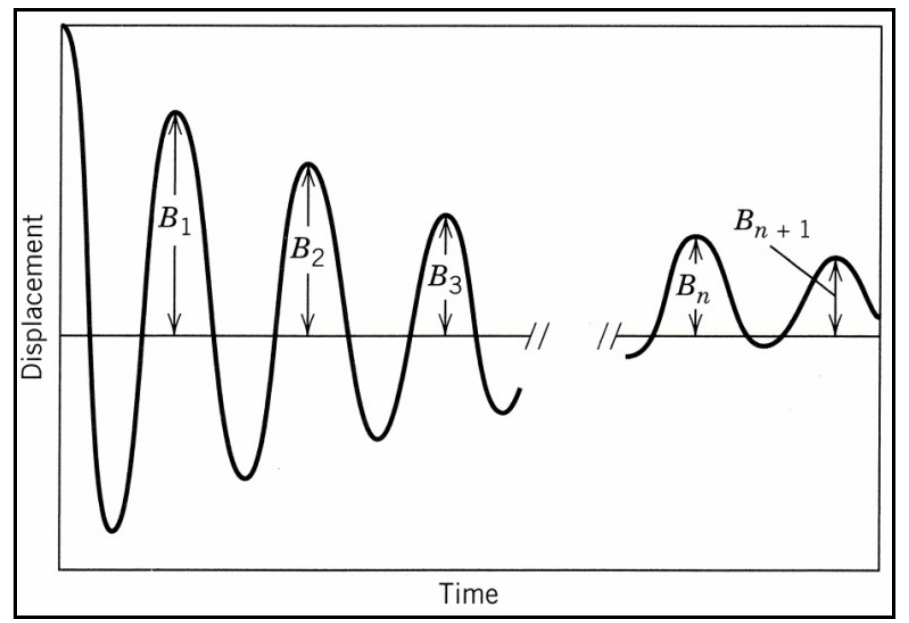

Figure 29. Logarithmic reduction [17] 
Equation 21 can be used to find the damping ratio, and the damping coefficient can be calculated using the following expression.

$$
c=2 \zeta \sqrt{I k}
$$

\section{Gear Mesh Simplified Stiffness}

A simplified method for calculating the stiffness of the gear mesh is to assume that the gear tooth behaves like a cantilever beam or cantilever plate, this approach is suggested by the AGMA standard [31]. This is assuming that its profile is constant along the tooth. The maximum deflection of a cantilever beam can be calculated using the following expression.

$$
y_{\text {max }}=\frac{P l^{3}}{3 E I_{a}}
$$

Where $P$ is the applied load at the tip of the beam, $l$ is the length of the beam (in this case the height of the tooth), $E$ is the modulus of elasticity, and $I_{a}$ is the area moment of inertia (in this case it is assumed to be a rectangular cross section). The area moment of inertia for a rectangular cross section can be determined using the following expression.

$$
I_{a}=\frac{b h^{3}}{12}
$$

Where $b$ is the width of the cross-sectional area of the beam (in this case the operational face width of the gear tooth), and $h$ is the height of the cross-sectional area (in this case the tooth thickness).

Generally, the gear mesh stiffness is calculated from the angular deflection of the gear per unit of torque. This means that the calculated tooth deflection has to be changed to angular deflection. Because the linear beam theory assumes that there is no change in length of the beam, and given that the angle of deformation is small, the overall angular deflection of the gear (see Figure 30) can be calculated using simple trigonometry. 


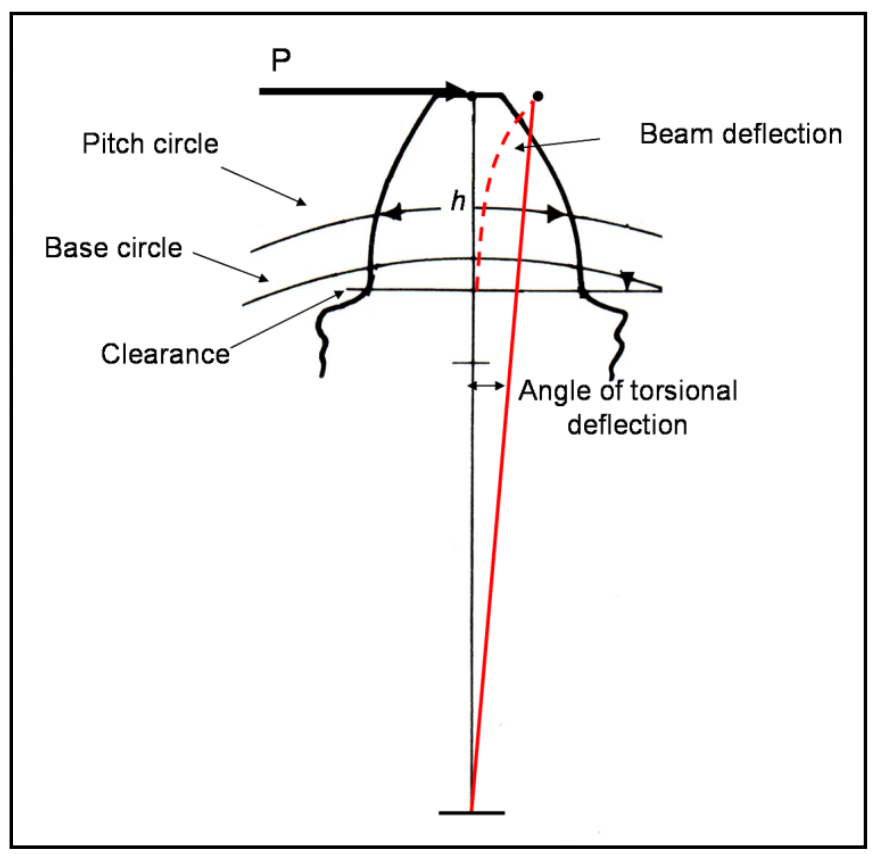

Figure 30. Gear tooth deflection

\section{Mesh Stiffness Calculation Input Parameters}

The beam length is taken to be the sum of the addendum and the dedendum. This assumes that the load is applied at the furthest point from the root of the tooth. The clearance should be added to the dedendum because it increases the height of the tooth. If the specific tooth dimensions are not available, the AGMA standard, full-depth gear tooth specification can be used. The specification describes a relationship between the module and the other tooth parameters [6].

The width of the beam is taken to be the effective face width or the smallest face width of two meshing gears in a gear set. The thickness of the idealized beam can be related to the tooth thickness which also can be obtained from the AGMA full-depth gear tooth specification.

\section{Blade Inertia}

For the blade's moment of inertia the additive property is employed. This states that for a rigid body consisting of $N$ point masses $m_{i}$ with a distance $r_{i}$ to the axis of rotation, the total moment of inertia equals the sum of the inertias of the point masses. It can be expressed by the equation below.

$$
I_{m}=\sum_{i=1}^{N} m_{i} \cdot r_{i}^{2}
$$

To perform this equation the blade must be converted into point masses. This is done by subdividing the blade along it length, calculating the mass for each section, and assuming that the mass is effective at the center of the subdivided section. Figure 31 shows the discretization of a blade into mass-point form. 


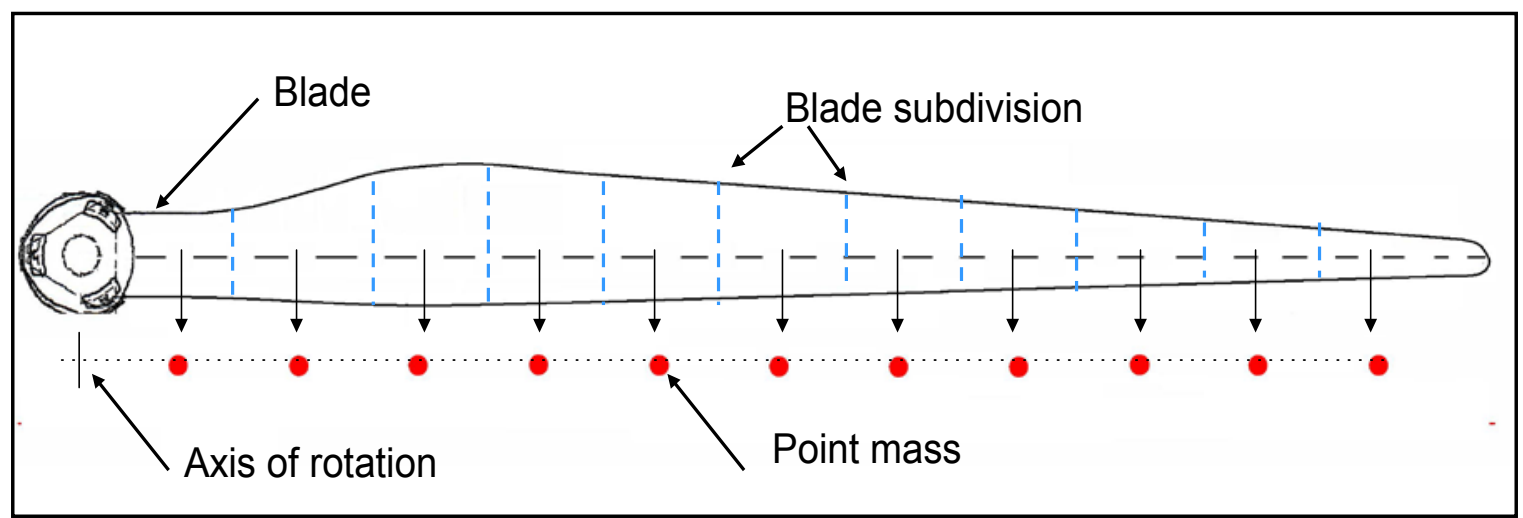

Figure 31. Blade mass discretization

This approach assumes that the blade behaves like a completely rigid body, which might not be a true assumption for certain load cases.

\section{Effective Inertia and Stiffness}

When dealing with gear systems and drivetrains that are composed of a group of independent bodies, each independent body has its own respective inertia and stiffness. A system to simplify the calculations is to adjust the values of inertia and stiffness to an overall effective set of properties.

Depending on the gear ratio, a new relation for the stiffness can be derived from the principle of potential energy. To rescale the inertias to a single lumped inertia, the principle of kinetic energy can be applied.

Figure 32 (below) is a graphic representation of a simple gear system. It is assumed that the shafts have no inertial properties and so only their stiffness is considered. The gear ratio of the system is given by the following.

$$
n / 1
$$

Hence it is $n$. [5] 


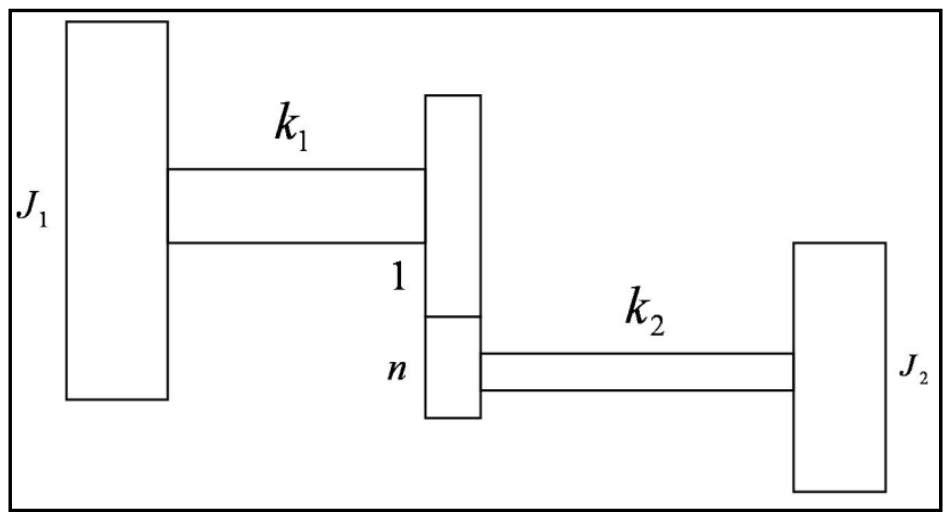

Figure 32. Two-stage representative drivetrain [5]

In Figure 32, representative two-stage drivetrain inertias are represented by $J$ and stiffness of the shaft is represented by $k$. [5]

When the system is compounded the shafts are represented by a single shaft, and a new effective stiffness is calculated by scaling the original values of stiffness with the square of the gear ratio. In the same manner, the inertial behavior of the system also is adjusted by scaling it with the square of the gear ratio [5]. Figure 33 is a graphic representation for the equivalent model of the drivetrain.

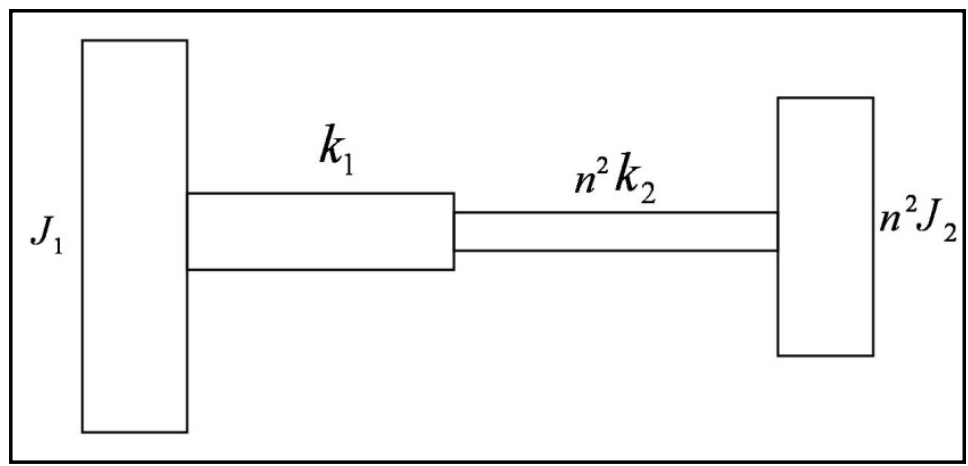

Figure 33. Equivalent one-stage drivetrain with single stiffness and inertia [5]

The reduced stiffness can be calculated by combining stiffness with the following expression.

$$
k_{e q}=\frac{n^{2} k_{1} k_{2}}{k_{1}+n^{2} k_{2}}
$$

Figure 34 shows the graphical representation of the equivalent stiffness. 


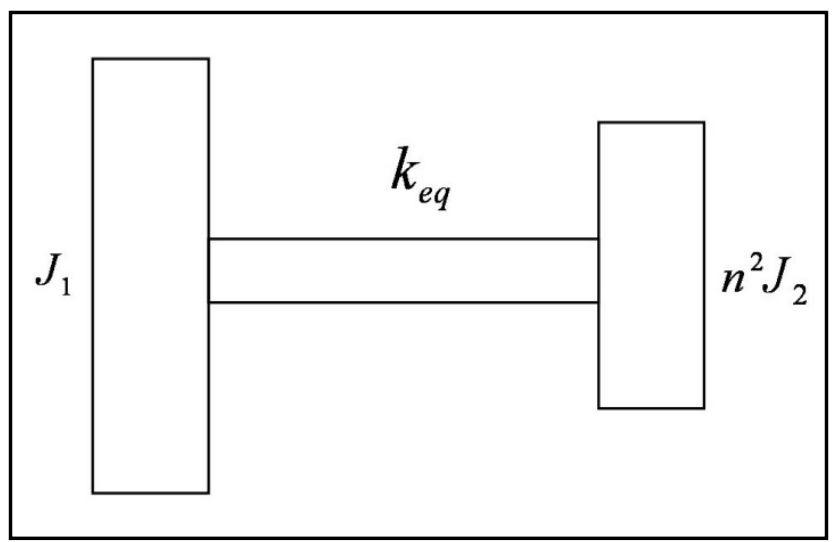

Figure 34. Graphic representation of equivalent stiffness

\section{Progressive Stage Description}

\section{Turbine Description}

To create the baseline model, a physical turbine with a number of particular characteristics was chosen. The turbine selected is a turbine representative of the current industry standard. This turbine also served as a basis for the creation of the simplified models, and their validation by means of data acquisition.

The turbine is a horizontal axis, three-bladed machine with rated power in the range of $750 \mathrm{~kW}$. This is the smallest turbine that can maintain the overall configuration characteristics of larger turbines (1.5 MW and $5 \mathrm{MW}$ ). The turbine has a rotor diameter size of $48 \mathrm{~m}$ and a tower height of $55 \mathrm{~m}$.

The drivetrain follows the prominent modular configuration previously mentioned, and is composed of a low-speed shaft, gearbox, brake, high-speed shaft, and generator. The gearbox follows the typical configuration of a single planetary stage and two consecutive parallel-axis stages with helical gears. The location of the brake on the high-speed shaft allows the isolation of the behavior of the low-speed shaft and gearbox with respect to the generator and the rest of the turbine. The generator used is a double induction generator having the capability of switching to a different number of poles, thus allowing its operation at two separate constant speeds. The simulation of this transition is outside the scope of this report.

The turbine is an upwind stall-controlled type. This configuration eliminates a number of control variables that could greatly influence the loading condition of the drivetrain, due to the high sensitivity to the parameters involved in pitch-regulated control systems.

The physical presence of the turbine and the cooperation of wind-turbine site owners also are valuable components, because they simplify the data-acquisition process.

\section{Stage 1. Simplified Complete Drivetrain Model}

In the first stage of modeling, the entire drivetrain was modeled in a very simple form. The system is composed of two rigid bodies, the first represents the rotor of the turbine and the second represents the generator. These two bodies and their respective torsional inertias are connected to each other with a torsional spring damper joint. With respect to the generator inertia, the influence 
of the gear ratio of the drivetrain is included by calculating an effective inertia that is proportional to the square of the gear ratio (see Effective Inertia and Stiffness, above).

The bodies are connected to the reference frame with only one degree of freedom (rotation), giving the overall system only one DOF per body. This connection has unknown properties such as stiffness and damping coefficients, and is assumed to be without mass. These parameters are determined by the use of experimental data.

Although this is the simplest model that was built, it is of greatest importance because it serves as the basis for the input and validation of the subsequent stages. Additionally, this simplified drivetrain model is used by many aeroelastic codes which are used to generate the loading conditions of the drivetrain. This configuration serves as further validation for the aeroelastic models that already have been validated.

The system with the topology shown in Figure 35 (see Figure 36) was implemented for the semistatic validation. Note that the body representing the generator is constrained to the reference frame with zero degrees of freedom. This simulates the rigid application of the brake on the high-speed shaft. In this configuration the interaction of the generator can be ignored and omitted from the equations of motion.

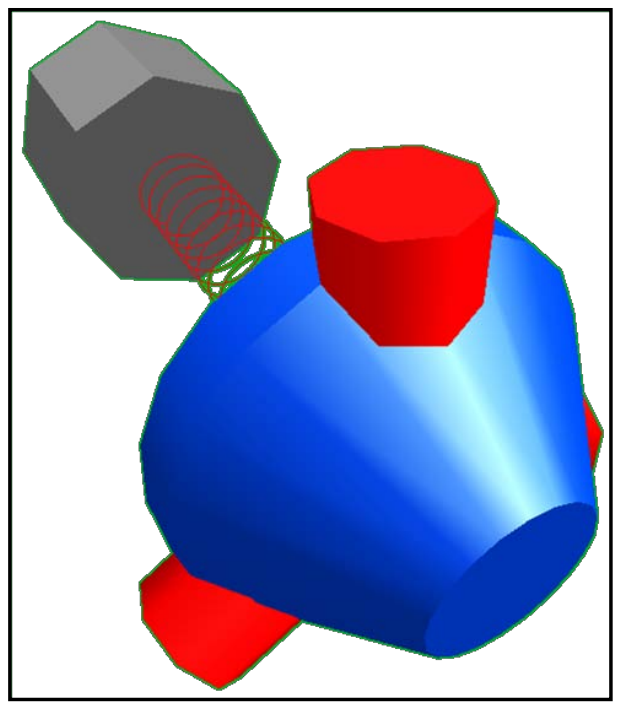

Figure 35. Stage 1 graphical representation 


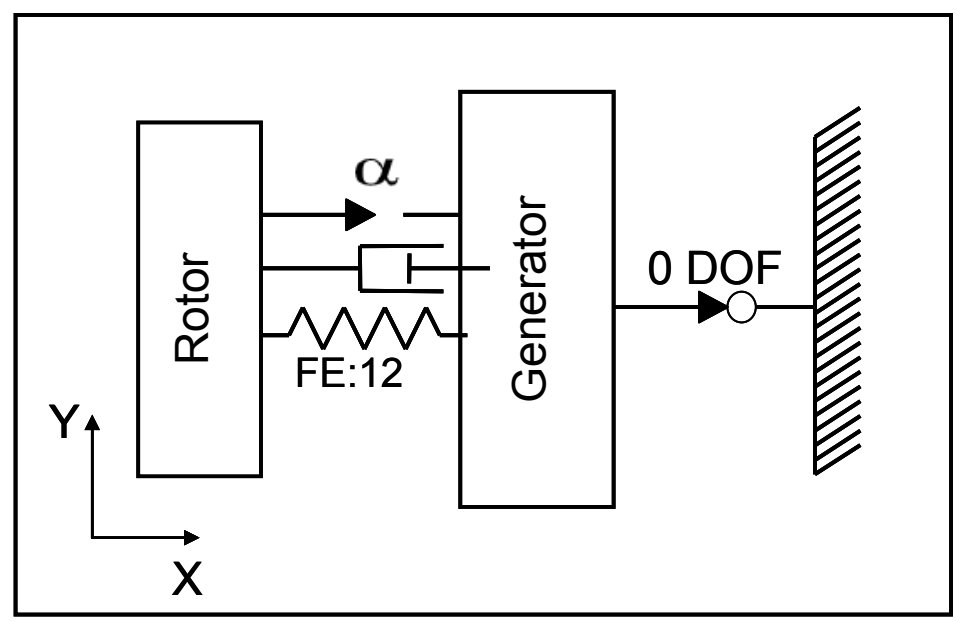

Figure 36. Topology of constrained model

As shown in Figure 37, the constraints imposed on the generator are removed and only rotation around the $\mathrm{X}$-axis is allowed. This system has two degrees of freedom, therefore the behavior of both main components must be monitored to obtain the correct dynamical behavior of the connecting spring.

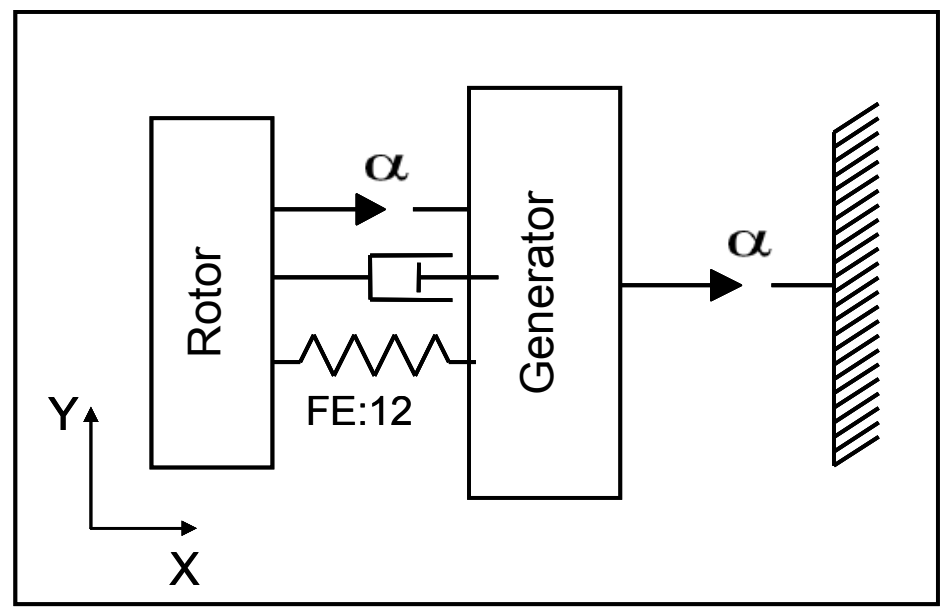

Figure 37. Topology of unconstrained model

\section{Data Acquisition and Validation}

The validation of the semi-static model in Figure 38 seeks to determine a reasonable agreement of the experimental response and the simulated response. In this case, experimental data was collected from the field from the existing and operational $750-\mathrm{kW}$ stall-controlled turbine (described above).

The first step of the validation involved the acquisition of the physical parameters of the system. For this simple model, the only requirements were the mass and moment of inertia of the rotor. 
The bulk masses for the blade and hub were acquired, [27] and a typical mass distribution for a $750-\mathrm{kW}$ blade was scaled linearly to meet the bulk mass of the blade. The mass distribution also was scaled to match the actual length of the blade. Using the specific characteristics of the mass properties, the blade was discretized into point masses along its length. The mass moment of inertia for each point mass was calculated and summed to obtain the overall inertia of the blade. This approach assumes that the rotor is entirely rigid, and neglects the effects of the flexibility of the blades. The model has only one degree of freedom, therefore the only inertia of interest is the rotation around the axis of the rotor (see Blade Inertia (above) for more detail).

To obtain the stiffness and damping, acceleration measurements were recorded on the turbine itself. These measurements reveal the overall frequency of the drivetrain as well as the rate of damping of the system. The data collection consisted of performing mechanical braking maneuvers to induce excitation to the drivetrain. The normal braking procedure - the deployment of blade tips - was overridden, and the mechanical brake was applied manually (see the drivetrain section (above) for a description of normal braking maneuver). When the mechanical brake is applied, the entire drivetrain undergoes torsional loads to oppose the inertial forces of the rotor. Consequently, the drivetrain coils and uncoils like a spring. The desired information from the system can be interpolated from this response.

Acceleration was measured on the gearbox housing because it is joined to the bedplate by elastomers and a stronger excitation signal was captured than if it had been collected on a component rigidly attached to the main frame. The data analyzed was collected on the top of the gearbox housing because it provided a horizontal flat surface where accelerometers could be easily mounted. To enhance the signal collected, the accelerometers were located at the point farthest from the axis of rotation of the input shaft. The acceleration data was collected by uniaxial accelerometers placed in the vertical direction to prevent tower sway movement from being recorded in the data sets. The data collected was scaled so that the acceleration information collected away from the axis of rotation subsequently could be translated into angular acceleration, and could be compared directly to the results of the dynamical simulation.

No existing experimental data having the same parameters as the data collected was found. Therefore no comparison with any existing validated data could be made. The response expected from the collected data should have resembled the behavior observed in the final stages of specified braking maneuvers, which can be found in AGMA standard 6006. This existing braking maneuver data, however, depicts torque behavior with respect to time and does not use acceleration as a parameter. 


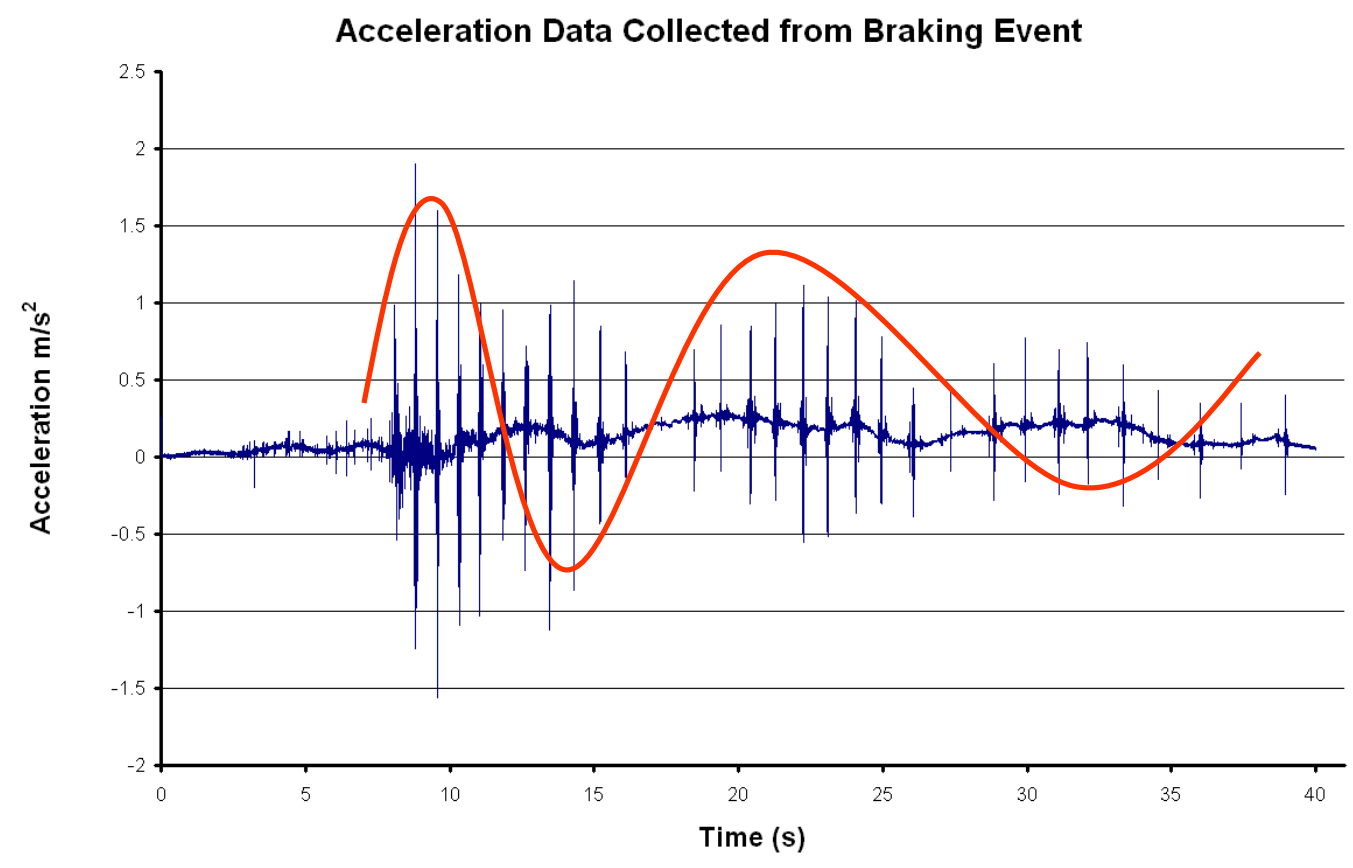

Figure 38. Experimental data and secondary frequency response

The data collected nevertheless reveals the overall frequency of the system, because it is a parameter that is easy to capture. In the case of the damping the amplitude of the excitation presents a secondary frequency, which can be seen in the figure above as a red line and which raises doubt with respect to the validity of the data. Regardless of the precision of the data, this report depicts a method to validate the progressive models generated. Moreover, the values obtained from the validation of the models correlated quite well with the theoretical calculated values. This leads to the conclusion that the approach followed and the data obtained were correct.

The acceleration recorded is characterized by strong point excitations that diverge in great magnitude with respect to the mean acceleration value. These slender excitations contain a single data point for each peak regardless of the high frequency $(1,000 \mathrm{~Hz})$ of data collection.

Consequently, a minute discrepancy at the time of the excitation and the data collection creates a change in the recorded response. This can be seen as a much lower frequency influencing the magnitude of the excitation. This phenomenon is produced by a small offset in the frequency of the system and the data-collection frequency. In Figure 38 this frequency is shown as a red line.

To elaborate on this phenomenon, a pair of graphs is presented in Figure 39. The graph on the left depicts an arbitrary sinusoidal wave. The vertical lines represent the point in time in which the data is collected. The red dots mark the point where the sinusoidal response crosses the data collection; therefore, the dots depict the magnitude of the data collected for that point in time. If the data points collected were joined, then the response obtained would be that described by the red line in the graph on the right side of Figure 39. It shows that a secondary frequency bounds the amplitude of the recorded response. This secondary frequency is depicted by the green line in the right-hand graph. This essentially is the same phenomenon as seen in the experimental data collected, although in that case it is produced by the short period of excitation. 


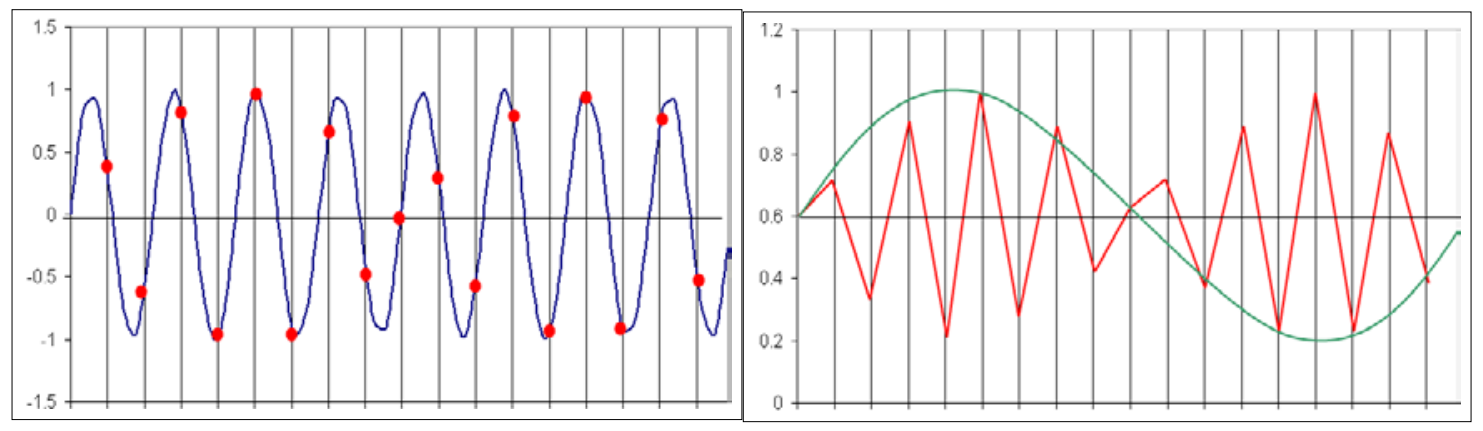

Figure 39. True response (left) and collected response (right)

The effect of nonlinear behavior can be seen in the experimental data, because the initial frequency recorded is not the same as that at the end of the data collection. This is due to the stronger influence of clearances with respect to smaller amplitudes of excitation. The slight changes in frequency consequently alter the secondary frequency observed.

The gaps between the excitations reflect the time in which the internal clearances of the gearbox such as backlash and bearing are absorbed. This occurs for every cycle, because the loading is fully reversed once the system has been excited.

From the data presented above, the frequency of the periodic peak excitations was recorded as $1.1 \mathrm{~Hz}$. This value was used in combination with the previously mentioned rotor inertia to calculate a simplified rotational vibrating spring mass system. Using the assumption that the system behaves as a free vibrating system, a theoretical stiffness of $6.25 \mathrm{E}+07 \mathrm{Rad} / \mathrm{Nm}$ was calculated and input into the Stage 1 model shown in Figure 34 (see Torsional Free Vibration; Torsional Free Damped Vibration). The simulation was performed and the frequency obtained by the simulation had good concurrence with the experimental response (see Figure 40).

The reduction in the magnitude of the excitation is a consequence of the damping of the system. Due to the particular characteristics of the data recorded a trend curve could not be fit. This is because the points of interest are the greatest in magnitude of the secondary frequency and there are not enough points to result in a converging curve.

The reproduction of the secondary frequency was achieved by changing the time integration characteristics of the simulation; more specifically, by increasing the size of the integration time step. This produced a response similar to the point excitation and the secondary frequency observed in the field, although the shape of the simulated curve resembles a smooth sinusoidal wave.

A theoretical damping coefficient was calculated by assuming that the overall system behaved as a free damped vibrating system. The result is a simulated response that closely follows the field data recorded. 


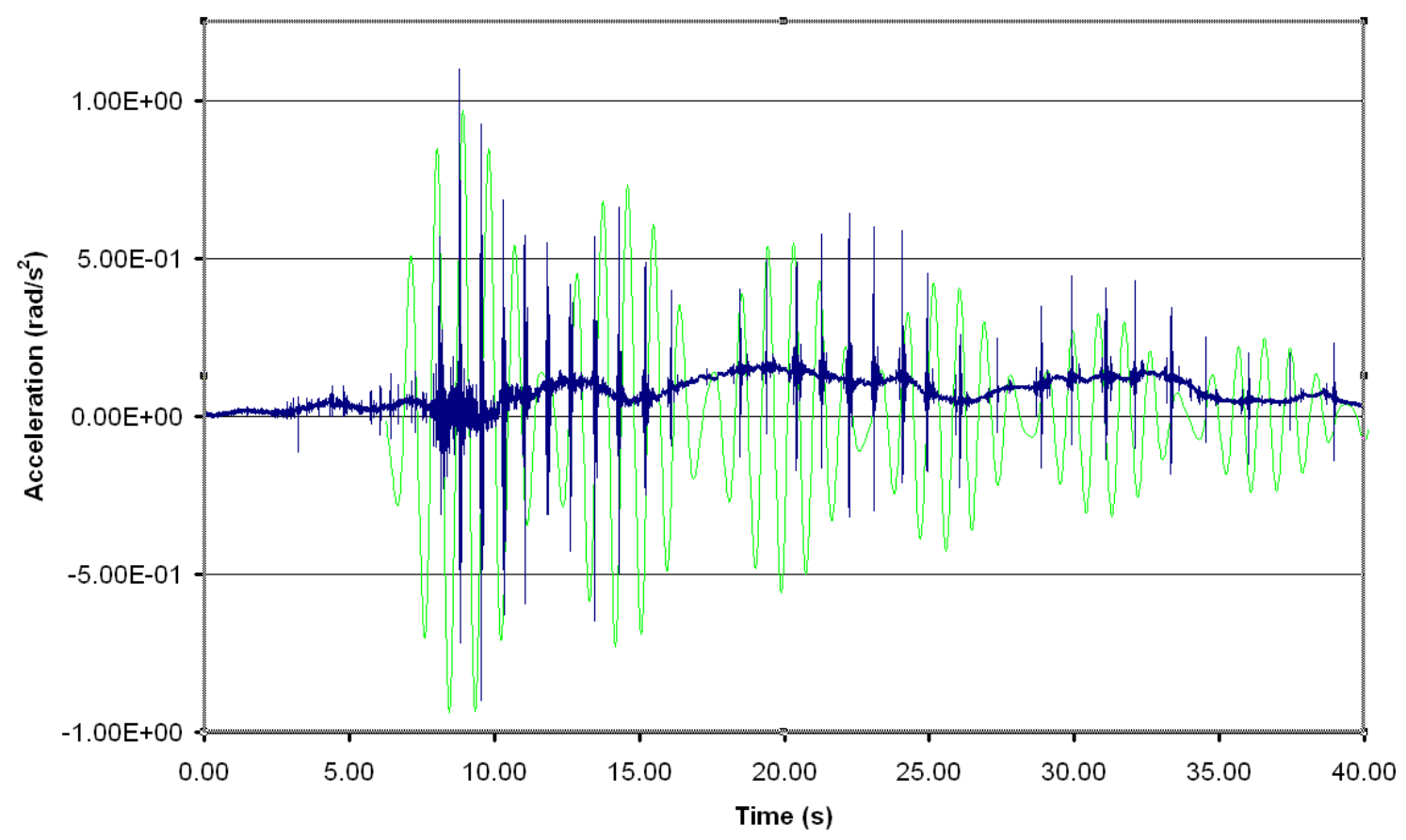

Figure 40. Experimental data and simulated response

When the damping and the stiffness were tuned to match the recorded data, the simulation time step was increased to obtain the representative form of the oscillating system. The values obtained for the stiffness and damping are used as guidelines for the validation of the rest the progressive models. Figure 41 depicts the simulated response with the refined time step. This case uses a time step of 50 steps/sec.

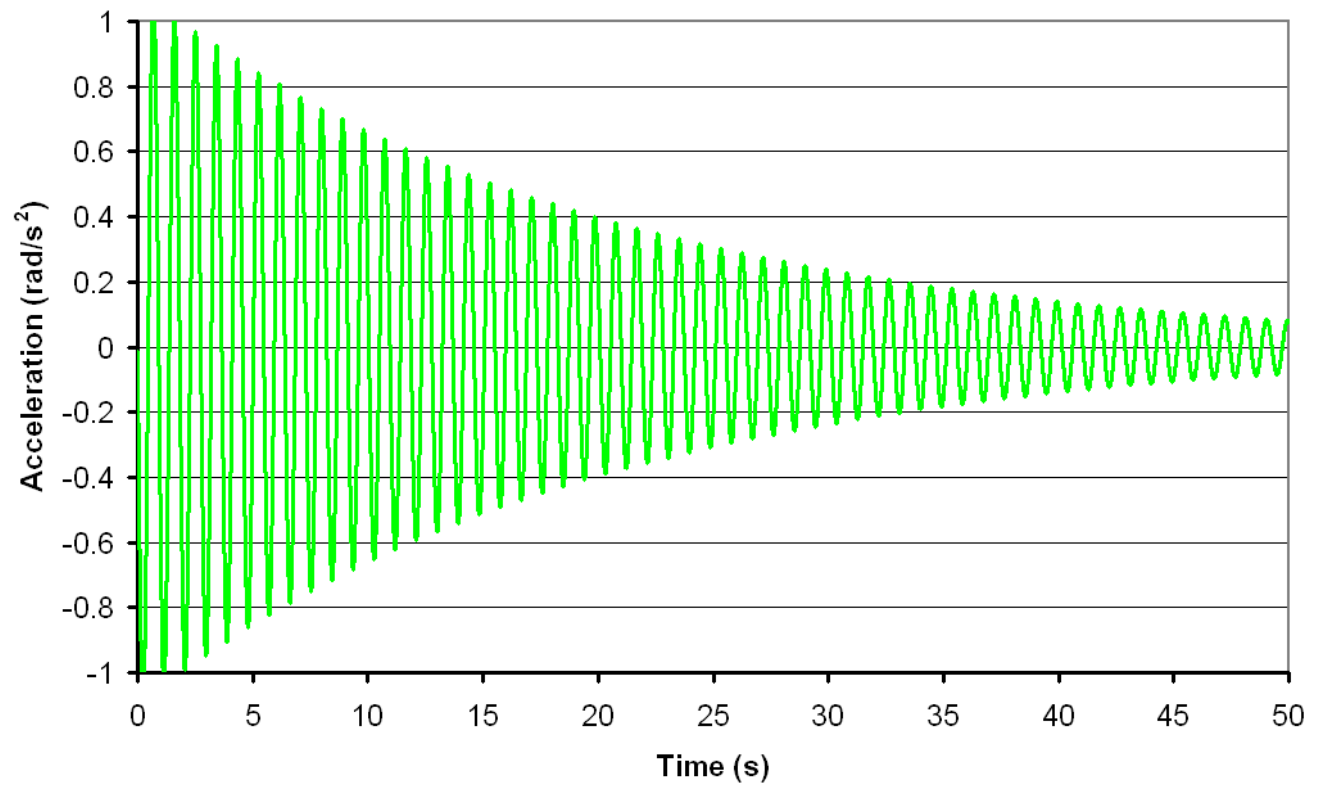

Figure 41. Stage 1 simulated response with refined integration time step 


\section{Stage 2. Simplified Rotor and Generator with Multiple-Stage Gearboxes}

Stage 2 (see Figure 42) implements the same approach for the rotor and the generator as used in Stage 1. Every major component of the gearbox was modeled, however, such as gears and shafts. The system has one DOF for each rotating body.

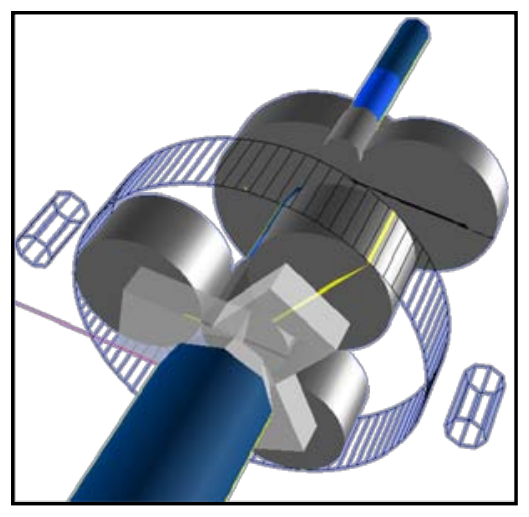

Figure 42. Stage 2 graphical representation

This approach accounts for the torsional compliances resulting from the bending and contact deflection of the gear teeth, as well as torsional deflections of the shafts. The model ignores the added torsional compliance from the bending of shafts and from bearing deflection [10].

Spring dampers joining both gears were used to simulate gear interaction. These joints lie over the line of action and are subjected to the tangential forces of the gears [19]. The overall torsional response of the system was obtained, as well as the response from the internal components in a dynamical and coupled manner. The SIMPACK force element FE:14 was used as the tangential spring interaction [21].

The gear bodies were represented by rigid primitives used in the 3-dimensional visualization, and do not have any influence in the calculation's outcome. The shafts were simulated by torsional spring dampers or FE:12 in SIMPACK, giving the insight of the torsional shaft deflection as a separate parameter. All the respective torsional inertias from each individual component must be calculated from the masses and geometries as inputs for the model. It is assumed that there is no change in direction, and that the connecting springs between gears always will be under tension (no backlash).

Figure 43 shows the topology of the planetary stage of the gearbox, which is the most complex stage. One of the requirements of the force element SIMPACK FE:14 is that the input and output shaft-force elements are defined with respect to an arbitrary common body such as the gearbox housing. This limits the ability to simulate the planets' inertial behavior. To remedy this situation a massless connecting body was created. This body has the dynamical outputs such as torque and angular position of the planets. To account for the inertial behavior, three planets with respective masses and torsional inertias were created and attached to the planet carrier, with only one allowable rotation. Using this configuration, the rotational behavior of the planet on the planet carrier is taken into consideration. To transmit the rotational behavior of each planet to the 
massless body, the rotational degrees of freedom were constrained; more specifically, degrees of freedom in alpha were constrained.

Figure 43 is a graphic representation of the planetary stage. Due to the limitation of the SIMPACK FE:14, each gear interaction requires the definition of one housing (red box) and two SIMPACK FE:14 elements. The first housing represents the planet ring interaction with the respective gear ratio, as well as the added rotation from the trajectory of the planet. The input shaft is represented by the planet carrier and the output shaft is represented the by the massless connecting body 1 . The mass and inertias of the massless body are assigned by the constraint of rotation in alpha. The importance of this setup is that the masses and positions of the planets influence the inertia of the planet carrier.

On the second housing the planet-sun interaction is represented and, naturally, the sun-planet gear ratio is implemented to define the force element. The torque and angular velocity of the input shaft of this section are transferred by a zero DOF constraint between both massless bodies.

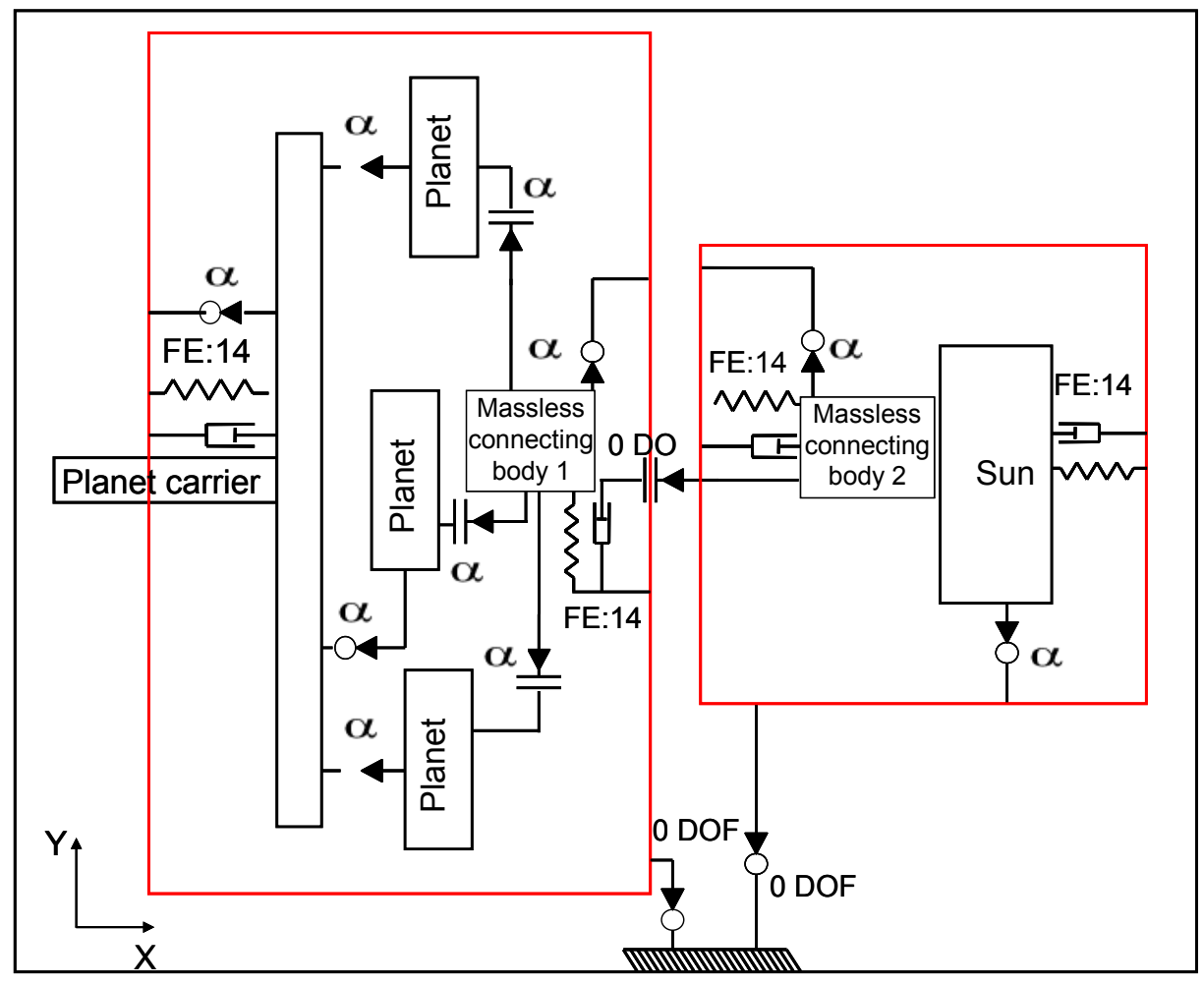

Figure 43. Planetary stage topology

Figure 44 is a continuation of Figure 43, expanding the topology to the remaining internal stages of the gearbox. At the far left, a portion of the sun is represented as a continuation of Figure 44; although, to avoid overcrowding, the SIMPACK force element FE:14 is not represented. This figure shows connecting shafts for the different internal stages of the gearbox. Each individual shaft is represented by two independent bodies. The first section of the shaft is connected to the previous gear by a 0 DOF connection. The two sections of the shaft are connected with a single- 
axis rotating joint. The state quantities of the joint are used by the SIMPACK FE:12 (torsional spring) to calculate forces and displacements of the second section of the shaft. The two helical stages are represented in the same form as the planet-sun interaction.

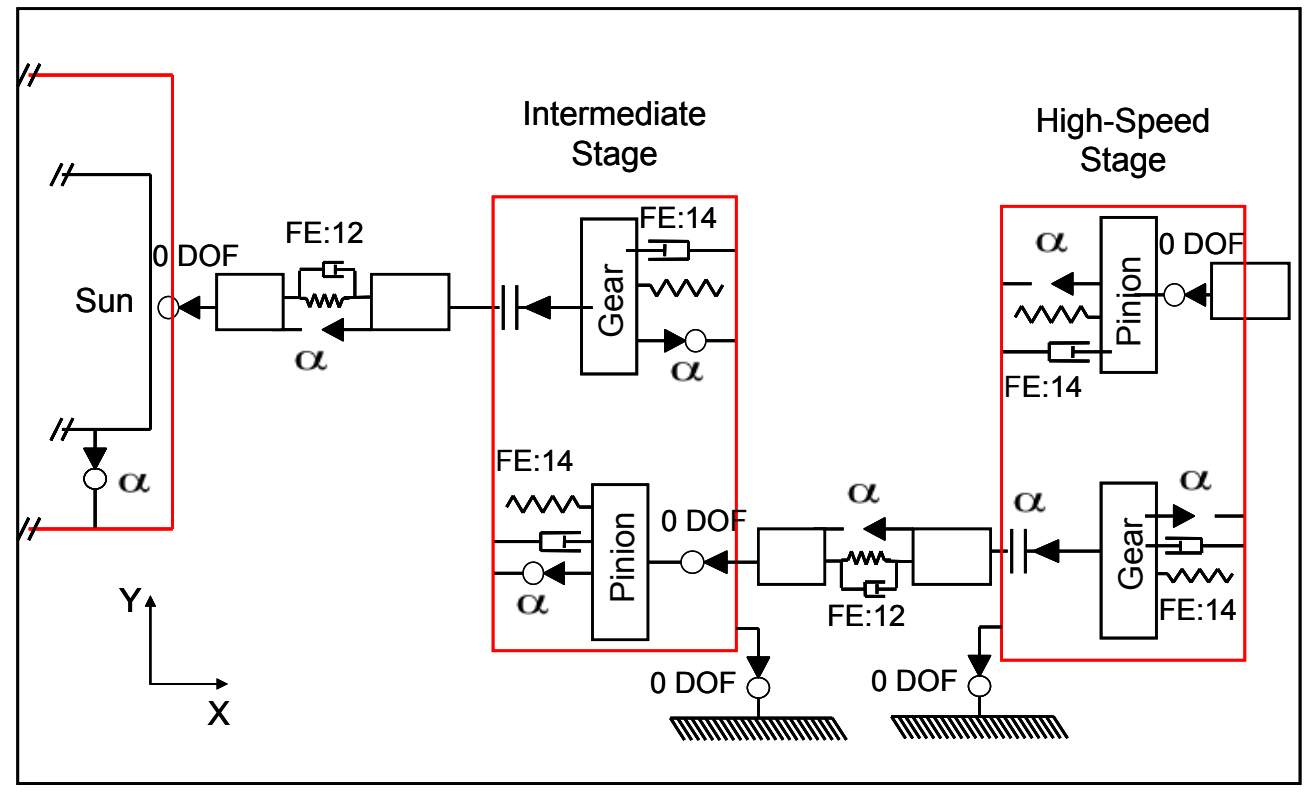

Figure 44. Subsequent gearbox stages topology

As noted, all internal components are simulated in this stage. This calls for the dynamical properties of each component. The following sections explain how specific parameters required for this model were acquired.

\section{Mesh Stiffness}

The parameters of the spring dampers connecting each gear wheel or element FE:14 were substituted by the gear mesh stiffness. The gear mesh stiffness was calculated as a single simply supported beam, and it was assumed that the thickness of the beam remained constant and unchanged by the tooth profile. The stiffness of the tooth therefore is constant along the tooth. The length of the beam was taken to be the height of the tooth including the respective clearance. This approach assumes the most conservative scenario, because it only takes into account one gear tooth being in contact. It also assumes that the contact occurs at the farthest point of the line of action, instead of acting at the pitch diameter.

The force applied to the tooth for the stiffness calculation is the tangential force of the gear pair, which is tangent to the pitch diameter and perpendicular to the tooth. This approach does not account for multi-tooth contact or for changes in the contacting number of teeth; hence the stiffness remains constant and linear. The model also neglects to consider the existence of backlash.

\section{Shaft Stiffness}

The shafts were modeled as ideal steel circular beams. The influence of steps and stress risers was neglected, therefore an average or representative diameter had to be considered to be able to 
generalize the behavior of particular sections of the shaft. It was assumed that there was no torsional deflection on the shaft under the section braced by gears. The entire defection of the shaft occurs on the internal distance between the gear pairs; this distance is called the effective length. This can result in fairly high stiffness because in some situations the effective length of the shaft or the section undergoing torsion can be quite short. This assumption ignores the relatively large clearances that characterize key and spline connections between gears and shafts. These clearances can result in a nonlinear load deflection characteristic. [10] The shafts were subdivided into two equal sections, represented by two independent bodies connected by the spring element having the calculated effective stiffness.

\section{Inertias}

Inertias were calculated from the physical properties of the gearbox components. The rotor inertia was calculated in the same manner as was done in Stage 1. The shafts were assumed to be solid cylinders and all shafts were subdivided into two equal but separate sections. The inertias were calculated for each independent section of the shaft. The shafts were joined by a single DOF joint, and the overall inertia and interaction of the shaft was calculated by the simulation code. The inertia for the respective gears was calculated by assuming that the gears behaved as a solid cylinder described by the pitch diameter and the face width of the respective gear wheel.

\section{Data Acquisition and Validation}

The calculated theoretical values for the shaft and gear mesh stiffness were input into the model, the simulations were performed, and the results were compared to the accelerometer data collected from the field. Both frequencies were compared, and the simulated frequency that was obtained was higher than that for the experimental data. A proportional adjustment of the shaft stiffness was performed to match the experimental data collected. This required that the stiffness be reduced by $10 \%$ for each individual shaft, thus matching the $1.1 \mathrm{~Hz}$ frequency of the empirical data.

To validate the damping values, a more quantitative approach than that performed on the Stage 1 model was pursued. This approach assumed that the overall system behaved as a single degree of freedom, torsional free, damped, vibrating system. Using equation 22 (see Logarithmic Descent, above) the damping coefficient of each individual shaft can be calculated. This equation is dependent on the stiffness and mass or inertia properties of the single degree of freedom system, thus a reduction of the actual degree of freedom of the system was required. This was achieved by assuming that the shaft for which the damping coefficient was to be calculated was the only section of the system allowed to move. Therefore all the other components of the system were assumed to be infinitely stiff.

For the inertia component an effective inertia had to be calculated due to the effects of the gear ratio (see Effective Inertia and Stiffness, above). Again, the approach of using the conservation of energy was applied to the system, although - in this case - instead of increasing the stiffness of the shaft, the inertia of the rotor was reduced. This is because the stiffness that is input into the simulation code must be the representative stiffness of the shaft, regardless of the gear ratio. The damping calculated therefore must be correlated to this stiffness. The effects of the gear ratio with respect to stiffness and damping are calculated by the software. This approach was used iteratively for each subsequent shaft; therefore the damping was calculated depending on the stiffness of the shaft as well as the position along the gearbox. 
The quantity missing — but which can satisfy the equation - is the damping ratio. This was taken to be the damping ratio from the experimental data, which also was calculated from a logarithmic descent.

The same braking event as that used in the model for Stage 1 also was induced on the more complex Stage 2 model. The damping ratio was calculated from the response obtained and then compared with the damping ratio of the experimental response. The difference was minimized by scaling the damping coefficients of the simulation, with a 5\% scaling reduction of the calculated theoretical value. This produced an error percentage of less than $3 \%$ for the damping ratios.

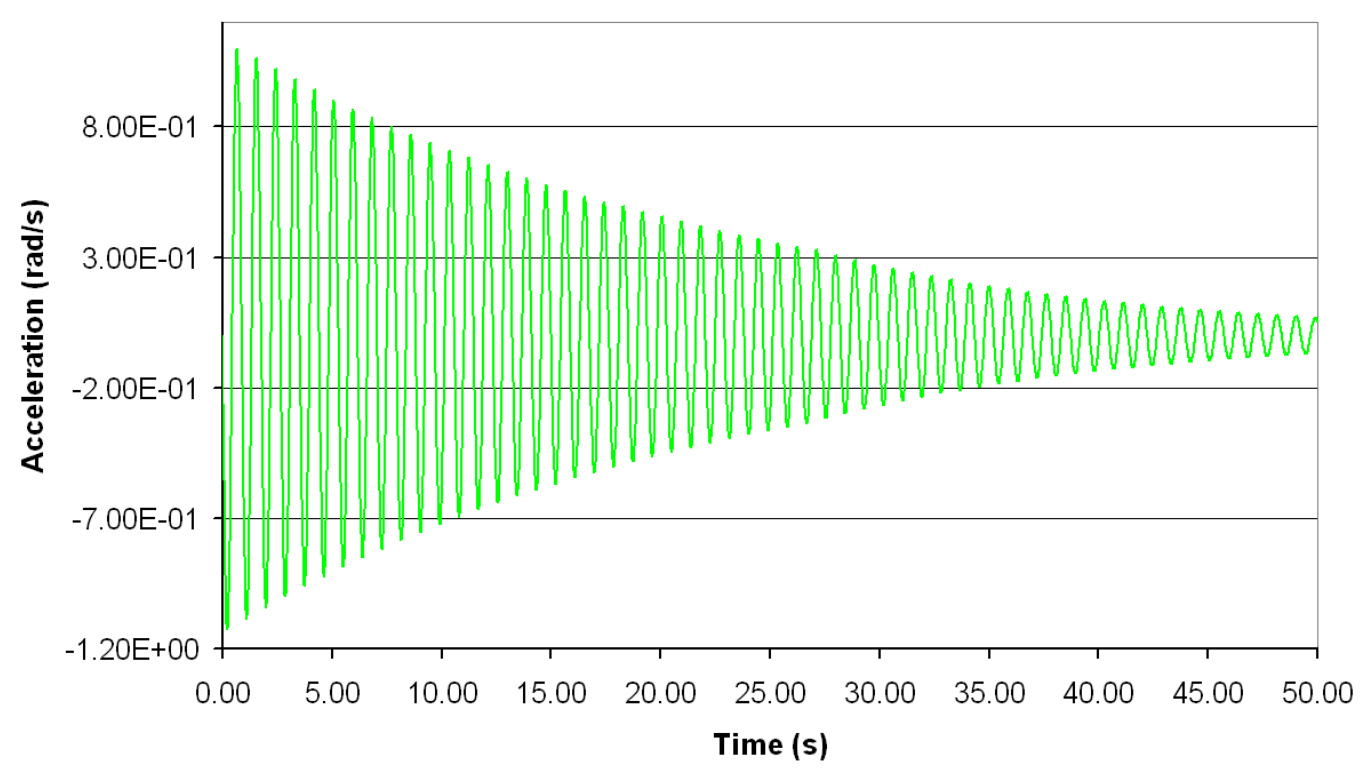

Figure 45. Stage 2 model simulated response with fine integration time step

Figure 45 depicts the response from the Stage 2 model. A fine integration step of $50 \mathrm{steps} / \mathrm{sec}$ also was used to obtain the response. Notice that, although the percent error between Stage 2 and the data collected is very small, visual differences in the damping envelope can be seen.

\section{Stage 3. Multiple-Stage Gearboxes with Contact Element Implementation}

Stage 3 (see Figure 46) recreated the physical representation of Stage 2, excluding the rigid bodies for the gears. The primitives were created from standard gear-wheel parameters. These parameters were utilized to create contact elements capable of reproducing multi-tooth contact, backlash, and changes in the direction of the gear rotation. The shafts are represented as torsional spring dampers (as was the case for the previous stage). Additionally, the connection between the gears and the housing has only one DOF. 


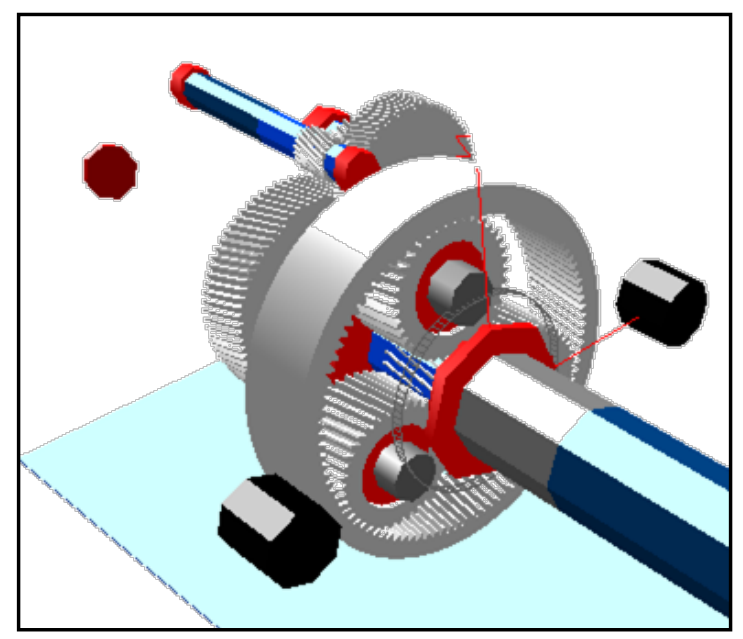

Figure 46. Stage 3 graphical representation

Stage 3 uses a built-in SIMPACK force element FE:225. The only requirement of the force element is that a body-fixed marker in the center of each gear be defined. This marker is required to establish the reaction forces between the two interacting gears [22].

To account for the force interaction of all components, the ring gear also must be considered. The Stage 3 ring gear was fixed to the gearbox housing with zero DOF. The force interactions of the system included planet to ring as well as planet to sun, and the two forces and their directions were considered in the analysis. The direction and magnitude of the forces-especially those of the planets - are of great importance because the force on the tooth changes direction with each cycle, which increases the fatigue damage. The SIMPACK force element FE: 225 can account for changes in the gear center distance and backlash [22]. For this model, because the joints of the system have only one degree of freedom, the center distances do not change; nevertheless, backlash is included.

In Figure 47 the topology of the planetary section is represented for Stage 3 of the analysis. The main difference between Stage 2 and Stage 3 is the implementation of the new SIMPACK force element FE:225. This element eliminates the need for the multiple housing representations; one common housing is used for the entire gearbox model. Note that the only interaction between the planets and the ring gear, and the planets with the sun, is the force element FE:225. This eliminates the need to implement the two massless connection bodies shown in Stage 2. The planets have only one degree of freedom with respect to the planet carrier, and there are no added constraints to the system. The simulation with the new force element, as well as with all the gear components, provides a much more realistic representation of the system and its interactions. 


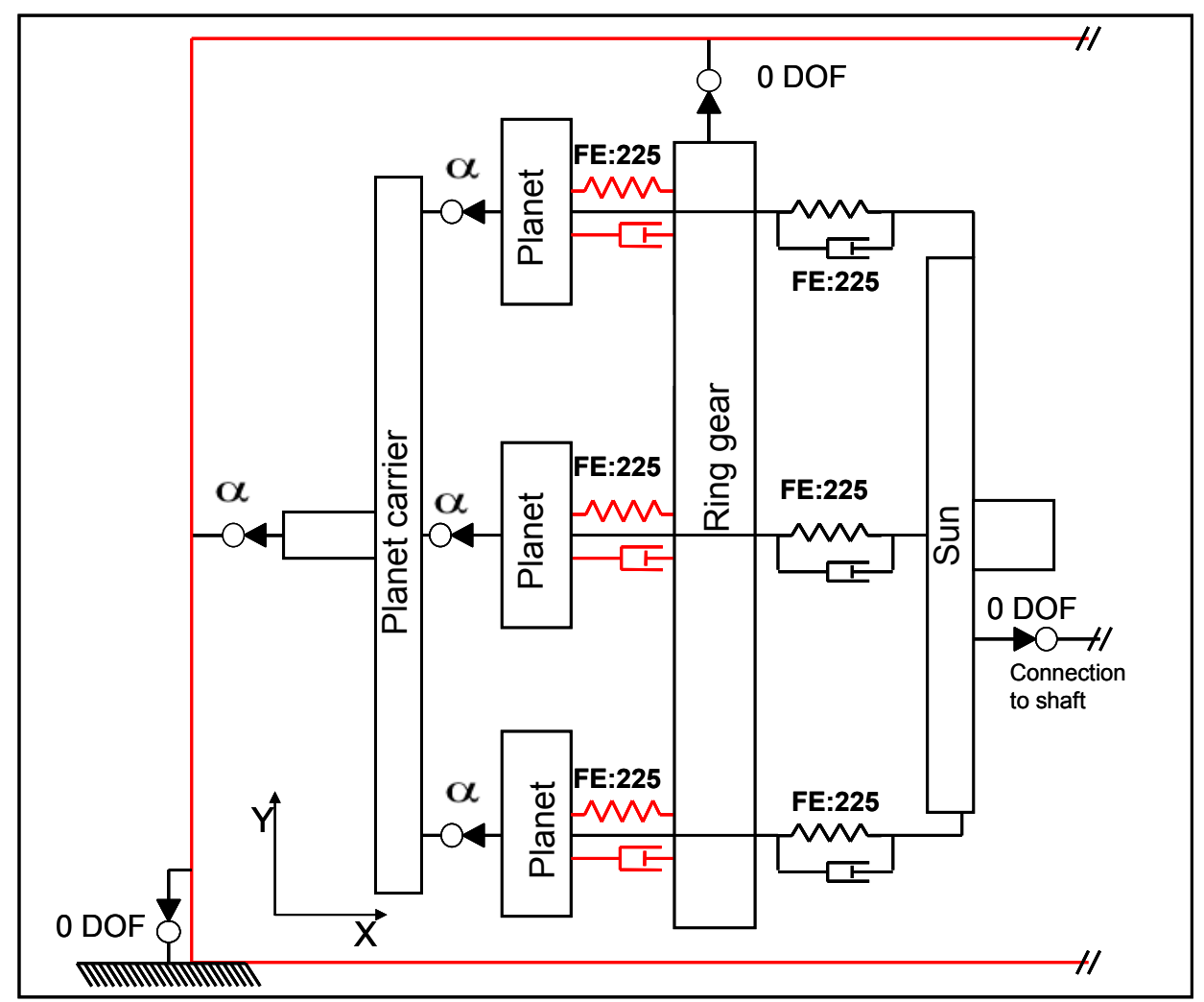

Figure 47. Topology of planetary stage with FE:225

Figure 48 is a continuation of Figure 47, expanding the topology to the remaining internal stages of the gearbox. The shafts are represented in the same manner as in Stage 2, with the use of SIMPACK FE:12 as a torsional spring damper. The main difference is that the shafts now share a common housing with a joint that only allows rotation in alpha. The two helical stages also can be seen. These are connected to the nearest shaft by a zero DOF joint and SIMPACK force element FE:225 is between them.

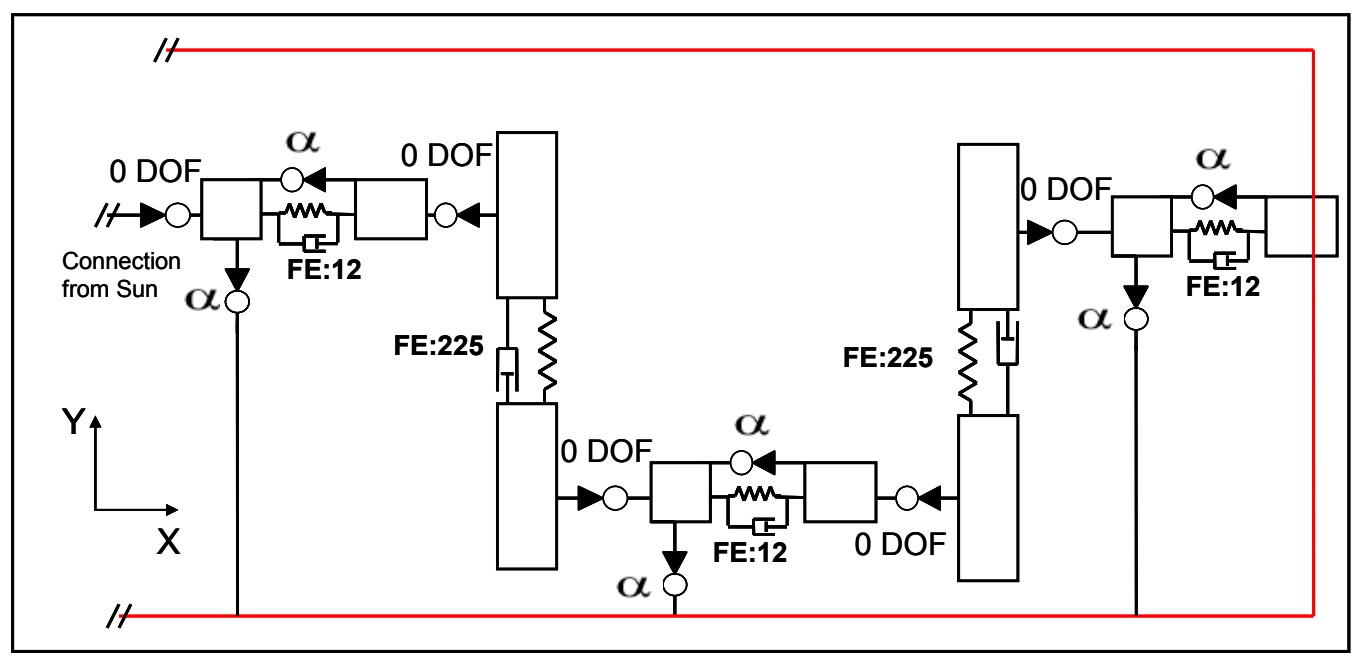

Figure 48. Topology of subsequent gearbox stages with FE:225 
Although Stage 3 is more complex it still is extremely similar to Stage 2, making for a good comparison between stages. The following sections explain the acquiring of the specific parameters required for this model.

\section{Mesh Stiffness}

In this case, the mesh stiffness is calculated in a more accurate manner internally by the simulation code. The software calculates mesh stiffness using the parameters describing the gearwheel primitive, together with additional user-supplied material properties. As noted above, the advantage of using the SIMPACK force element FE: 225 is that the system accounts for multi-tooth contact and the variation in stiffness resulting from the change in number of teeth. This model also accounts for backlash (see SIMPACK Force Element FE:225, above).

\section{Shaft Stiffness}

As in the previous model, the shafts for Stage 3 were modeled as ideal steel circular beams. The influence of steps and stress risers was neglected. Therefore, to generalize the behavior of particular sections of the shaft, an average or representative diameter was considered. For more information, see the Shaft Stiffness section for Stage 2 (above).

\section{Inertias}

Inertias were calculated from the physical properties of the gearbox components in the same manner as was done in the Stage 2 model. The main difference in the inertial calculation is that, in the previous model, all shafts were subdivided into two equal, separate sections. In this model, due to the more detailed configuration, the shaft was not necessarily subdivided into equal sections. This resulted in the need for recalculation of the inertia for the particular sections.

\section{Data Acquisition and Validation}

The theoretical parameters were input into the model, simulations were performed, and the results were compared to the accelerometer data collected from the field. The frequencies of both results were compared and, in this case, the simulated frequency was less than in the experimental data. A proportional adjustment of the shaft stiffness was performed to match the data collected - the stiffness was increased by $10 \%$ for each individual shaft, thus matching the frequency from the empirical data. This is necessary due to the effect of backlash; the stiffness of the gear mesh goes to zero in the reversal of direction. The shaft stiffness therefore must be increased to account for this phenomenon.

As was done in the previous model, it was assumed for this model that the overall system behaved as a single degree of freedom, torsional free, damped, vibrating system, and the same approach of the logarithmic descent was performed (for more detail, see the description of the Stage 2 model).

The same braking event that was performed in the Stage 1 model was induced in the Stage 3 model. Using the response obtained, the damping ratio was calculated and compared with the damping ratio of the experimental response in the same manner as for the Stage 2 model. The difference between the experiment and the simulator response was reduced to a minimum by scaling the damping coefficients of the simulation. Using a scaling increment of the calculated theoretical value of $20 \%$ resulted in a less than $1.5 \%$ error for the damping ratios. 


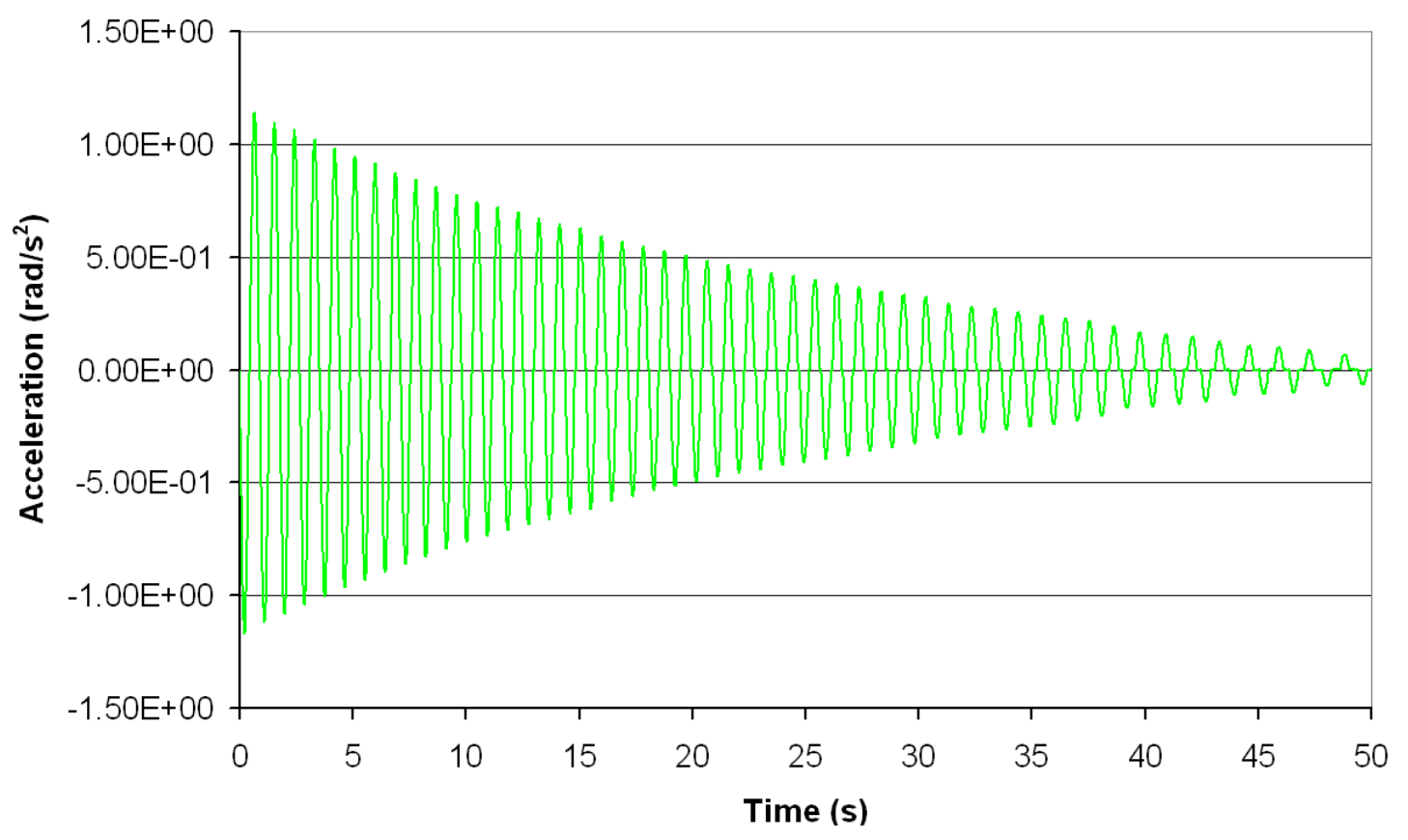

Figure 49. Stage 3 model simulated response with fine integration time step

Figure 49 shows the response of the Stage 3 model. A fine integration step of 50 steps/sec also was used to obtain the response. Note that although the percent error between this stage and the experimental response is very small, noticeable visual differences in the damping envelope appear when it is compared with the previous two stages.

\section{Stage 4. Multiple-Stage Gearboxes with Contact Element Implementation and Bearing Stiffness}

Stage 4 adds three degrees of freedom to the joints between the rotating bodies and the housing of the gearbox. These additional degrees of freedom were constrained using the SIMPACK force element FE-43. This force element can represent six independent stiffness and damping coefficients (three translations and three rotations). Force element FE-43 can implement linear or nonlinear stiffness, which is of great use due to the nonlinear stiffness and clearance that characterize bearing behavior. Although SIMPACK FE-43 is capable of constraining six DOF, only three stiffness and damping coefficients are implemented in this stage (translation $\mathrm{x}, \mathrm{y}, \mathrm{z}$ ). 


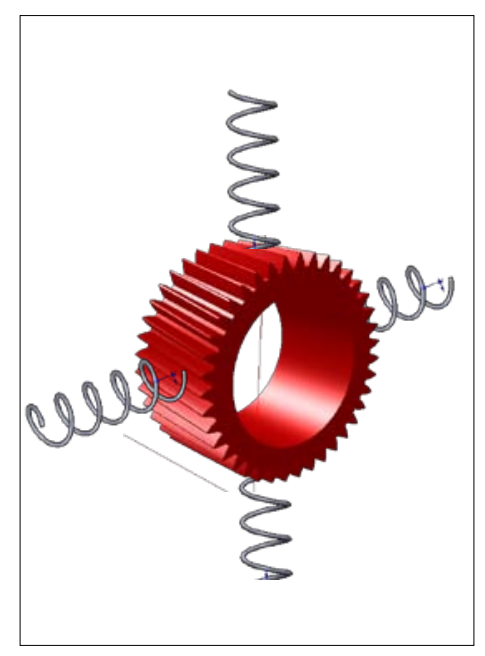

Figure 50. Bearing stiffness representation

The addition of the force element required the adding of new markers that describe the attachment points of the bearings. This is very relevant because the bearings are represented by three stiffnesses (see Figure 50), and each shaft needs a minimum of two constraining forces. This situation required a more precise description of the housing and the exact positions of the bearings. Additionally, the length of the shafts and the position of the bearing on the shaft were of great importance because they had to match the housing to prevent the undesired prestress conditions. This was not required on the other models because they were purely torsional models with rigid bodies.

Constraints also were added to the connection between the input shaft and the gearbox.

Originally only rotation was constrained, here all six DOF are constrained. This simulates the rigid coupling between the shaft and the gearbox, and reveals how nontorsional loads affect the internal components of the system.

The diagram in Figure 51 illustrates the additional degrees of freedom for the planetary stage. Comparing this figure with Figure 47 shows that the overall structure of the model is the same, with the addition of new force elements and new translational degrees freedom per joint. 


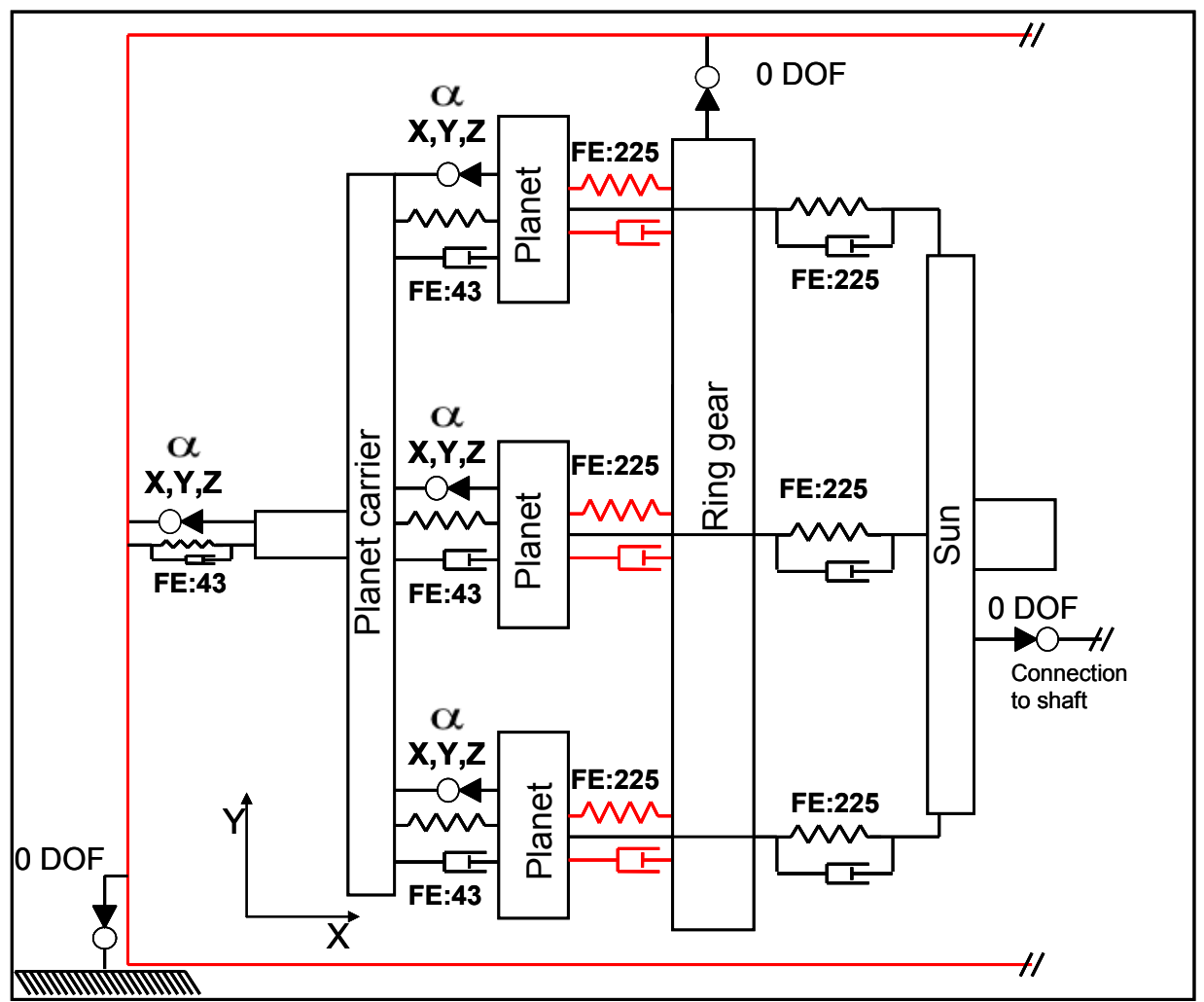

Figure 51. Planetary stage with added degrees of freedoms and force constraints

\section{Special Consideration for Bearings}

Due to the sun bearing's floating characteristic, special attention should be paid to that bearing. Generally, the sun bearing is located outwards from the point of contact of the gear so that the sun can float freely to find its optimal position. This behavior tends to evenly distribute the load from the planets to the sun among the points of contact. To properly simulate the sun interaction its bearing should have six degrees of freedom. In many real-world applications the load distribution is not even, regardless of the floating sun. It would be valuable to monitor the behavior of this load under different loading conditions.

\section{Validation and Parameter Acquisition}

The stiffness and damping coefficient values are the same as for the Stage 3 model; the Stage 4 model simply adds new degrees of freedom to the previous model. The only important parameter to be validated is the bearing stiffness. These stiffness coefficients are highly complex because they have six degrees of freedom, a nonlinear behavior in each direction, as well as different clearances. Although they reveal important information regarding the misalignment of the components, their influence on the torsional compliance is not as noticeable. Therefore the experimental data utilized to validate the previous models is not valuable for the validation of bearings displacements and compliance. Arbitrary values were used for the bearing stiffness, and the relative displacement of components such as the planet carrier was monitored so that the importance of the new degrees of freedom could be observed. 


\section{FAST Model Description}

\section{FAST Input Parameters}

The detailed description of the input parameters for the simulation code FAST_AD required that a number of parameters be interpolated and calculated. The following sections briefly describe the parameter-acquisition process.

\section{Blade Characteristics}

A number of parameters describing the blades were required, such as: length, cord and twist distribution, airfoil characteristics and location along the blade, mass distribution, stiffness distribution, and mode shapes. Due to the lack of some of this information, a similar blade from a turbine of the same power rating was used as a reference blade to aid in the scaling of the factors.

The length as well as cord and twist distribution were measured from an existing blade. The reference blade's airfoil characteristics and respective locations also were used. The overall mass of the blade was obtained from existing documentation. The mass distribution was scaled linearly to meet the overall mass of the simulated blade. The mass distribution also was scaled so that the blade length and the center of mass would match the blade. In case of the stiffness distribution, the same approach of linear scaling was followed.

For the mode shapes calculation, the modal frequency response of the blade was recorded from the field. This was done by manually inducing first and second mode in the flap direction, and first mode in the edge direction. The response was recorded using accelerometers. The frequencies recorded were compared with frequencies from other similar blades for reassurance. The mode shapes for the blades were calculated from the data described.

\section{Tower Properties}

The mass and stiffness parameters were calculated from existing drawings of the towers, which described the tower's overall geometry as well as its wall thickness. The side-to-side frequency response was measured by exciting the structure with a similar mechanical braking maneuver (described in the model development section). The required mode shapes for the model were calculated from this information.

\section{Generator Models}

As noted, the turbine to be simulated has a variable induction generator that allows it to operate at two different constant speeds (see Figure 52). This is achieved by changing the number of poles from six to four for operation at 1,200 rpm and 1,800 rpm, respectively. As stated in the FAST user manual, the simple induction generator model is too simple to be used for a turbine start up. Therefore the Thevenin equivalent for a three-phase induction generator was used. The parameters for this model were taken from a generator with similar characteristics. In the case of the normal operation, the simple induction generator which describes a linear relation between torque and speed was used. The parameters from this simple model were obtained from manufacturer specifications. 


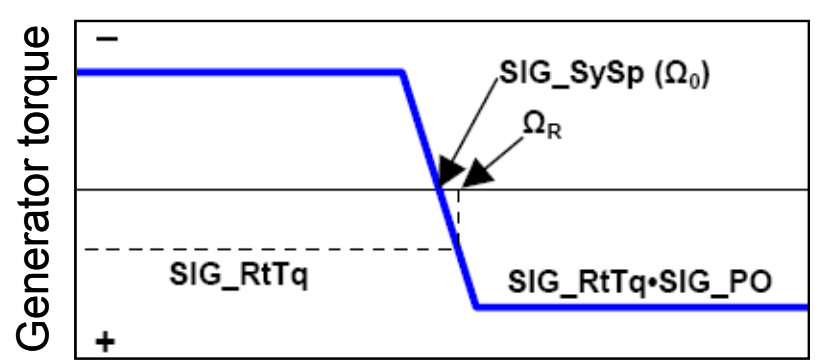

Generator speed

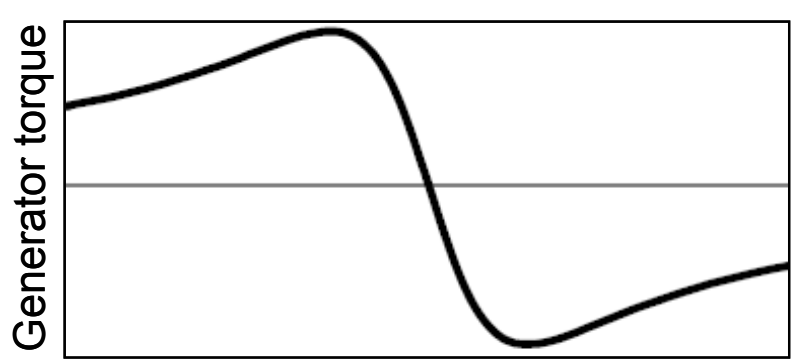

Generator speed

Figure 52. FAST_AD generator models [18]

\section{FAST Generated Load Cases}

A number of load cases to be input into the more detailed drivetrains were generated using the FAST model mentioned above. These load cases included start-up maneuvers at rated wind speed, normal operation at rated wind speed, and emergency breaking maneuvers. The rated wind speed is the speed at which the turbine generates its rated power (in this case $16 \mathrm{~m} / \mathrm{sec}$ ). The load cases were simulated both with constant wind speeds and turbulent conditions.

The emergency braking maneuver consists of a sequence of events in which the generator goes offline - eliminating the applied torque - and the emergency brake is deployed after a short period. An emergency braking maneuver creates one of the harshest loads that the wind turbine system can undergo, due to the high torsional loads and continuous reversals of the loading. Figure 53 is a graphical representation of a braking maneuver, simulated with FAST for the turbine modeled in this report. The event is performed at rated wind speed under high turbulence. The figure also shows the different stages of the braking event.

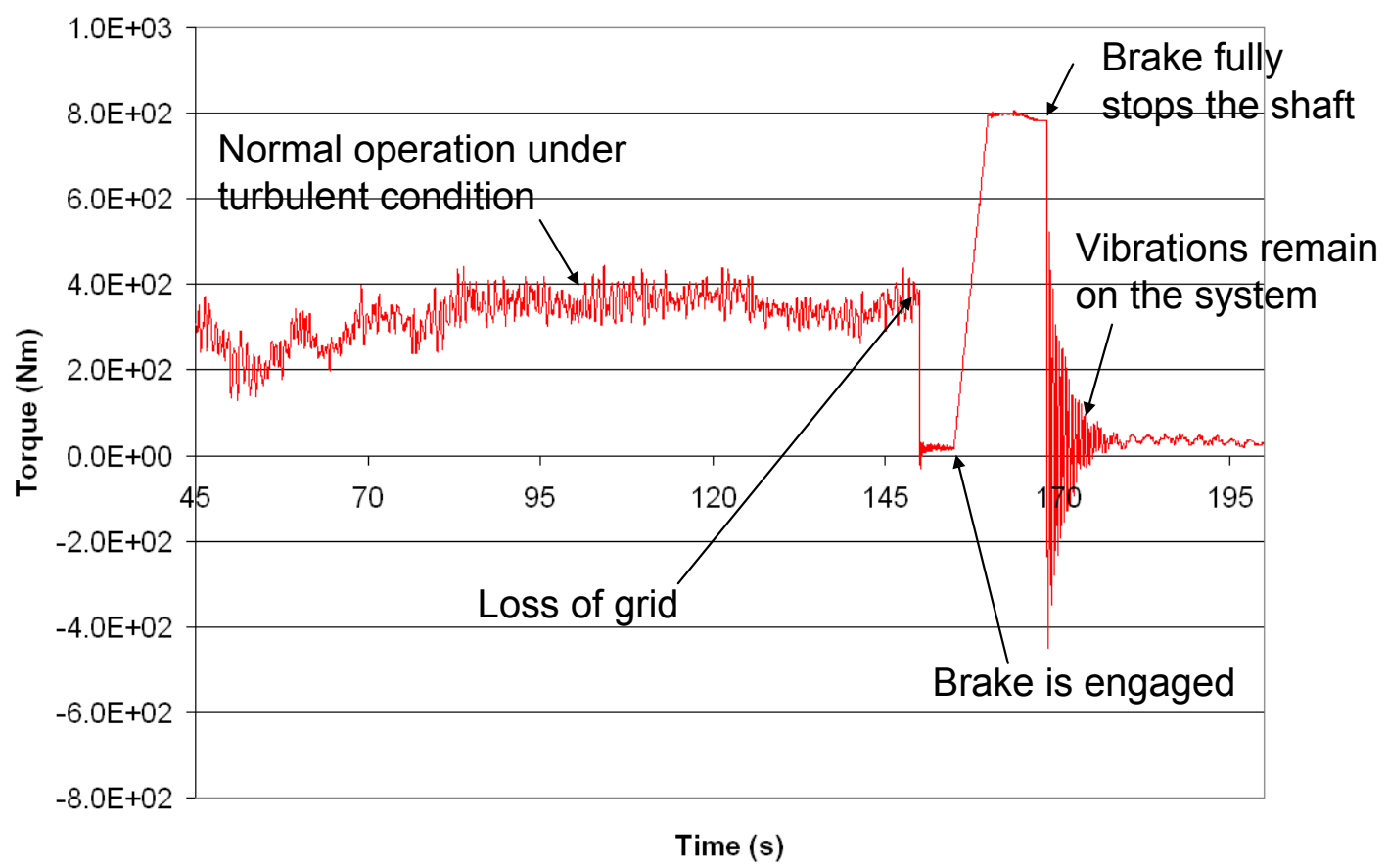

Figure 53. FAST_AD simulated braking maneuver and event description 
The loading conditions were used as an input for the Stage 4 model. This model was chosen because it has the greater number of degrees of freedom and the validity of its output is more tangible. The input load chosen was a braking event from a constant-wind condition at a rated wind speed. The constant wind speed was chosen because of its numerical simplicity; turbulent input files tend to be numerically heavier. The last section of the braking event was chosen due to the fully reversed loading conditions. The aim is to show possible output files that could be used for further study, but which still capture the dynamical scenario.

Figure 54 shows the angular position change of the planet carrier. This is important because changes in the angle of the planet carrier result in poor tooth-load distribution of the gears. Uneven load distribution on gear teeth can result in micropitting, which would progress into macropitting and is a common failure mechanism of gearboxes in the industry. The angular misalignment of the planet carrier also results in uneven load distribution between the planets and the ring. The same happens for the sun-planet interaction if the configuration of the gearbox does not include a floating sun.

Angular Displacement of the Planet Carrier

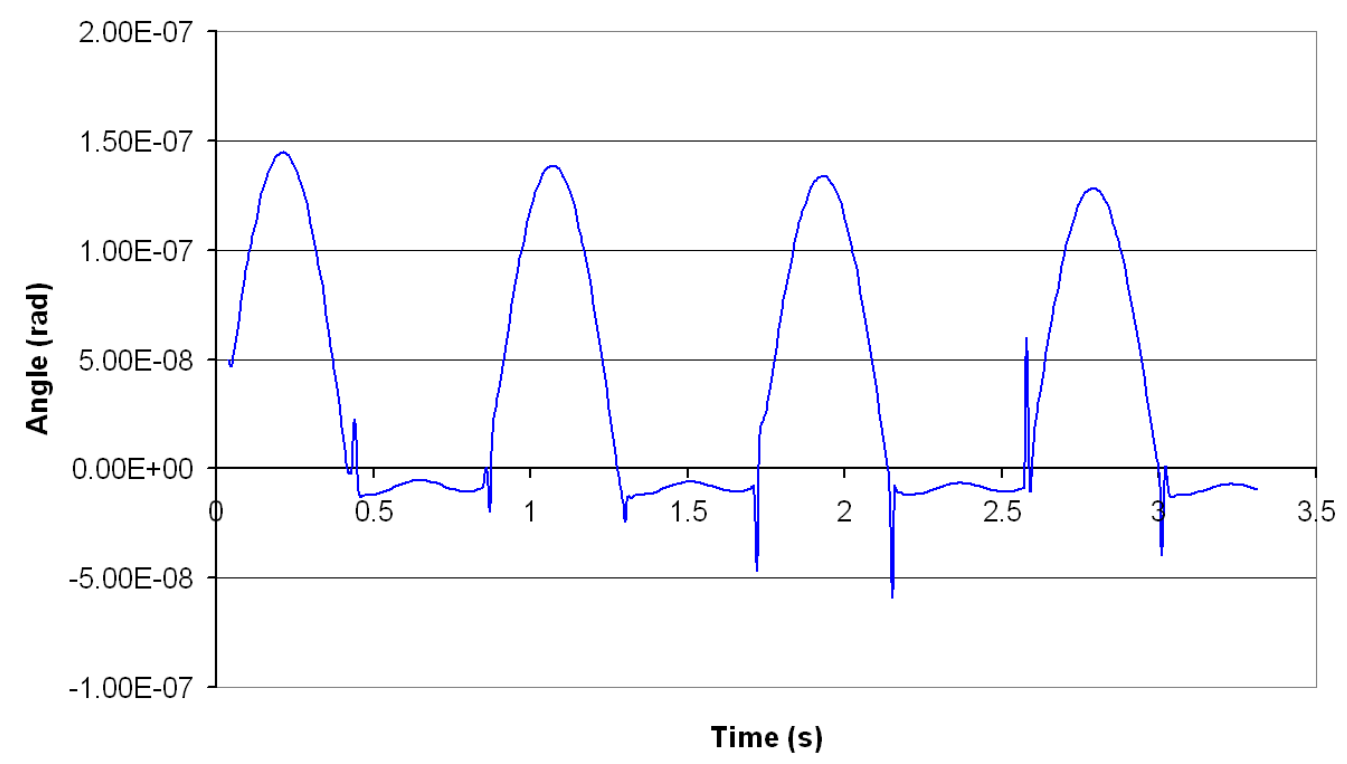

Figure 54. Planet carrier displacement under braking event

Figure 55 depicts axial displacements in the intermediate stage shaft. These deflections are driven by the axial loading generated from the helix angle of the gears. The force generated by the axial loading is a possible reason for bearing failures. Notice the step midway through the oscillation; this is consequence of the effects of backlash. The data collected for this figure was gathered at the final stages of the oscillation so that the influences of the backlash recorded. The backlash interaction is much less noticeable with larger oscillations. It can also be said that the backlash contributes the nonlinear behavior of the stiffness. 


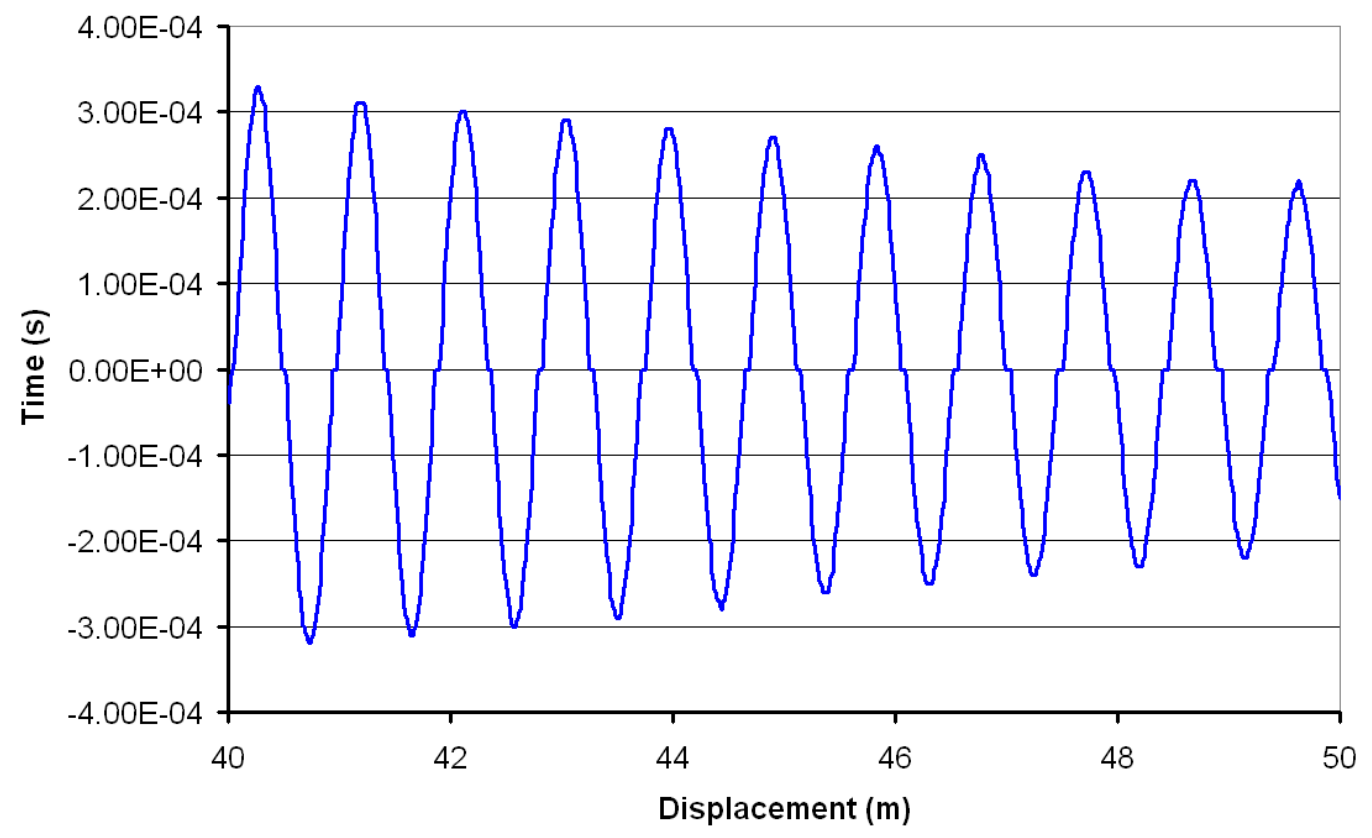

Figure 55. Axial displacement under breaking event

\section{Model Comparison}

This section compares the different models under operational loading conditions. The input load cases implemented were generated by FAST_AD, simulating operation under turbulent conditions at rated wind speed and the simple induction generator model. Models presented here focus only on the gearbox, therefore the interaction of other components such as generator and rotor are not considered. This leads to the implementation of input parameters such as angular velocity and torque to be used in the models. To obtain the dynamical interactions of the internal components of the gearbox, the angular velocities of the rotor were used as input on the model's low-speed shaft. Loads inherent to the high-speed shaft from FAST_AD were applied as an opposing torsional load to the high-speed shaft of the gearbox, which revealed the internal load interaction of the gearbox.

The first parameter compared between the different drivetrain models was the torque at the highspeed shaft (which was the input torque), and the torque at the low-speed shaft (which was the output torque). Figure 56 gives more insight on the previously noted loading condition. 


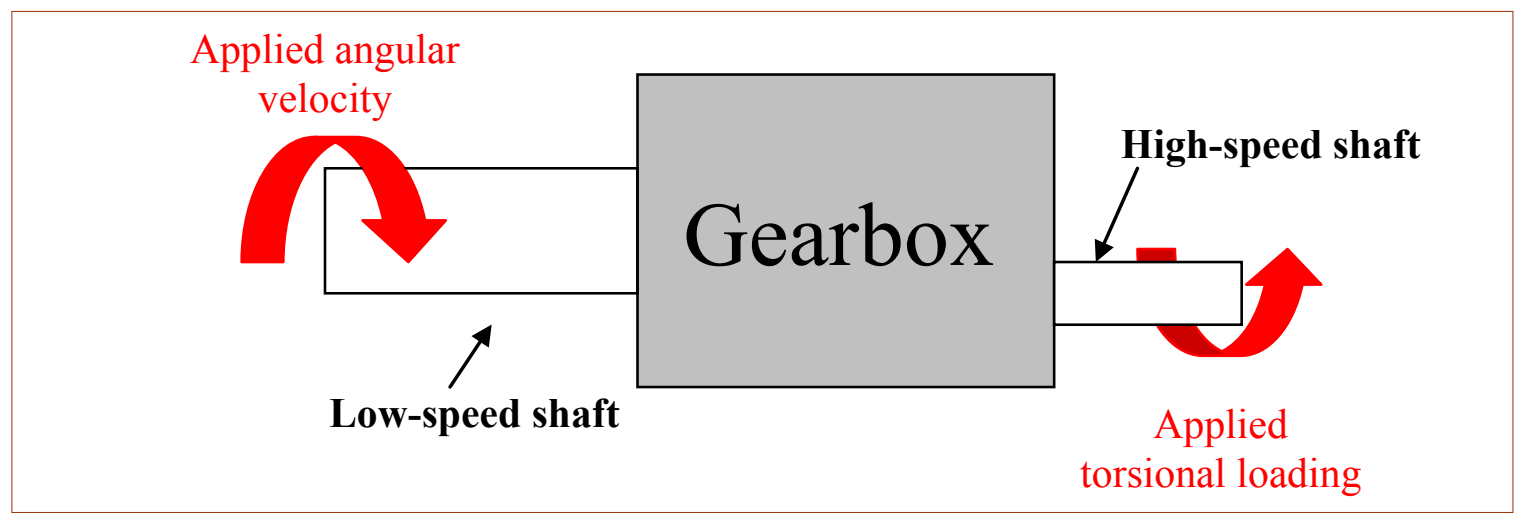

Figure 56. Model comparison loading configuration

The Stage 1 model is the simplest model, and is far too simple to be compared to the other models. It does not have a gear ratio, therefore the magnitude of the output is the same as the input. A simple solution is to adjust the generator by increasing its inertia; this gives a better approximation of the dynamical behavior, although it is still too rudimentary to mimic the true behavior of the gearbox. Additionally, the minute changes in torque cannot be captured by using a single large spring to represent the entire drivetrain. Figure 57 shows the torque relations described above. Notice that the magnitudes are quite similar but have opposing signs.

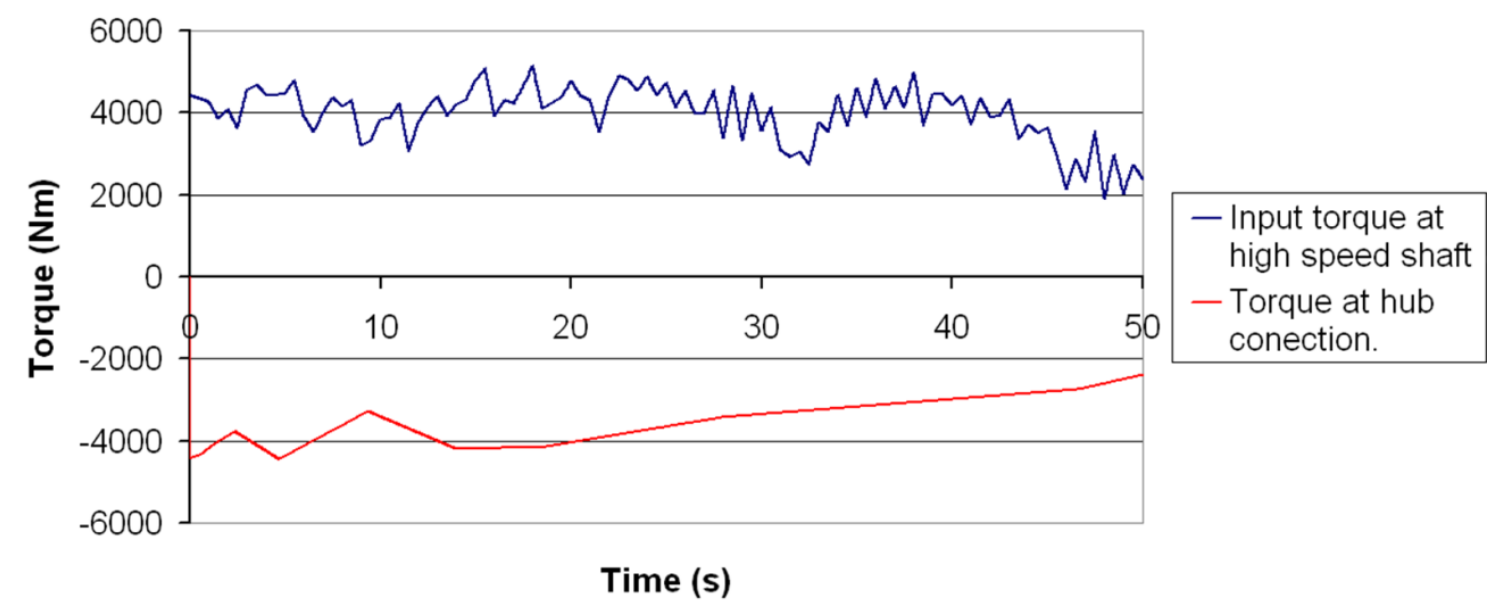

Figure 57. The input torque and output torque for the Stage 1 model

The same approach was used for the rest of the models, although a much closer response of the torsional behavior of the system was obtained. Figure 58 depicts the input torque and output torque of the gearbox. Due to the increment in torque gained from the gear ratio, two independent scales had to be used. Note that the response obtained more closely follows the characteristics of the input torque. Due to the similarity in the response of all models, however, only the Stage 3 model is shown. 


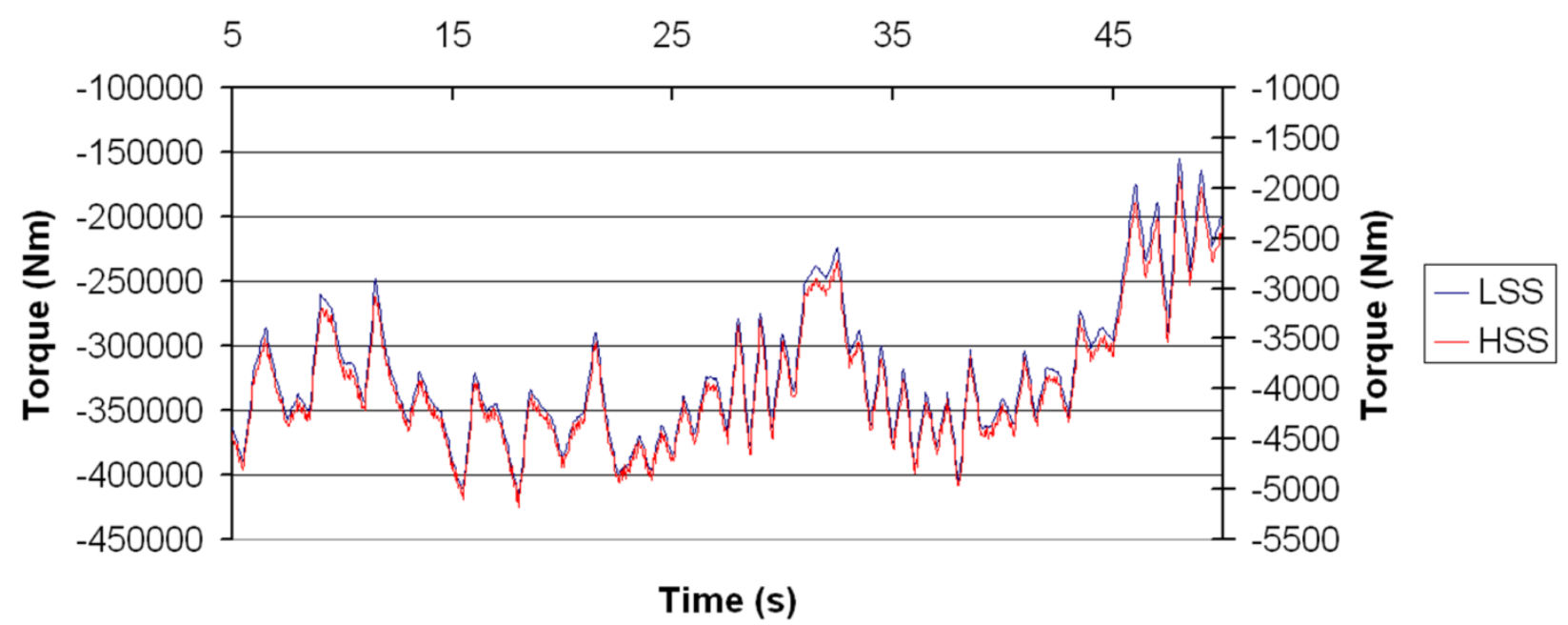

Figure 58. Input torque and output torque comparison for Stage 3

A comparison between the torque outputs of all models also was performed, and is shown in Figure 59. Note that the responses of the models are identical, and that torque for each is superimposed.

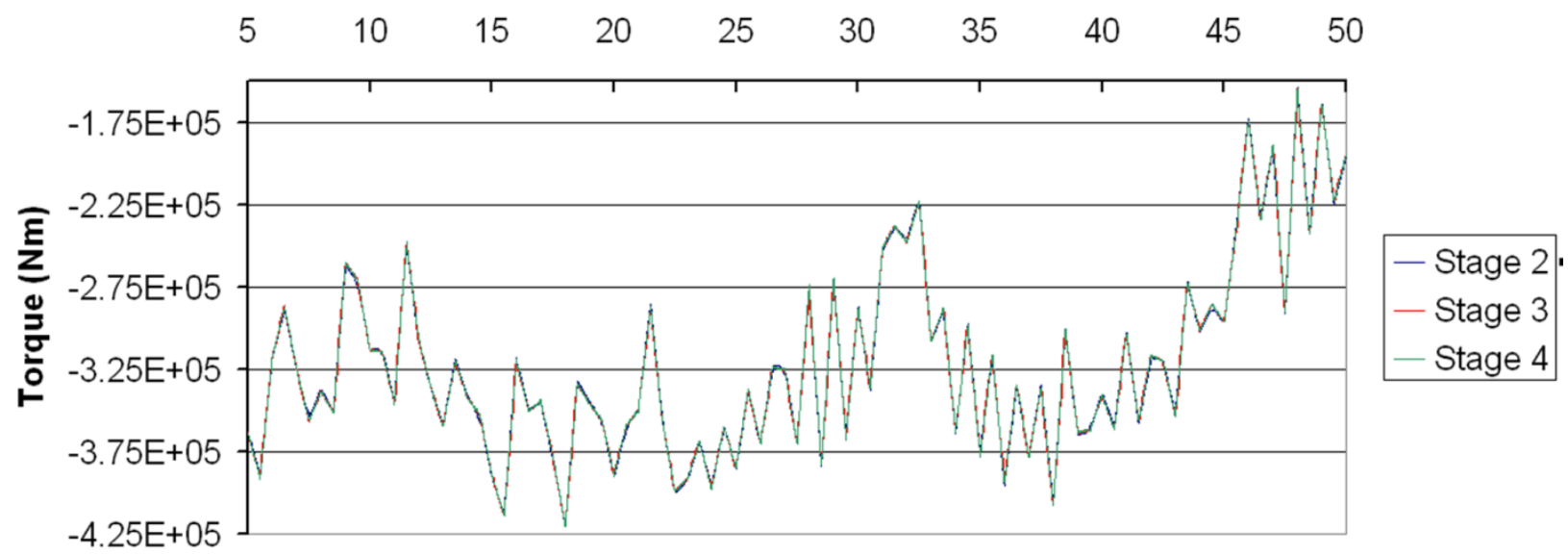

Time (s)

Figure 59. Stage 2 through Stage 4 torque comparisons

The intermediate shaft of the gearbox was chosen as a point of comparison between the stages because it is between the boundary conditions and it better captures the differences in the complexity of each stage. The parameter compared was the angular velocity of the intermediate shaft as taken at the gear. Figure 60 shows the recorded angular velocities of the simulation. The response obtained generally follows the same form, although it is noticeable that Stage 2 - which is the stage of least complexity in this comparison - has higher excitation than was expected at certain points of the simulation. 

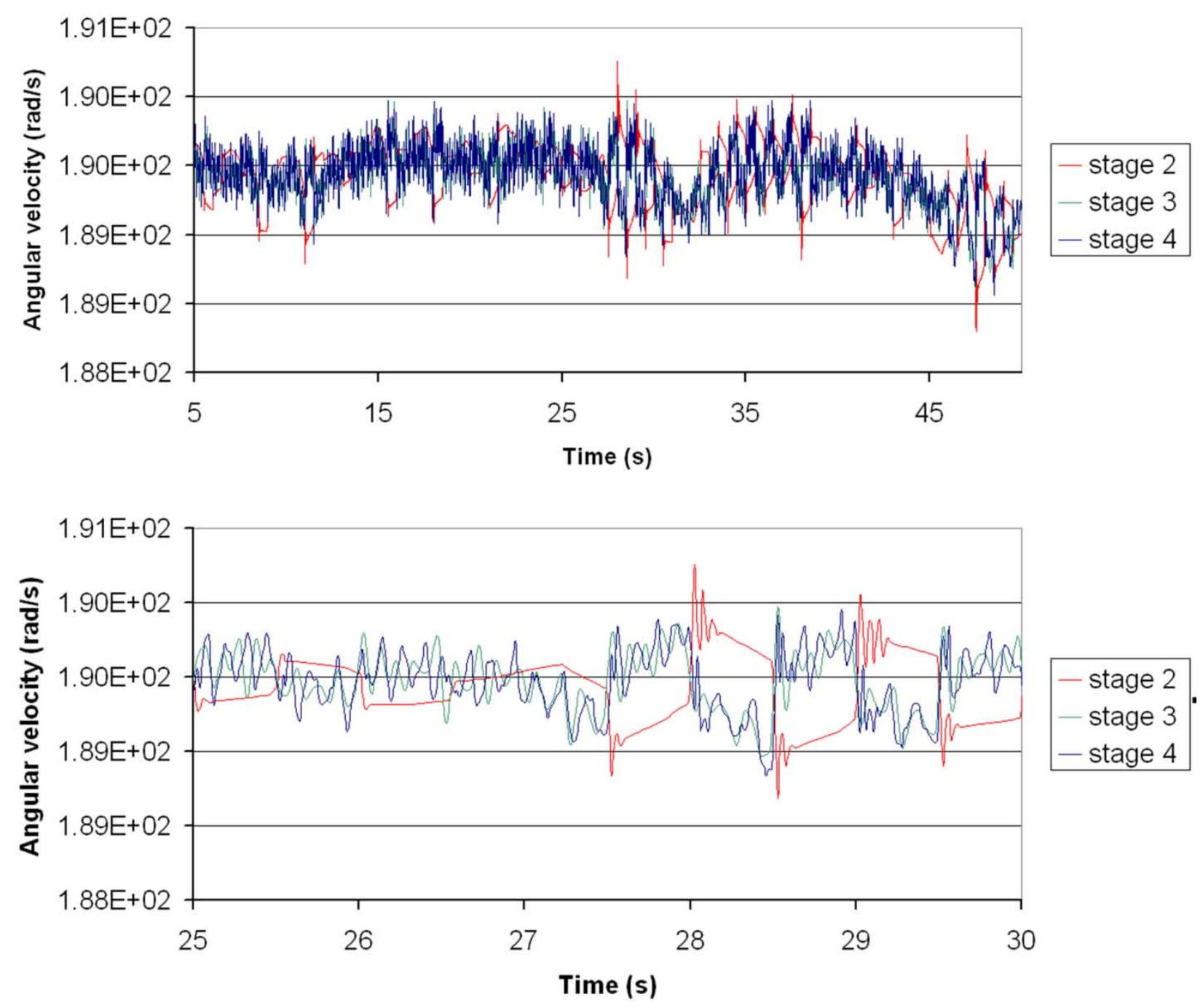

Figure 60. Stage 2 through Stage 4 angular velocity comparison

A closer examination of the same response reveals better insight into the importance of geartooth interaction. Note that the response obtained from Stage 2 is less complex than the responses from Stage 3 and Stage 4. The responses from Stage 3 and Stage 4 closely follow each other, although slight differences exist due to the additional degrees of freedom in Stage 4 .

Figure 61 compares the joint forces seen in the different stages. Stage 2 is not meant to include the loading of bearing, therefore its response is nearly zero. The results for Stage 3 and Stage 4 are quite similar, regardless of the single degree of freedom joint used in Stage 3. 


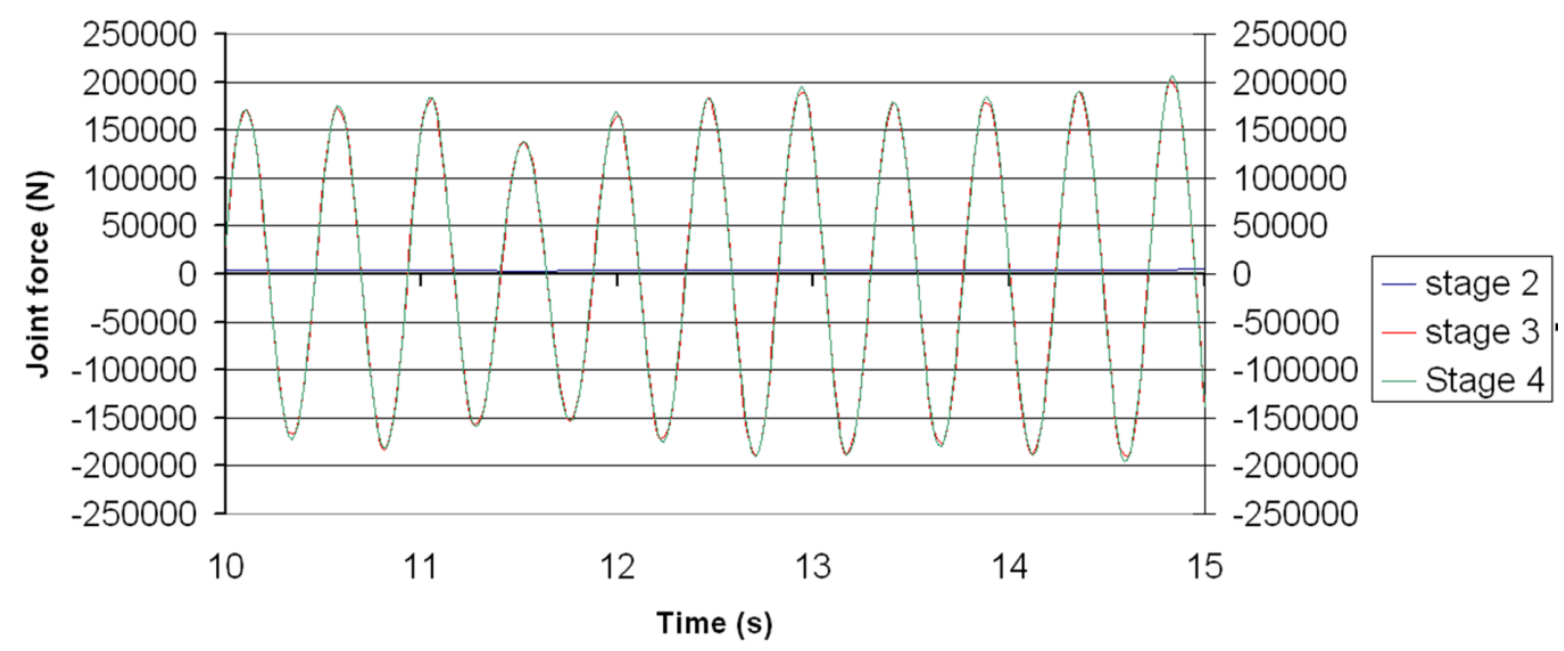

Figure 61. Joint force comparison of Stage 2 through Stage 4

Based on the previous comparisons, it seems that the model of Stage 1 is a good approach to perform a preliminary determination of stiffness that can be used to validate models of more complexity. This model, however, does not represent the actual behavior of the gearbox closely enough to be used for a more detailed analysis.

For Stage 2, the inclusion of all the components of the gearbox gives a much closer representation of its true behavior. This stage has an extremely close torsional response in comparison to the more complex models. Comparison of the angular velocity reveals that the behavior of the Stage 2 model follows the general behavior expressed by models of higher complexity. It already is noticeable, however, that the influences of gear-tooth contact are not accounted for in this model.

For Stage 3, the implementation of the gear-tooth elements provides a good representation of the behavior of the gearbox; depicting with better detail than Stage 2 the vibrational behavior and various excitations generated by the gear-tooth contact. This model has only one DOF per rotational body, therefore its computational time and stability are much better as compared to Stage 4. The implementation of only one degree of freedom per rotational body, however, results in the joined forces of the entire shaft being expressed as a single point. This quantity does not reveal the loading distribution among multiple bearings on a shaft. In the example presented the concurrence of both curves is very good, but with other configurations and the implementation of bearing clearances this situation might change. An important addition to the loads revealed by the Stage 3 model is the axial loading generated as a result of the helix angle in the gears. The comment made regarding load distribution among bearings also applies to this type of loading condition.

Stage 4 is the most complex model and therefore reveals the most information about the system. Although for many parameters it follows the response of Stage 3 quite closely, it additionally expands and reveals important information for the loading conditions of the bearings and gears. The most obvious of these additions is angular misalignment of shafts and other components. This stage shows the loading distribution among the different bearings of the gearbox, providing valuable insight into the loading conditions of particular bearings. 
Knowledge of the true loading conditions of the bearings, as well as the gears, potentially can enhance the understanding of the unexplained bearing failures currently seen in the industry. Additionally, models such as Stage 2 could be of great use for the design of other components of the drivetrain. Nevertheless, models such as Stage 4 should be implemented for the collaboration and iterative process between the gearbox manufacturer and the bearing supplier.

\section{Drivetrain Design Process}

The following sections explain the overall design process for the wind turbine drivetrain. Initial background research is introduced first followed by a description of the gearbox design process as a separate entity, and finally gearbox design is correlated to the design process in a description of the two main branches of standard drivetrain design process. The first branch describes a design process in which the wind turbine manufacturer owns the gearbox design; thus the design process occurs simultaneously and the iterative process of sharing information is almost transparent. The second design process describes the turbine manufacturer as a customer of the gearbox manufacturer, and so the gearbox manufacturer and the turbine manufacturer work as two separate entities that share the limited level of information necessary for the iterative process. This information briefly summarizes the experiences of Ed Hahlbeck P.E., Don McVittie, and Brian McNiff, who have extensive experience in the design process and the manufacture of drivetrains and gearboxes for the wind industry.

\section{Pre-Design Process}

Just as for any other industrial manufacturing process, the economical feasibility of a project is evaluated to see if the project can flourish. This typically is done using a cost of energy study. This study relies on a number of studies of existing machines that rate turbine size, head mass, and drivetrain configuration to their economical viability. To calculate the cash flow return, this study also considers the site where the turbines will be installed [26]. Cost of energy studies have revealed that there is a considerable range of viable turbines sizes; however, the construction of wind farms near populated areas has created the demand for quieter machines. Over time, this has driven the industry to design and build larger machines.

\section{Gearbox Design Process}

The initial stage of the gearbox design process (see Figure 62) defines the basic requirements that the gearbox must fulfill. Although the main requirement is the loads document, the outcome of this initial stage is the definition of the general configuration of the gearbox including: number of stages, epicyclic or helical, gear ratio, and general structure.

From this original configuration the gear design is carried through. This includes calculation of number of gear teeth and module and the center distances. Next come rating calculations, including the allowable surface and bending stresses, and safety factors. Shaft dimensions also are defined during this stage, which allows for the initial bearing selection that usually is obtained from a sizing catalogue. To corroborate the initial selection, this is followed by the cooperation of the bearing manufacturers that use in-house rating capabilities.

At this point a life analysis is performed on the selected gears. These fatigue calculations typically are based on the Miner Palmgren method, which calculates the cumulative fatigue damage due to the variable load spectrum. Common practice is to maintain constant ratios 
between pitch diameter and face width. If the desired life is not met, then dimensions are increased proportionally to meet the desired life requirements. This is closely followed by the introduction of micro-geometry to reduce local loading peaks due to elastic deformation of the bearings, shafts, and gear bodies.

Next the interfaces are satisfied. These typically include connections to the main shaft, as well as the high-speed shaft, and design and interaction of the torque arms with the bedplate. Several iterations could be required to satisfy the design objectives.

Aspects of the manufacturing process also must be discussed as part of the iterative process. This includes the final refinements of the design, material characteristics, and heat treating, in addition to outsourcing requirements for casting and forging. Final details such as oil cooling, sensors and data interfaces, corrosion protection, and noise also are considered.

The final part of the design process is the manufacturing of two prototype gearboxes. These are used for testing under specified loading conditions using the in-house dynamometer. The tested elements are dissected and the wear is assessed to identify and fine-tune any changes that must be made to the design before it is released for limited series " 0 " production and field testing in operating turbines at various test sites. After the completion of field testing, any modifications required by field-test results are implemented. This is followed by the release of the design for large-scale production and sale.

\section{Vertically Integrated Design Process Load Case Predictions}

After the overall layout of the drivetrain has been chosen, a number of parameters can be scaled from previous designs and existing turbines. These parameters, although imperfect, should closely represent the characteristics of the evolving design. The estimated parameters such as head mass, gear ratio, and gearbox stiffness are used in aeroelastic simulation codes such as FAST, ADAMS, and BLADED to create a rudimentary model. Using this model, a spectrum of load cases is simulated to obtain the baseline load document that initially guides the design process.

\section{Analysis and Iteration}

A more complete model of the drivetrain is developed from the loads document. The model describes most of the physical attributes of the drivetrain and includes 3-D modeling of all components. These models subsequently are meshed so that a finite element analysis can be performed. In this analysis, the load path through the rotor and independent components is observed. The analysis results in an iterative process that resizes the components until the allowable stresses are met.

\section{Gear Design}

The gears and internal components of the gearbox are designed simultaneously with the loadpath analysis. This process follows the AGMA 6006-A03 standard which dictates guidelines directly tailored for the design of wind turbine gearboxes. The standard specifies the use of the full-load spectrum and Miner's rule cumulative fatigue method, and mandates separate safety factors for pitting and bending. 


\section{Driving Load Cases}

Using the two previous analyses (in some design process practices) the most influential load cases or driving load cases are selected. The load cases are applied to the model and the results are compared with allowed yield stresses. If there is a small number of load cases strongly driving the design, then these are reviewed to determine whether they can be lessened. In other design process practices the full-load spectrum is used for the same calculations.

\section{Reiteration and Refinements}

Using the more accurate values for masses and stiffness, the loads document is updated and the remaining components are reiterated. After the iteration has converged to a satisfactory solution, the components of the gearbox are refined. Refinements include gear-tooth micro-geometry for noise reduction as well as bearing selection.

\section{Vibration Analysis}

A Campbell diagram should be created using the calculated masses and stiffness. This design predicts vibration and resonances inherent to the turbine. Mass and stiffness might have to be adjusted to keep resonances out of the operating range.

\section{Controls}

Based upon inertias, pitch, and yaw rates, alternative control systems can be integrated into the simulation. The controls will have an influence on the load cases and on the interaction of the overall system to extreme loads. The new loads will be put through the simulation process again, and perhaps be fine-tuned.

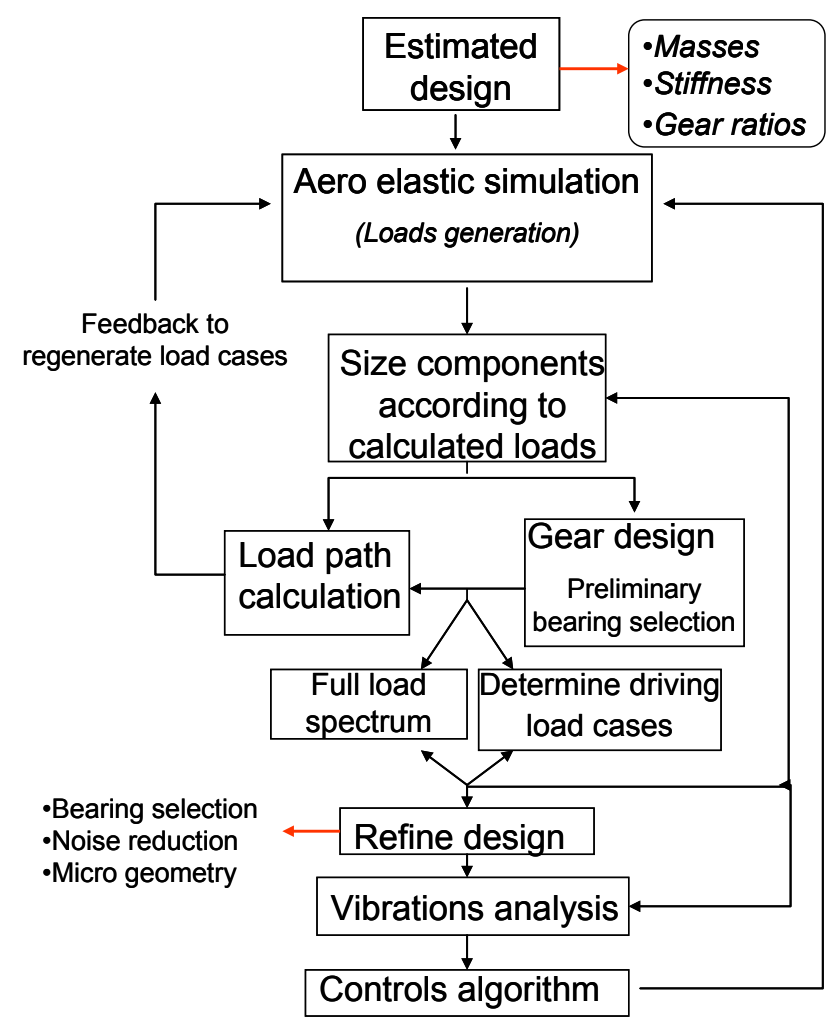

Figure 62. Fully integrated design process 


\section{Non-Vertically Integrated Design Process Develop Rotor and System Loads}

This section develops the rotor of the turbine in great detail (see also Figure 63). The remaining components of the turbine are estimated from previous designs. This information is sufficient for calculating (by means of aeroelastic simulation code) the first draft for a complete loads document.

\section{Drivetrain Definition}

At this point a definition of the drivetrain requirements is developed. This includes the gear ratio of the drivetrain as well as the requirements and specifications of the interfaces between the different components of the drivetrain. The reaction forces between interfaces also are defined.

\section{Drivetrain Specification}

Next, both previous steps are combined to create a document with the specification required for the gearbox manufacture. This document includes a load matrix describing time series for torsional and nontorsional loads and extreme load cases, and interface constraints and design parameters such as safety factor requirements and stress curve selection.

\section{Initial Design Review}

The drivetrain specification is given to potential gearbox providers who then develop an initial design. Each potential supplier is prequalified for design review. The review evaluates delivery and cost analysis as well as technical aspects of the design. The initial review narrows down the number of providers to be considered to go on with the project, or identifies specific provider.

\section{Design Selection}

A candidate's designs undergo the iterative process of satisfying the requirements for cumulative fatigue and the appropriate safety factors are applied. This is followed closely by a prototype design review in which the necessary details to fabricate a prototype are discussed and determined.

\section{Prototype and Testing}

The prototype and testing process typically starts with the fabrication of two gearboxes. The gearbox manufacturer performs a bench test with specified loads and a load duration matrix intended to simulate the lifecycle fatigue requirements. This is followed by a conditional certification of the gearbox. Certification allows the gearbox to be tested in the field -although this actually is more complicated because a number of newly designed components will be integrated, thus the complexity level of this test is much greater. The last step of the design process is the final prototype design review, which includes the disassembly of certain components of the turbine for evaluation. 


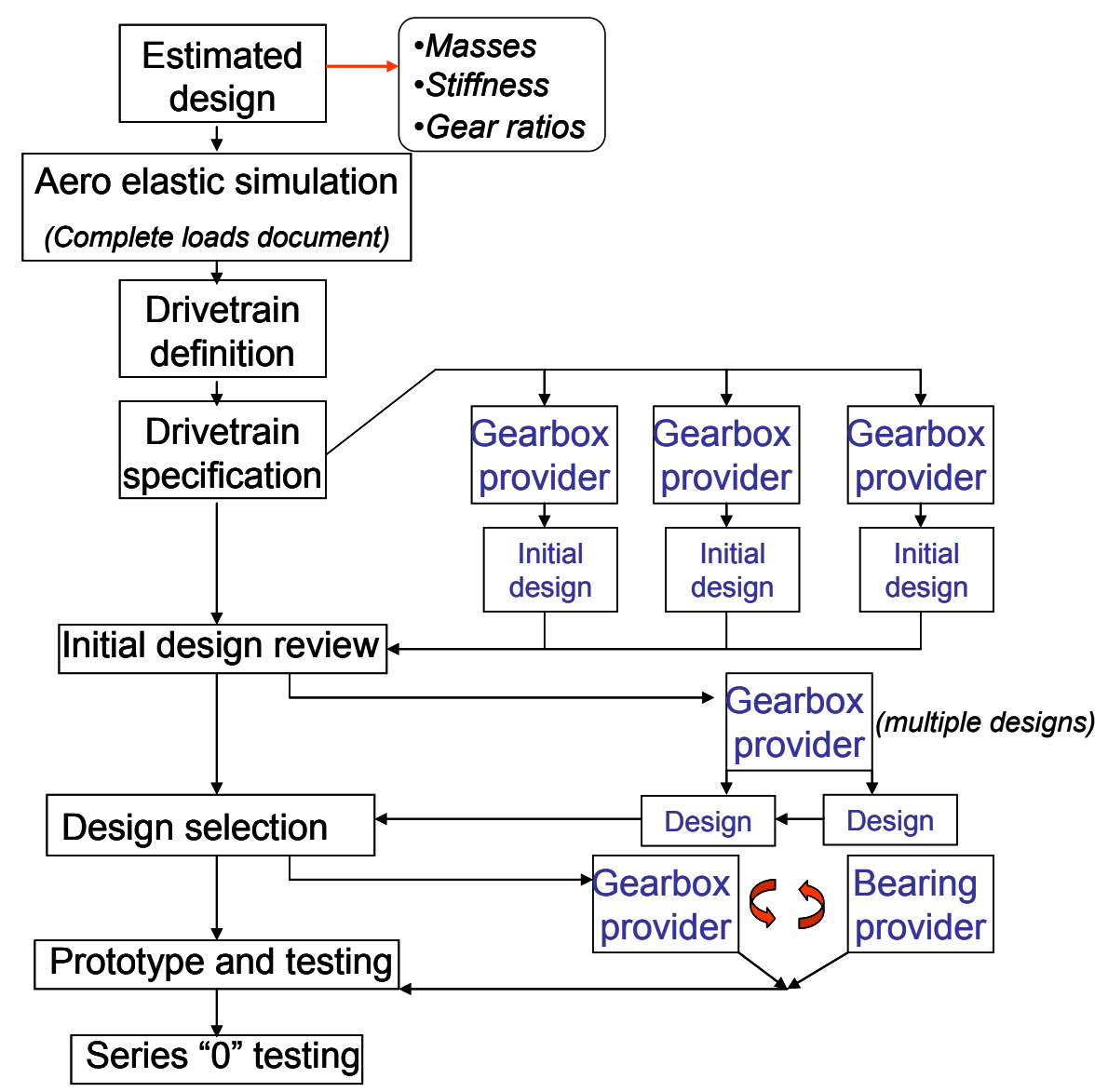

Figure 63 Non-vertically integrated design process

\section{Comparison of Drivetrain Design Processes}

The main difference between the vertically integrated design process and the non-vertically integrated drivetrain design process is the nature of the iterative process. The vertical design process not only iterates its individual subsystems (such as gearbox design), it also integrates the remaining components of the design into the overall iteration, creating a more complete process. This is achieved by a continuous update of the loading definition. All the different subsystems of the design depend on the loading conditions, therefore the process is valid throughout. This type of integration can be performed only when the wind turbine manufacturer internally manufactures the gearbox, or when the manufacturer has purchased the gearbox designs. This situation also can cause dependence on a specific provider or the need for the wind turbine manufacturer to fabricate gearboxes in house. Consequently, the most popular approach for drivetrain design is the decoupled design process.

As shown in Figure 60, the process is quite horizontal and does not include a great deal of iteration other than that of the independent subsystems. The overall calculations and ratings therefore are based on the initial assumptions for masses and stiffness, which define the load document. The reason for this situation stems from the interaction between the wind turbine manufacturers and the gearbox vendors involved in the process, rather than the definition or layout of the process itself. 
To be able to update the load document and integrate the iterative process into this design scheme, a great deal of information must be shared so that the wind turbine designer can create a comprehensive dynamic model. This design process involves a number of competing gearbox vendors, therefore the wind turbine manufacturer willingly shares only limited information. The gearbox vendors most likely also work for other wind turbine manufacturers, thus information from any particular wind turbine design is not shared. These informational barriers are the disengaging factors of the feedback process.

\section{Multistage Multibody System Simulation and the Design Process}

A detailed model of the gearbox components is needed to truly capture the dynamical behavior of the drivetrain and to properly predict the loading conditions of the system. The multistage approach to drivetrain design that is presented in this report enables the reinstatement of the iterative nature of the design process back into the non-vertical design process. The model's progressive nature can be used to "sanitize" the data shared among vendors and wind turbine manufacturers. This process could increase the level of useful modeling data that is shared, thus improving the level of accuracy of the overall model without increasing the sharing of sensitive and proprietary data.

The simple model used by the aeroelastic simulation codes commonly resembles the model in Stage 1, therefore the entire drivetrain is represented by a single degree of freedom. The advantage of this model is the simplicity of its input parameters, and that a minimal amount of data is given to the wind turbine manufacturer.

Stage 2-which already represents all moving components - provides a more accurate behavioral response of the system, which is required to calculate the loading conditions. Although this stage requires more gearbox geometry detail, the information given (general masses and gear ratios) is not of much value to competitors. Determining the stiffness coefficient required would be a trivial task for a gearbox manufacturer, because the manufacturer generally performs a complete finite-element analysis of the system.

Stage 3 requires a greater level of trust between parties. It requires providing the overall configuration of the gearbox, as well as the number of teeth and module. If the tooth geometry is sensitive information, then the geometry ratios described by the American Gear Manufacturers Association (AGMA) standard could be used. Regardless of the tooth geometry, the tooth microgeometry - which currently is a significant area of research for noise reduction and efficiencyis not shared.

Stage 4 involves the parameters noted for Stage 3 with the addition of the required bearing stiffnesses. Although the bearing stiffnesses do not have a great influence on the torsional behavior of the system, they are quite important for vibration analysis. In this case generalized stiffness data from the manufacturers are required. The stiffness response can be provided without revealing any of the geometrical characteristics of the bearing. The analysis methods used by the bearing manufacturer to calculate this stiffness are not revealed, which can protect the data integrity. Additionally, the present study did not seek to determine the sensitivity of a particular bearing stiffness, and a general stiffness for all bearings could be used thereby reducing the amount of data shared. 
The advantage of the progressive model approach is that it enables not only increasing complexity among models but also the possibility of a complexity reduction. The response of a more complicated model can be mimicked through implementation of complex stiffness parameters for a simpler model. For example, the Stage 3 model can be reduced to the Stage 2 model by implementing more complicated stiffness responses. Nonlinearities can be implemented to the stiffness and damping coefficients, and prescribed excitation can account for tooth interaction vibrations. This creates a model with a response that is closer to a more complicated model, even though all the sensitive parameters are hidden under the nonlinear stiffness. It is evident that the response of a system with fewer degrees of freedom would not be as accurate and would not mimic all the desired parameters. Nevertheless, using this approach allows creation of models that are closer to reality without necessitating the compromises generally involved; consequently, the loading parameters can be redefined to a more accurate level.

The detailed multibody system simulation (MBS) truly captures the dynamical behavior of all moving components. The approach, however, does not predict the minute mechanical and structural behavior, which necessitates the cooperation between different disciplines. For example, the creation of load cases for particular components of the drivetrain can be created from the MBS simulation. These load cases can be used in the finite element model, which can reveal in greater detail the mechanical behavior of the internal components such as tooth-load distribution and tooth-bending characteristics. The load conditions cover a wide spectrum of loading and load variations, which are narrowed to the load conditions of most relevance.

\section{Conclusions and Final Remarks}

Well-known standards - such as the AGMA standard 6006 and the Germanischer Lloyd certification - discuss the importance of the implementation of multibody system dynamics into the design process of wind turbine drivetrains. These guidelines do not specify the level of detail required for the fully coupled model, however, nor do they specify how these models can be integrated into the design process.

The premise of the research discussed in this report was to create a baseline model for a wind turbine drivetrain. Models were developed with increasing complexity and correlated in a progressive manner; they also were validated with experimental data collected from a representative wind turbine in the field. The progressive models created are representative of the gearbox and drivetrain standard of the industry, and include turbines that have output ranging from $750 \mathrm{~kW}$ to $5 \mathrm{MW}$.

Using FAST-AD software, an aeroelastic model also was created for the same representative field turbine. The aeroelastic model was used to create realistic load cases that could be input into the multibody system dynamic models. The results from the multibody system simulation from the input load cases were used to create load cases of the internal components of the gearbox that could be used for further, more-detailed analysis, which could be better integrated into the design process.

Different branches of the design process for the drivetrain of wind turbines also are discussed, including the fully integrated design and the coupled design. This report also examines the challenges in the data sharing between the wind turbine designers and the gearbox providers with 
respect to the design process. These barriers to information accessibility produced by the fierce competition and inherent proprietary data prevent the iterative nature of the design process.

This report suggests using the progressive multibody system dynamic approach as a data-sharing sanitization tool. Using a minimal amount of sensitive data, comprehensive and detailed models can be created to enhance the load-prediction methods of the design process, and return its iterative characteristics to the non-vertical design process.

The validation of the models using the obtained experimental data provides great insight into the correlation between theoretically calculated values and experimental empirical response. The theoretical data used in the various models had a disparity that was not greater than $10 \%$ for the stiffness coefficients and was not greater than $20 \%$ for the dampening coefficients. This finding is valuable because the theoretical values can be calculated using known physical dimensions of the gearbox; the response obtained will not differ greatly relative to the true response.

Comparison of the different models revealed that models having the level of complexity of Stage 1 should not be used for more than a rough estimation of the dynamical behavior. Stage 2 through Stage 4 gave concurrent responses for the torsional behavior of the different components of the system. In the case of angular velocities and acceleration, the models with greater complexity revealed a more complex loading response generated by the gear-tooth interactions. The Stage 4 model proved to be more valuable for the bearing- and gear-failure predictions, because it is capable of revealing misalignments of the different components of the gearbox.

It is worthwhile to note that the parameters used to validate the simulation were strictly linear. Thus, the simulated response does not exactly match the response obtained from the experimental data. The change in stiffness can be thought of as a change in frequency of the obtained response. The change in frequency is a consequence of clearances and component interactions that become more prominent as the magnitude of the excitation is reduced. Figure 64 shows the normalized change in the stiffness coefficient collected from the experimental data.

Models such as those for Stage 1 and Stage 2 do not depict the nonlinear behavior. In models such as those for Stage 3 and Stage 4, the effect of backlash has an influence on the overall stiffness of the system. This influence results in a change of frequency or stiffness that is similar to the change seen in the experimental response, although the correlation is not as strong. Additionally, the neglect of all the nontorsional flexibilities of the gearbox such as the shaft and housing, as well as the flexibilities of the bedplate, also could have influenced the misalignment and loading conditions of the drivetrain components. 


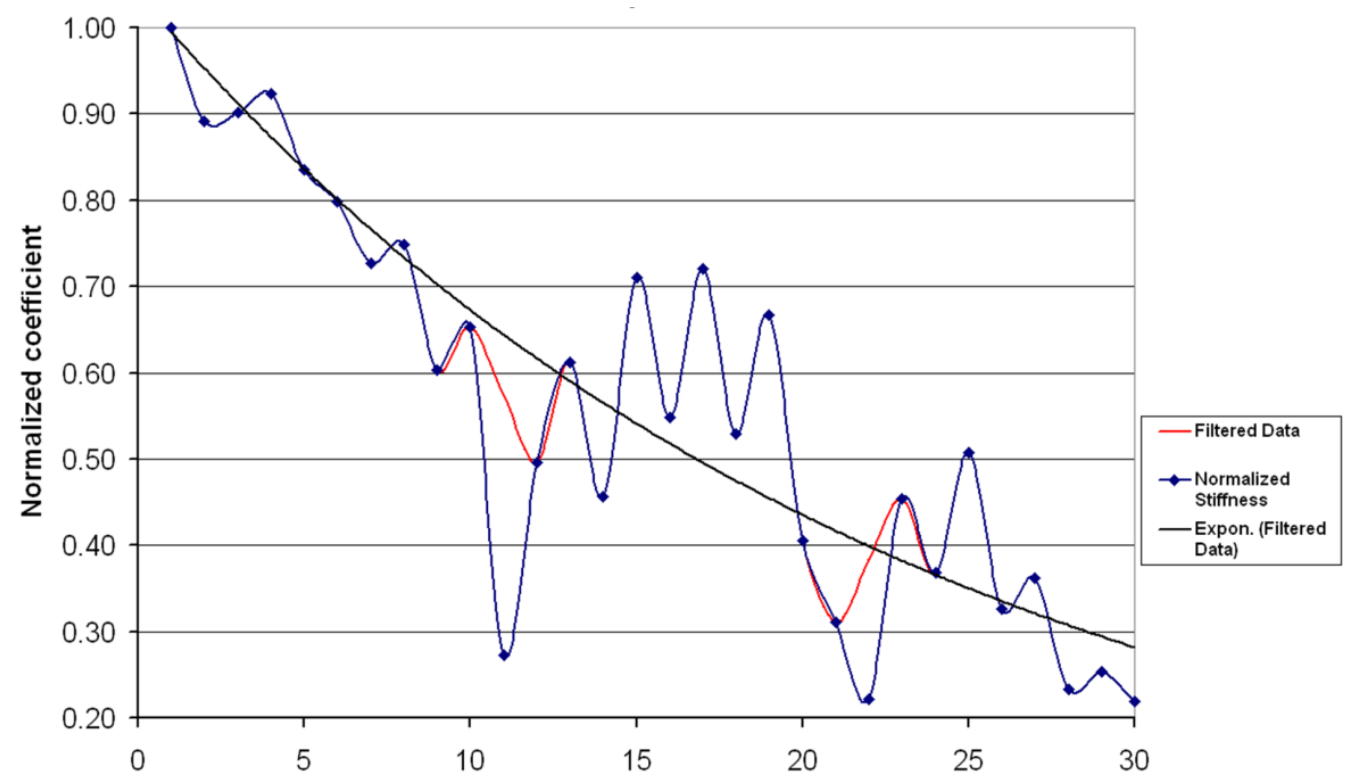

Figure 64. Stiffness coefficient decay from experimental data

\section{Future Work}

Further instrumentation and data collection will follow to better validate the current models. The data collected will include dynamometer as well as field data. The parameters collected from the dynamometer will include internal displacements, misalignments, and vibrations. The field data will include real operational inputs such as torque, and angular velocities of input and output. This information can be used to fine-tune the existing validated models, and to assure that the response simulated correlates with the true behavior of the machine.

The models currently built employ arbitrary stiffness and damping coefficients for the bearings. This approach reiterates the importance and capabilities of the MBS approach, although it does not reproduce the true behavior of the system. Further research revealing the nonlinear characteristics of the stiffness and damping coefficients of the bearings would be valuable. Research correlating bearing failure modes to specific load conditions also would be of great use, because particular loading conditions could be identified under simulated operational conditions.

The shafts in the Stage 4 model currently represented by rigid bodies will be substituted by flexible beam elements. These beam elements can handle nonlinearities of the system, and will allow eccentricities and misalignment of the gears in a more accurate manner. The shaft flexibilities can be obtained by a finite element analysis to find the modal behavior of the shaft and the modal stiffness. Performing a Guyan reduction reduces the number of degrees of freedom. This reduced element is imported into the multibody system simulation, enabling the system to represent the dynamical behavior and flexibilities [8]. Nevertheless, the Guyan reduction keeps the computation light, thus allowing for simulation of longer events. The same modal approach can be implemented for gearbox housing, simulating the flexibilities of the housing under dynamical loading. The deformation resulting from this flexibility also can contribute to gear misalignment and internal compliances of the system. 
Further analysis will follow the dynamical analysis, including finite element analysis of the gearbox, and the implementation of the generated dynamical input load cases. This detailed model will reveal important factors such as tooth load distribution, which can be correlated to fatigue and other failure modes. To truly capture the overall dynamical coupled behavior of the system the dynamical model should be extended to include other components of the drivetrain, such as generator and rotor. 


\section{References}

1. Wind Turbine Data Summary Table 2. Wind Stats Newsletter. Volume 16 - 19, Numbers 1 - 4, 2003 - 2006. Volume 20, Number 1, 2007.

2. Walford, C. (2006). O\&M Cost Model Quantifying the Influrnce of Reliability, Wind Turbine Reliability Workshop. Global Energy Concepts Seattle, WA. cwalford@globalenergyconcepts.com http://www.sandia.gov/wind/2006reliability/wednesday/09-chriswalford\%20.pdf

3. Gasch, R.; Twele, J. (2002). Wind Power Plants Fundamentals: Design, Construction and Operation. Berlin: Solarpraxis AG.

4. Poore R.; Lettenmaier, T. (2002). Alternative Design Study Report: WindPAC. Kirkland Washington: Advanced Wind Turbine Drivetrain Design Study Global Energy Concepts, LLC.

5. Manwell, J. F.; Mc Gowan, J. G.; Rogers, A. L. (2002). Wind Energy Explained: Theory, Design and Application. Chichester, NY: Wiley.

6. Norton, R. L. (2006). Machine Design: An Integrated Approach, 3rd edition. Upper Saddle River, NJ: Pearson Prentice Hall.

7. Ackerman, T. (2005). Wind Power in Power Systems. Hoboken, NJ: John Wiley.

8. Drago, R. J. (1988). Fundamentals of Gear Design. Boston, MA: Butterworths.

9. Spotts, M. F. (1951). Design of Machine Elements, 4th edition. London: G. Allen \& Unwin.

10. ANSI/AGMA (1980). AGMA Standard 110.04. Nomenclature of Tooth Failure Modes (reaffirmed 1989).

11. Jonkman, J. M. (2003). Modeling of the UAE Wind Turbine for Refinement of FAST_AD. NREL TP-500-34755. Golden, CO: National Renewable Energy Laboratory.

12. Popov, E. P.; Balan, T. A. (1998). Engineering Mechanics of Solids, 2nd edition. Upper Saddle River, NJ: Prentice Hall.

13. Angeles, J. (ed.) (1995). Kinematics and Dynamics of Multi-Body Systems. Wien, NY: McGill University and A. Kecskemethy Gehard Mercator University.

14. INTEC GmbH. SIMPACK Basics Training 1. Wessling, Germany.

15. INTEC GmbH. SIMPACK User Manual. Force Element Library. Wessling, Germany.

16. Ramamurti, V. (2000). Mechanical Vibration Practice with Basic Theory. Boca Raton, FL: CRC Press/Narosa Publishing House.

17. Palm, W. J. III (2007). Mechanical Vibration. Hoboken, NJ: John Wiley.

18. Jonkman, J. NWTC Design Codes, FAST, An Aeroelastic Design Code for Horizontal Axis Wind Turbines. http://wind.nrel.gov/designcodes/simulators/fast (accessed Sept. 18, 2008).

19. Peeters, J. L. M.; Vandepitte, D.; and Sas, P. (2005). “Analysis of Internal Drive Train Dynamics in a Wind Turbine." Wind Energy, 9(1-2), pp. 141-161.

20. Rivin, E. I. (1999). Stiffness and Damping in Mechanical Design. New York: Marcel Dekker.

21. INTEC GmbH. SIMPACK User Documentation. III-FE:14 Gearbox with Elastic Transmission. Wessling, Germany.

22. INTEC GmbH. SIMPACK User Documentation. III-FE:225 Gear Pair. Wessling, Germany.

23. INTEC GmbH. SIMPACK FEMS Training Course. Wessling, Germany.

24. INTEC GmbH. SIMPACK Basics Training Course. Wessling, Germany. 
25. Gipe, P. (1993). Wind Power: Renewable Energy for Home, Farm, and Business. Post Mills, VT: Chelsea Green Publishing Co.

26. National Wind Technology Center (2006). Baseline Cost of Energy. http://www.nrel.gov/wind/coe.html (accessed Sept. 18, 2008).

27. NEG Micon A/S (2000). Weights, Dimensions and Transport Guidelines NM 750/48. TIC247’002 GB, Provided by Turbine operator Ken Bolin Excel Energy.

28. Gold, P. W.; Schelenz, R.; Frenschek, W.; Klein, A.; Moller, D. (2004). Simulation of the Three-Dimensional Vibration Behavior of a Wind Energy Plant. SIMPACK User Meeting, 2004. Available at http://www.simpack.com/downloads/pdf/ um04_rwth moeller.pdf (accessed Sept. 18, 2008).

29. Moriarty, P. J.; Hansen A. C. (2005). AeroDyn Theory Manual. NREL/TP-500536881. Golden, CO: National Renewable Energy Laboratory.

30. Jonkman, J. M. (2003). Modeling of the UAE Wind Turbine for Refinement of FAST_AD. NREL/TP-500-34755. Golden, CO: National Renewable Energy Laboratory.

31. ANSI/AGMA. (2004). AGMA Standard 2001-D04. Fundamental Rating Factors and Calculation Methods for Involute Spur and Helical Gear Teeth.

32. Heege, A. (2003). "Computation of Dynamic Loads in Wind Turbine Power Trains." DEWI Magazine 23, pp. 59-64. Available at http://www.dewi.de/dewi/fileadmin/pdf/ publications/Magazin_23/08.pdf (accessed Sept. 18, 2008).

33. Wilcoxon Research. Bearing Failures: Causes and Cures. http://www.wilcoxon.com/ knowdesk/bearing.pdf (accessed Sept. 18, 2008).

34. DeLange, G. Failure Analysis for Gearing. http://www.elecon.com/gearworld/dat-gwfailure.html (accessed Sept. 18, 2008).

35. Schaeffler Group Industry (2006). International Standard ISO 1524. Ad-hoc Meeting Orlando, Florida.

36. Geartech (1999). Gear Failure Atlas. Townsend, MT: Geartech.

37. GEEA. Nordex diagram. http://www.geea.org/IMG/jpg/vue_eolienne_nordex1000.jpg (accessed Sept. 18, 2008).

38. Mauer, L. (2006). The New, Powerful Gearwheel Module. SIMPACK User Meeting 2006 INTEC GmbH, Wessling, Germany. Available at http://www.simpack.com/downloads/ pdf/um06_intec_mauer.pdf (accessed Sept. 18, 2008). 


\section{Appendix: Aerodynamic Simulation}

\section{One-Dimensional Momentum Theory}

One-dimensional momentum theory is known as the Betz momentum theory, and describes the behavior of an ideal wind turbine rotor. This simple model takes into account only the axial downstream losses. The theory assumes a tubular control volume which starts far away from the rotor and ends far behind the rotor. Inside of the control volume the rotor is represented by an actuator disc. As the inflow passes through the actuating disk a discontinuity in pressure is created (Figure 65, V1). The pressure of the control volume remains constant therefore the crosssectional area increases (Figure 65, V2), resulting in the reduction of the flow velocity behind the rotor (Figure 65, V3)

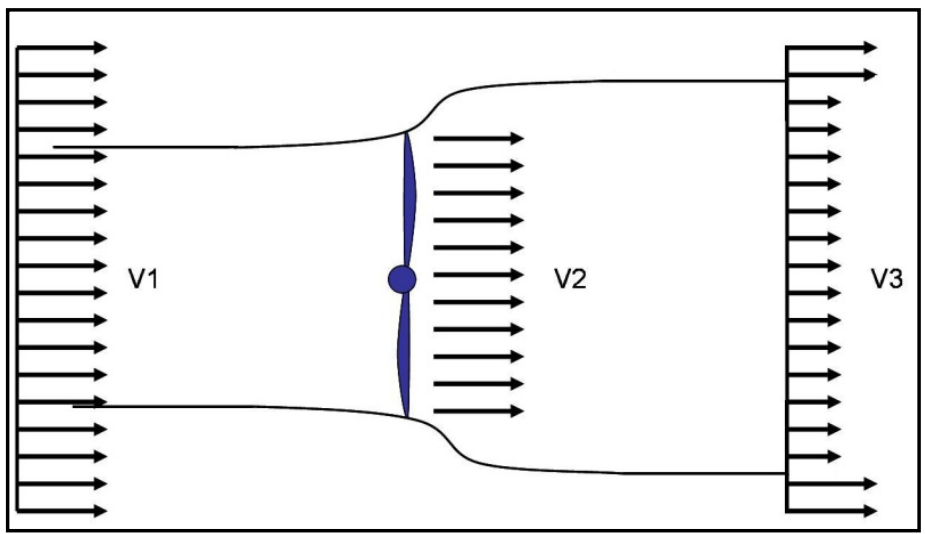

Figure 65. Betz controlled volume with respective velocities [3]

The thrust of the turbine can be calculated using the principle of linear momentum, because the thrust is equal in magnitude and opposite in direction to the change in momentum [5]. As the control volume increases in area and the outgoing velocity reduces, the mass flow remains constant. Thus, the change in angular momentum is solely dependent on the change in flow velocity. The thrust can be calculated from the following expression.

$$
T_{r o t}=\dot{m}\left(V_{1}-V_{3}\right)
$$

If there is no reduction in the flow velocity and the flow passes without resistance, then there is no change in momentum and no thrust or power is generated. Furthermore, if the flow is stopped completely then the mass flow rate is zero; consequently, the change in momentum is zero as well. This results in an optimum velocity ratio of $1: 3$ between the outgoing velocity (V3) at the end of the control volume and the ingoing velocity (V1) entering the control volume. This allows the calculation of the power coefficient, as well as giving a max power of $\mathrm{Cp}=0.59$, which is the power coefficient for an ideal turbine. [3]

\section{Ideal Wind Turbine with Wake Rotation}

The model described above can be extended to account for the rotational wake shed by the turbine rotor (see Figure 66). This response can be attributed to Newton's third law, which states 
that for every action there is an equal and opposite reaction. Consequently, an angular momentum that rotates in a direction opposing the rotor is induced on the wake.

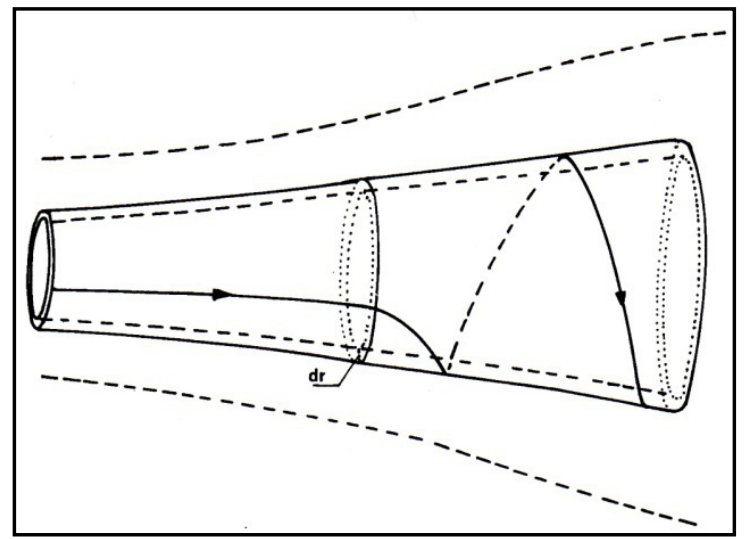

Figure 66. Controlled volume with rotational wake [5]

As was done in the previous analysis, the principle of conservation of momentum is used to calculate the corresponding load. The main difference is that the control volume is subdivided into a number of annuli to better account for the change in rotation of the wake. In this case, the expression for torque experienced on the rotor can be derived using conservation of angular momentum.

\section{Blade Element Theory}

Blade element theory is a technique used to calculate the forces exerted on a wind turbine, such as thrust and torque. These forces are calculated from the interaction of the flow stream and the rotor. The calculations can be performed using the geometrical characteristics of the rotor, such as airfoil, shape, and twist distribution.

To better understand the simulation theory, two important quantities should be defined. The first is the lift force, which is the driving force of any lift devise. Lift force can be defined as the mechanical force generated as a solid travels through a fluid; it is perpendicular to the inflow of the solid. More specifically, lift is generated as a fluid travels over the airfoil by creating lower pressure above the profile. This occurs due to the convex surface of the profile increasing the flow velocity and inherently reducing the pressure. The concave side or bottom side, in contrast, generates greater pressure. These low and high pressure differences result in lift force - the low pressure pulls the profile and the high pressure pushes the profile [30]. The other important quantity is the drag force. Drag force is a force which opposes the motion of the airfoil as it travels through the fluid, and most applications seek to minimize this force. Drag force is generated by the viscous effects of fluid traveling over the profile and by unequal pressure distribution on the airfoil [30]. These two measures result in a friction force that is parallel to the inflow [5].

Lift and drag can be characterized as dimensionless parameters called lift and drag coefficients. The values of these coefficients greatly depend on the airfoil profile and angle of attack, and are less dependent on the size and the relative speed of the flow. Coefficient of lift, $C_{l}$, and coefficient of drag, $C_{d}$, can be calculated using the following expressions. [11] 


$$
C_{l}=\frac{L / l_{s}}{\frac{1}{2} \rho U^{2} c}
$$

$$
C_{d}=\frac{D / l_{s}}{\frac{1}{2} \rho U^{2} c}
$$

Here, $L$ is the lift force, $D$ is the drag force, and $l_{s}$ is the airfoil span. In the denominator, the dynamic forces per unit length are taken into account; $\rho$ is the fluid density, $U$ is the velocity of the undisturbed fluid flow, and $c$ is the cord length of the airfoil. [5]

These parameters generally are determined experimentally in a wind tunnel [3], where scaleddown airfoils are tested under controlled flow conditions. The lift and drag forces are recorded at a range of angles of attack and the coefficients are calculated (see Figure 67).

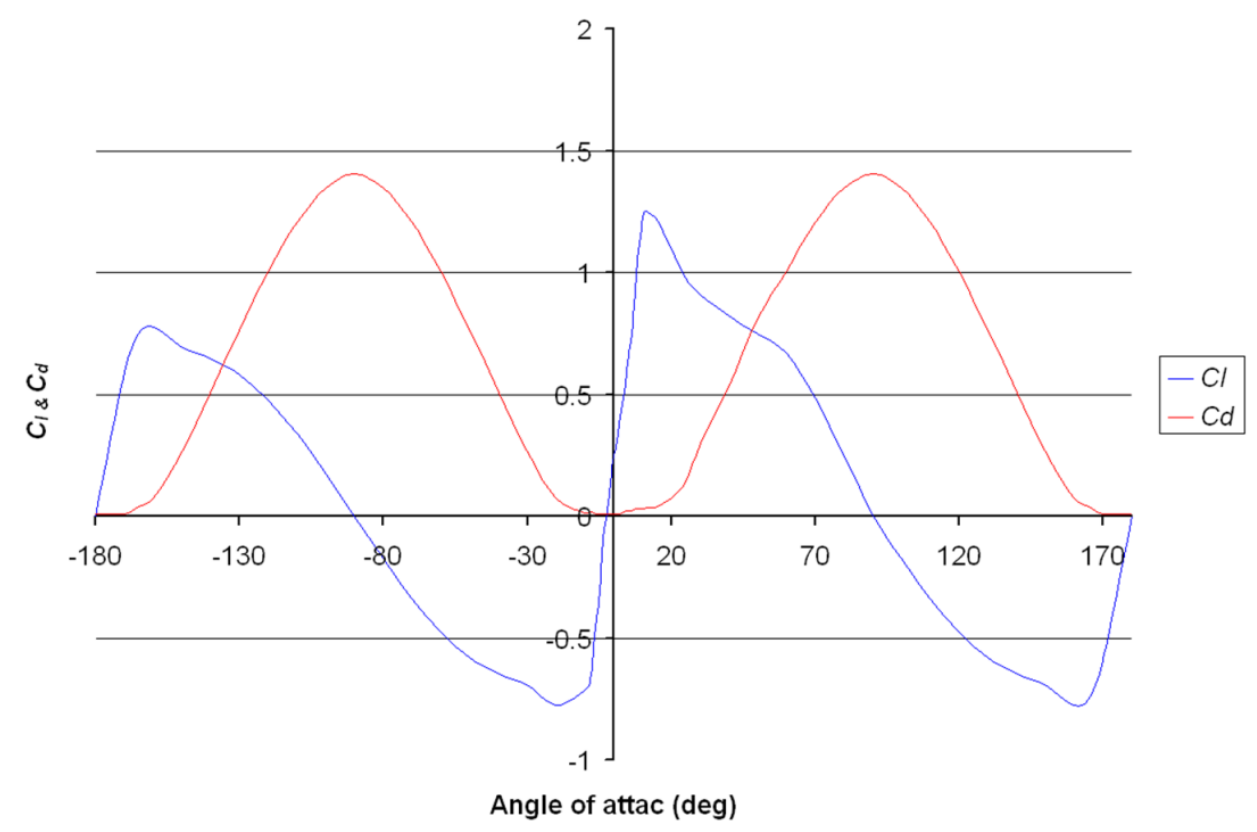

Figure 67. Curves for coefficient of lift and coefficient of drag

At lesser angles of attack the lift coefficient increases linearly, with the angle of attack increasing to approximately $10^{\circ}$. The curve then tends to flatten before reaching its maximum value [3]. This is the result of the initial separation of the flow above the airfoil. As the angle of attack increases the separation becomes more evident, and a drastic reduction of the lift and increase in the drag can be seen. At greater angles of attack the behavior of the airfoil resembles a flat plate. Wind turbines operate in this range, therefore the behavior is estimated using this assumption for the entire $360^{\circ}$ range [5].

The blade element theory subdivides the blade along its length into a number of elements. These elements sweep a number of annuli as the rotor rotates. The theory assumes that there is no interaction between each blade element, and that the behavior of the blade profile follows the two-dimensional lift and drag characteristics of the profile [11]. The lift and drag forces therefore can be calculated with the previously described lift and drag coefficients. 
The missing quantity is the angle of attack for each individual section of the blade. The angle of attack is dependent on the inflow angle and the particular angle of twist of the blade. The inflow angle is the result of a vectorial addition involving the tangential velocity of the blade element and the incoming wind. The tangential velocity of the particular section of blade can be calculated from the angular velocity of the rotor and the radial distance of the element with respect to the axis of rotation. The calculated lift and drag forces must be decomposed into perpendicular and parallel components with respect to the rotor sweeping plane. This decomposition results in the thrust for the perpendicular force and the torque for the tangential force. This procedure must be repeated for each individual element along the blade, and the overall thrust and torque of the rotor are the sum of all the element forces composing the rotor [29]. 


\section{REPORT DOCUMENTATION PAGE}

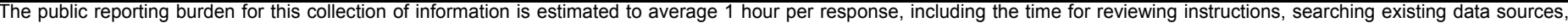

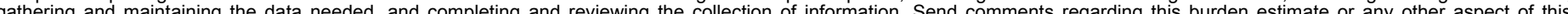
(0704-0188). Respondents

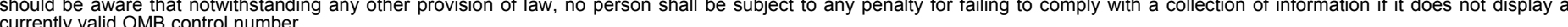

PLEASE DO NOT RETURN YOUR FORM TO THE ABOVE ORGANIZATION.

\begin{tabular}{l|l|l|l} 
1. REPORT DATE $(D D-M M-Y Y Y Y)$ & 2. REPORT TYPE & 3. DATES COVERED (FrOm - TO)
\end{tabular}

February 2009

4. TITLE AND SUBTITLE

Gearbox Modeling and Load Simulation of a Baseline 750-kW Wind

Turbine Using State-of-the-Art Simulation Codes

5a. CONTRACT NUMBER

DE-AC36-08-GO28308

5b. GRANT NUMBER

5c. PROGRAM ELEMENT NUMBER

6. AUTHOR(S)

F. Oyague

5d. PROJECT NUMBER

NREL/TP-500-41160

5e. TASK NUMBER

WER8.2001

5f. WORK UNIT NUMBER
7. PERFORMING ORGANIZATION NAME(S) AND ADDRESS(ES)

National Renewable Energy Laboratory

1617 Cole Blvd.

Golden, CO 80401-3393

9. SPONSORING/MONITORING AGENCY NAME(S) AND ADDRESS(ES)

\section{PERFORMING ORGANIZATION REPORT NUMBER \\ NREL/TP-500-41160} NREL

11. SPONSORING/MONITORING AGENCY REPORT NUMBER

12. DISTRIBUTION AVAILABILITY STATEMENT

National Technical Information Service

U.S. Department of Commerce

5285 Port Royal Road

Springfield, VA 22161

13. SUPPLEMENTARY NOTES

14. ABSTRACT (Maximum 200 Words)

This report discusses the causes for premature wind turbine gearbox failure and determining a method for revealing the missing loading conditions relevant to the gearbox design process.

15. SUBJECT TERMS

wind turbine design models; gearbox failures; wind turbine design analysis

\begin{tabular}{|c|c|c|}
\hline $\begin{array}{l}\text { a. REPORT } \\
\text { Unclassified }\end{array}$ & $\begin{array}{l}\text { b. ABSTRACT } \\
\text { Unclassified }\end{array}$ & $\begin{array}{l}\text { c. THIS PAGE } \\
\text { Unclassified }\end{array}$ \\
\hline
\end{tabular}

\begin{tabular}{l|l} 
17. LIMITATION & 18. \\
OF ABSTRACT & OF PAGES \\
UL &
\end{tabular}

19a. NAME OF RESPONSIBLE PERSON

19b. TELEPHONE NUMBER (Include area code) 